\title{
Measurement of the top quark mass using events with a single reconstructed top quark in pp collisions at
}

$$
\sqrt{s}=13 \mathrm{TeV}
$$

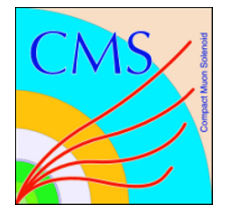

\section{The CMS collaboration}

E-mail: cms-publication-committee-chair@cern.ch

ABSTRACT: A measurement of the top quark mass is performed using a data sample enriched with single top quark events produced in the $t$ channel. The study is based on protonproton collision data, corresponding to an integrated luminosity of $35.9 \mathrm{fb}^{-1}$, recorded at $\sqrt{s}=13 \mathrm{TeV}$ by the CMS experiment at the LHC in 2016. Candidate events are selected by requiring an isolated high-momentum lepton (muon or electron) and exactly two jets, of which one is identified as originating from a bottom quark. Multivariate discriminants are designed to separate the signal from the background. Optimized thresholds are placed on the discriminant outputs to obtain an event sample with high signal purity. The top quark mass is found to be $172.13_{-0.77}^{+0.76} \mathrm{GeV}$, where the uncertainty includes both the statistical and systematic components, reaching sub-GeV precision for the first time in this event topology. The masses of the top quark and antiquark are also determined separately using the lepton charge in the final state, from which the mass ratio and difference are determined to be $0.9952_{-0.0104}^{+0.0079}$ and $0.83_{-1.35}^{+1.79} \mathrm{GeV}$, respectively. The results are consistent with $C P T$ invariance.

KEYwords: Hadron-Hadron scattering (experiments), Top physics

ARXIV EPRINT: 2108.10407 


\section{Contents}

1 Introduction 1

2 The CMS detector 3

3 Simulation of events 4

4 Event reconstruction 5

4.1 Event selection 5

4.2 Event categories 7

4.3 Reconstruction of the top quark 7

5 Estimation of the QCD multijet background 8

$\begin{array}{llr}6 & \text { Multivariate analysis } & 9\end{array}$

$\begin{array}{lll}7 & \text { Extraction of the top quark mass } & 13\end{array}$

8 Systematic uncertainties $\quad 16$

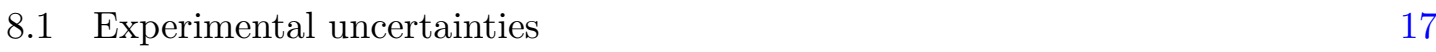

$\begin{array}{ll}8.2 \text { Modeling uncertainties } & 18\end{array}$

9 Results 21

10 Summary $\quad 25$

$\begin{array}{ll}\text { A MVA input variables and their correlations } & 26\end{array}$

B Likelihood scan and correlations among fit parameters 30

$\begin{array}{ll}\text { The CMS collaboration } & 36\end{array}$

\section{Introduction}

The mass of the top quark, $m_{\mathrm{t}}$, is an important parameter of the standard model (SM) of particle physics. Its precise measurement is of profound importance, both for theory and experiment. On the theory side, it constitutes a major input to global electroweak (EW) fits [1, 2], used to verify the self-consistency of the SM. It is also directly related to the stability of the EW vacuum, because among all known elementary particles it has the largest contribution in terms of radiative corrections to the Higgs boson mass $[3,4]$. From the experimental perspective, it also provides a benchmark for the identification and 

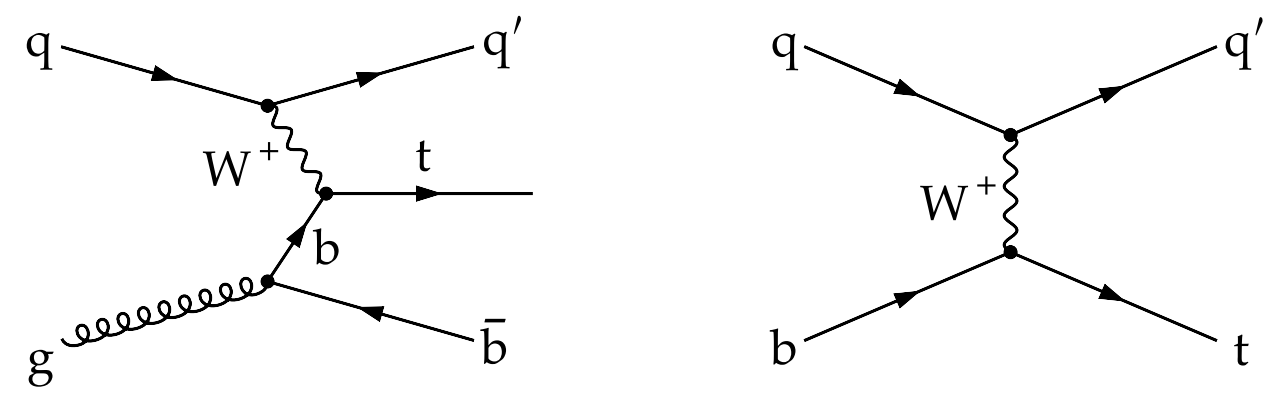

Figure 1. Feynman diagrams of the $t$-channel single top quark production at LO corresponding to four- (left) and five-flavor (right) schemes. At NLO in perturbative QCD, the left diagram is also part of the five-flavor scheme.

calibration of heavy-flavor jets [5] arising from bottom or charm quarks, both in resolved and boosted topologies [6].

The majority of LHC results on $m_{\mathrm{t}}[7-14]$ have been obtained with top quark pair $(\mathrm{t} \overline{\mathrm{t}})$ events. Such events are predominantly produced via gluon-gluon fusion (90\%) along with a subdominant contribution from quark-antiquark annihilation (10\%). The most precise $m_{\mathrm{t}}$ measurements of $172.08 \pm 0.48$ and $172.44 \pm 0.49 \mathrm{GeV}$ are reported by the ATLAS and CMS experiments, respectively, in $t \bar{t}$ events by combining results based on data recorded at $\sqrt{s}=7$ and $8 \mathrm{TeV}[7,8]$. Current models for color reconnection (CR) $[15,16]$ indicate that it can have a potentially large impact on the measured $m_{\mathrm{t}}$ values $[9,10]$. Measurements based on complementary event topologies are important to better understand such systematic effects, as well as to possibly reduce the impact of dominant sources of uncertainty via a combination of results.

Top quarks can be singly produced at the LHC through charged-current EW interactions via the exchange of a $\mathrm{W}$ boson. At leading order (LO) in the SM, single top quark production can be realized in three modes, depending on the virtuality of the $\mathrm{W}$ boson involved in the process, namely the $t$ channel (spacelike), the tW channel (on-shell), and the $s$ channel (timelike). The $t$-channel diagram shown in figure 1 constitutes the dominant process for single top quark production in proton-proton (pp) collisions at the LHC, with a total cross section of $217_{-8}^{+9} \mathrm{pb}$ at $\sqrt{s}=13 \mathrm{TeV}$ calculated at next-to-LO (NLO) $[17,18]$ in perturbative quantum chromodynamics (QCD). Within uncertainties, the measured cross section [19] agrees with this prediction.

The $t$-channel single top quark production occurs at a lower energy scale $(\geq 170 \mathrm{GeV})$ compared to $\bar{t} \overline{\mathrm{t}}(\geq 340 \mathrm{GeV})$, offering a partially independent event sample for measurement in a complementary region of phase space. Furthermore, it enhances the range of available measurements with systematic sources that are partially uncorrelated from those for $t \bar{t}$ events. The $t$-channel process involves CR only between the top quark and the proton from which the b quark or the gluon in the initial state arises, not to the whole event, unlike in the case of $t \bar{t}[16]$. Such alternative measurements provide an important validation for the modeling of nonperturbative QCD processes in Monte Carlo (MC) simulations, which could be a source of large systematic effects. The unique production mode and event topology 
may also shed light on the interpretation of the $m_{\mathrm{t}}$ parameter [20], since, as opposed to t $\overline{\mathrm{t}}$ events, the single top quark process probes energy scales down to the production threshold of about $170 \mathrm{GeV}$.

The signature of $t$-channel single top quark production comprises an isolated highmomentum charged lepton, a neutrino, which results in an overall transverse momentum $p_{\mathrm{T}}$ imbalance, a light-quark jet, often produced in the forward direction, and a jet arising from the hadronization of a bottom quark (b jet) from the top quark decay. The second $\mathrm{b}$ jet, arising in the production process via gluon splitting, as shown in figure 1 (left), has a softer $p_{\mathrm{T}}$ spectrum and a broader pseudorapidity $\eta$ distribution compared to the b jet originating from the top quark, and thus frequently escapes detection. Hence, candidate events are required to contain one muon or electron and exactly two jets, of which one is b tagged, in the final state.

In this paper, a measurement of $m_{\mathrm{t}}$ is reported based on data recorded at $\sqrt{s}=$ $13 \mathrm{TeV}$ by the CMS experiment in 2016 and corresponding to an integrated luminosity of $35.9 \mathrm{fb}^{-1}$ [21]. The study uses a sample enriched in $t$-channel single top quark events selected via a multivariate analysis (MVA) technique, and supersedes the earlier CMS measurement at $8 \mathrm{TeV}[22]$. The masses of the top quark and antiquark are also measured separately using events with positively and negatively charged leptons in the final state, respectively. The mass ratio and difference from these measurements are used to test $C P T$ invariance. Violation of $C P T$ symmetry would imply a nonlocal field theory [23], signaling physics beyond the SM. Tabulated results are provided in HEPData [24].

The paper is organized as follows. The CMS detector is briefly discussed in section 2 . Section 3 describes the simulation samples used to model signal and background events. Section 4 provides an overview of the reconstruction and identification of physics objects, as well as of the selection of candidate events. A method relying on sideband (SB) data used to estimate the QCD multijet background is described in section 5. Section 6 discusses the MVA technique designed to distinguish the $t$-channel single top quark signal from the $t \bar{t}$, EW, and QCD backgrounds. The maximum-likelihood (ML) fit used to extract the value of $m_{\mathrm{t}}$ from its reconstructed distribution is explained in section 7 . In section 8 we describe various systematic sources affecting the measurement and their individual contributions. Results and overall impact are explained in section 9, and section 10 summarizes the main findings.

\section{The CMS detector}

The central feature of the CMS apparatus is a superconducting solenoid of $6 \mathrm{~m}$ diameter, providing an axial magnetic field of $3.8 \mathrm{~T}$. Within the solenoid volume lie a silicon pixel and microstrip tracker, a lead tungstate crystal electromagnetic calorimeter (ECAL), and a brass and plastic scintillator hadron calorimeter, each composed of a barrel and two endcap sections. Forward calorimeters, based on steel absorbers with quartz fibers, extend the fiducial coverage provided by the barrel and endcap detectors up to $|\eta|=5$. Muons are measured in the range $|\eta|<2.4$, with detection planes made using three technologies: drift tubes, cathode strip chambers, and resistive plate chambers, embedded in the steel 
flux-return yoke outside the solenoid. A detailed description of the CMS detector, together with a definition of the coordinate system used and relevant kinematic variables, can be found in ref. [25].

Events of interest are selected with a two-tiered trigger system [26]. The first level [27], composed of custom hardware processors, uses information from the calorimeters and muon detectors to select events at a rate of $100 \mathrm{kHz}$. The second level, known as the high-level trigger, comprises a farm of processors running a version of the full event reconstruction software optimized for fast processing at a rate of about $1 \mathrm{kHz}$.

\section{Simulation of events}

We simulate signal and background events to NLO QCD accuracy using either the POWHEG [28-30] or MADGRAPH5_amC@NLO 2.2.2 [31] generator. The signal process [32] is simulated with POWHEG 2.0 in the four-flavor scheme (4FS), where b quarks are produced via gluon splitting as shown in figure 1 (left). This scheme is expected to yield a more accurate description of the kinematic distributions of $t$-channel events than the fiveflavor scheme (5FS) [33-35] indicated in figure 1 (right). For the normalization of signal samples, we employ the 5FS predictions calculated using HATHOR $2.1[17,18]$ at NLO in perturbative QCD. This is because with the inclusion of both gluon- and b-quark-initiated diagrams, the 5FS cross section calculation yields a more accurate value than that of $4 \mathrm{FS}$, which is based on the gluon-initiated diagram only.

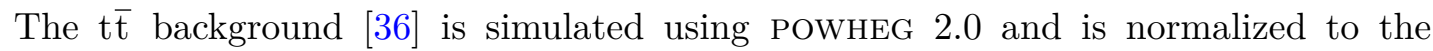
cross section calculated with TOP ++2.0 [37] at next-to-NLO (NNLO) in perturbative QCD, including soft gluon resummation at next-to-next-to-leading-log (NNLL) accuracy. We use POWHEG 1.0 to simulate the production of single top quarks in association with $\mathrm{W}$ bosons (tW) in the 5FS [38], and normalize the events to a prediction providing approximate NNLO accuracy $[39,40]$. The $s$-channel contribution is modeled using MADGRAPH5_aMC@NLO 2.2.2 in the 4FS with up to one additional parton and with the FxFx merging scheme [41] and normalized to a cross section calculated at NLO QCD accuracy in $5 \mathrm{FS}$ by HATHOR $2.1[17,18]$. While the $m_{\mathrm{t}}$ value is set to $172.5 \mathrm{GeV}$ in the nominal simulation samples of signal and top quark background events, a number of such samples generated with alternate $m_{\mathrm{t}}$ hypotheses ranging between 169.5 and $178.5 \mathrm{GeV}$ are considered for the purpose of mass calibration (section 7). In all these samples the top quark width is set to its nominal value of $1.31 \mathrm{GeV}$.

Events with $\mathrm{W}$ and $\mathrm{Z}$ bosons produced in association with jets (referred to as $\mathrm{V}+$ jets) are simulated at NLO accuracy using MADGRAPH5_aMC@NLO 2.2.2 and the FxFx merging scheme. Predictions calculated with FEWZ 3.1 [42-44] are employed for the normalization of these two processes. The $\mathrm{W}+$ jets events are simulated with zero, one, and two additional partons exclusively, in order to retain a sufficient number of events surviving the selection criteria, while the $\mathrm{Z}+$ jets events are generated with up to two additional partons inclusively. Contributions from WW, WZ, and ZZ (collectively referred to as VV) processes are simulated at NLO with MADGRAPH5_amC@NLO 2.2.2 with the FxFx merging scheme. 


\begin{tabular}{|c|c|c|c|}
\hline Process & Simulation & Cross section $(\mathrm{pb})$ & Accuracy \\
\hline Single top $t$-channel, $\mathrm{t}$ & POWHEG $2.04 \mathrm{FS}$ & 136 & NLO, estimated using HATHOR $2.1[17,18]$ in $5 \mathrm{FS}$ \\
\hline Single top $t$-channel, $\overline{\mathrm{t}}$ & POWHEG $2.04 \mathrm{FS}$ & 81 & NLO, estimated using НАТНОR $2.1[17,18]$ in $5 \mathrm{FS}$ \\
\hline Single top $s$-channel, $\mathrm{t}+\overline{\mathrm{t}}$ & MADGRAPH5_aMC@NLO 2.2.2 4FS & 10 & NLO, estimated using HATHOR $2.1[17,18]$ in $5 \mathrm{FS}$ \\
\hline $\mathrm{tW}(\overline{\mathrm{t}} \mathrm{W})$ & POWHEG 1.0 5FS & $36(36)$ & Approximate NNLO [50] \\
\hline $\mathrm{t} \overline{\mathrm{t}}$ & POWHEG 2.0 & 832 & NNLO + NNLL, estimated using TOP $++2.0[37]$ \\
\hline $\mathrm{W}(\rightarrow l \nu)+0$ jet & MADGRAPH5_aMC@NLO 2.2.2 & 50132 & NNLO, estimated using FEWZ $3.1[42-44]$ \\
\hline $\mathrm{W}(\rightarrow l \nu)+1$ jet & MADGRAPH5_aMC@NLO 2.2.2 & 8426 & NNLO, estimated using FEWZ 3.1 [42-44] \\
\hline $\mathrm{W}(\rightarrow l \nu)+2$ jets & MADGRAPH5_aMC@NLO 2.2.2 & 3173 & NNLO, estimated using FEWZ 3.1 [42-44] \\
\hline $\mathrm{Z}(\rightarrow l l)+$ jets, $\left(m_{l l}>50 \mathrm{GeV}\right)$ & MADGRAPH5_aMC@NLO 2.2.2 & 5765 & NNLO, estimated using FEWz 3.1 [42-44] \\
\hline $\mathrm{WW} \rightarrow l \nu \mathrm{qq}$ & MADGRAPH5_aMC@NLO 2.2.2 & 46 & NLO \\
\hline $\mathrm{WZ} \rightarrow l \nu \mathrm{qq}$ & MADGRAPH5_aMC@NLO 2.2.2 & 11 & NLO \\
\hline $\mathrm{ZZ} \rightarrow l l \mathrm{qq}$ & MADGRAPH5_aMC@NLO 2.2.2 & 3 & NLO \\
\hline
\end{tabular}

Table 1. Summary of signal and background simulations discussed in section 3 with their respective cross sections.

For all samples, PYTHIA 8.212 [45] is used to simulate parton showering and hadronization. We model the underlying event (UE) activities with the tune CUETP8M1 [46] for

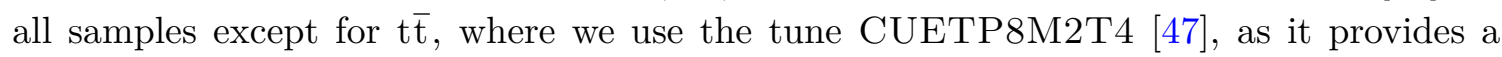
more accurate description of the kinematic distributions of the top quark pair and jets in $\mathrm{t} \overline{\mathrm{t}}$ events. The parton distribution functions (PDFs) predicted by NNPDF3.0 NLO [48] are used in all simulations. The cross sections of simulated signal and background processes are listed in table 1.

All generated events undergo a full simulation of the detector response using a model of the CMS detector implemented in GEANT4 [49]. Additional pp interactions within the same or nearby bunch crossings (pileup) included in the simulation are reweighted such that the corresponding multiplicity distribution agrees with that observed in data.

\section{Event reconstruction}

\subsection{Event selection}

Events in the muon final state are selected using a trigger that requires at least one isolated muon with $p_{\mathrm{T}}>24 \mathrm{GeV}$ and $|\eta|<2.4$. For the electron final state, the trigger requires the presence of at least one isolated electron with $p_{\mathrm{T}}>32 \mathrm{GeV}$ and $|\eta|<2.1$.

Events with at least one reconstructed pp interaction vertex are retained for further analysis. The vertex must be reconstructed from at least four tracks that have a longitudinal distance $\left|d_{z}\right|<24 \mathrm{~cm}$ and a radial distance $\left|d_{x y}\right|<2 \mathrm{~cm}$ from the nominal interaction point. If multiple vertices are found in an event, the one with the largest value of summed $p_{\mathrm{T}}^{2}$ of physics objects is taken as the primary pp interaction vertex. The objects are the jets, clustered with the tracks assigned to the vertex as inputs, and the associated missing transverse momentum $\vec{p}_{\mathrm{T}}^{\text {miss }}$, taken as the negative of the $\vec{p}_{\mathrm{T}}$ sum of those jets.

The particle-flow algorithm [51], which combines information from various subdetectors, is used to reconstruct the individual particles. Muon candidates must have at least one hit in the muon detector and a minimum of five hits in the silicon microstrip tracker. They are then reconstructed by a global fit to the combined information from the tracker and 
muon detector. Selected muons must have $p_{\mathrm{T}}>26 \mathrm{GeV}$ and $|\eta|<2.4$. Electron candidates are reconstructed [52] from good quality tracks in the tracker, matched to clusters in the ECAL. They are identified by applying dedicated selection criteria on nine variables related to tracking and shower shape. Electrons are required to pass the tight identification criteria [53] corresponding to an average efficiency of approximately $70 \%$ and have $p_{\mathrm{T}}>35 \mathrm{GeV}$ and $|\eta|<2.1$, while those falling into the gap between the ECAL barrel and endcap regions $(1.44<|\eta|<1.57)$ are rejected. The relative isolation $\left(I_{\text {rel }}\right)$ for a muon (electron) candidate is calculated by summing the transverse energy deposited by photons and charged and neutral hadrons within a cone of size $\Delta R=\sqrt{(\Delta \eta)^{2}+(\Delta \phi)^{2}}<0.4(0.3)$ around its direction, corrected for contributions from pileup [54], divided by its $p_{\mathrm{T}}$. The transverse energy is defined as $E \sin \theta$, where $E$ is the energy and $\theta$ is the polar angle of the energy deposit. The muon and electron candidates are required to pass the criterion $I_{\text {rel }}<0.06$.

Events containing additional muons (electrons) with $p_{\mathrm{T}}>10$ (15) GeV and $|\eta|<2.4$ (2.5) are rejected. In such cases, the criteria on lepton isolation are relaxed to $I_{\text {rel }}<0.2$ for muons and $I_{\text {rel }}<0.18(0.16)$ for electrons in the barrel (endcap) ECAL. We apply $p_{\mathrm{T}^{-}}$ and $\eta$-dependent scale factors in simulation for both selected and vetoed leptons to correct for observed differences in the lepton reconstruction efficiencies with data.

Jets are reconstructed using the anti- $k_{\mathrm{T}}$ clustering algorithm [55] with a distance parameter of 0.4, as implemented in the FASTJET package [56]. The effect of additional tracks and calorimetric energy deposits from pileup on the jet momentum is mitigated by discarding tracks identified to be originating from pileup vertices, as well as by applying an offset correction to account for residual neutral pileup contributions [57, 58]. Loose identification criteria [57] are applied to suppress jets arising from spurious sources, such as electronics noise in the calorimeters. Energy corrections are derived from simulation to bring the measured average response of jets to that of particle-level jets. In-situ measurements of the momentum balance in dijet, $\gamma+$ jets, $\mathrm{Z}+$ jets, and QCD multijet events are used to account for any residual differences in the jet energy scale (JES) between data and simulation [59]. In this analysis, jets are required to have $p_{\mathrm{T}}>40 \mathrm{GeV}$ and $|\eta|<4.7$.

A combined MVA tagging algorithm [5] is used to identify b jets, which are required to have $p_{\mathrm{T}}>40 \mathrm{GeV}$ and $|\eta|<2.4$. The efficiency to correctly identify b jets is about $55 \%$ at the chosen working point, while the misidentification probability is $0.1 \%$ for light-quark or gluon jets and $6 \%$ for charm jets. Simulated events are corrected using dedicated scale factors that account for the differences in the $\mathrm{b}$ tagging efficiencies and misidentification probabilities when compared to data. Candidate events must contain exactly two jets. For the signal sample selection, one of these jets is required to be b-tagged and the other must not satisfy the b tagging criterion (referred to as the "untagged" jet in the following discussion).

To suppress the background from QCD multijet processes, we require the transverse mass of the charged lepton plus neutrino system, defined as

$$
m_{\mathrm{T}}=\sqrt{\left(p_{\mathrm{T}, l}+p_{\mathrm{T}}^{\text {miss }}\right)^{2}-\left(p_{x, l}+p_{x}^{\text {miss }}\right)^{2}-\left(p_{y, l}+p_{y}^{\text {miss }}\right)^{2}},
$$

to exceed $50 \mathrm{GeV}$. Here, $p_{\mathrm{T}}^{\text {miss }}$ is the magnitude of $\vec{p}_{\mathrm{T}}^{\text {miss }}$, which is the negative of the $\vec{p}_{\mathrm{T}}$ sum of all reconstructed particle-flow objects in an event. The energy scale corrections 

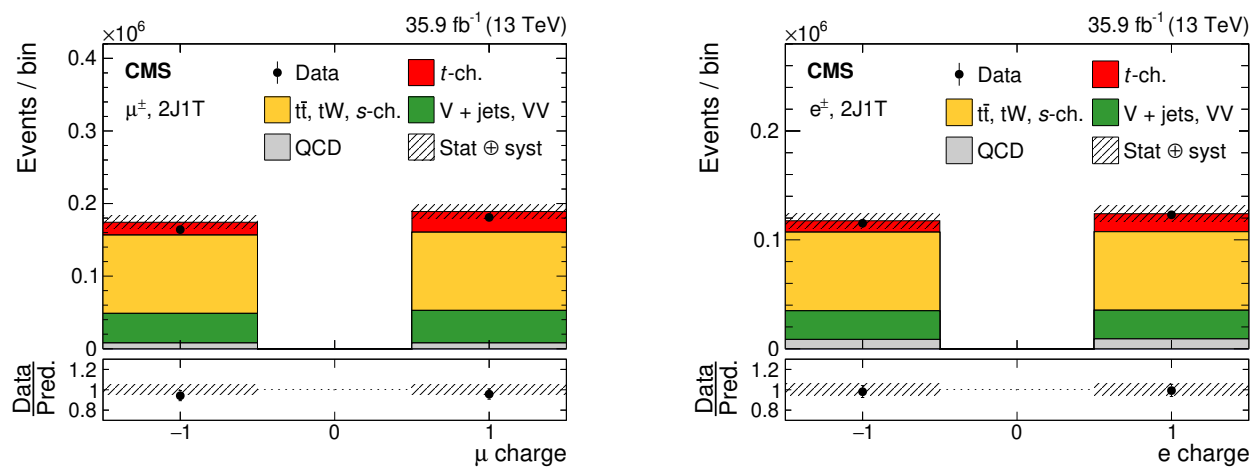

Figure 2. Event yields corresponding to positively and negatively charged muons (left) and electrons (right) in the final states obtained for the 2J1T category from data (points) and from simulated signal and background processes (colored histograms). The vertical bars on the points show the statistical uncertainty in the data, and the cross-hatched bands denote the quadrature sum of the statistical and systematic uncertainties in the predictions. The lower panels show the ratio of the data to the predictions.

applied to jets are propagated to $\vec{p}_{\mathrm{T}}^{\text {miss }}[60]$. The variables $p_{x}^{\text {miss }}$ and $p_{y}^{\text {miss }}$ denote the $x$ and $y$ components of $\vec{p}_{\mathrm{T}}^{\text {miss }}$, respectively. The symbol $p_{\mathrm{T}, l}$ represents the magnitude of $\vec{p}_{\mathrm{T}}$ of the charged lepton and $p_{x, l}\left(p_{y, l}\right)$ is its $x(y)$ component.

\subsection{Event categories}

The selected events are divided into two categories, depending on the number of jets, $n$, and number of b-tagged jets, $m$, (labeled $n \mathrm{~J} m \mathrm{~T}$ ). The $2 \mathrm{~J} 1 \mathrm{~T}$ category has the largest contribution from signal events and is referred to as the signal category. Besides this, we use the 2J0T category, where both the jets do not satisfy the $\mathrm{b}$ tagging criterion, to validate the estimation of the QCD multijet background contribution in data. A similar approach was used in earlier CMS measurements of the inclusive and differential $t$-channel single top quark cross sections $[19,35]$. The event yields in the 2J1T category after applying all selection criteria are shown in figure 2 for the muon (left) and electron (right) final states. The yields are shown separately for events with positively and negatively charged leptons. The contribution from the QCD multijet background is determined from data as described in section 5. For other processes, the event yields are obtained from simulation.

\subsection{Reconstruction of the top quark}

The four-momentum of the top quark (and hence its mass) is reconstructed from the momenta of its decay products: the charged lepton, the reconstructed neutrino, and the b-tagged jet. The momenta of the lepton and b-tagged jet are measured, while the transverse momentum of the neutrino $p_{\mathrm{T}, \nu}$ is inferred from $\vec{p}_{\mathrm{T}}^{\mathrm{miss}}$. Assuming energy-momentum conservation at the $\mathrm{W} \rightarrow l \nu$ vertex and setting the $\mathrm{W}$ boson mass $m_{\mathrm{W}}$ to $80.4 \mathrm{GeV}$ [61], the longitudinal momentum of the neutrino $p_{z, \nu}$ can be calculated from the following constraint:

$$
m_{\mathrm{W}}^{2}=\left(E_{l}+\sqrt{\left(p_{\mathrm{T}}^{\text {miss }}\right)^{2}+p_{z, \nu}^{2}}\right)^{2}-\left(\vec{p}_{\mathrm{T}, l}+\vec{p}_{\mathrm{T}}^{\text {miss }}\right)^{2}-\left(p_{z, l}+p_{z, \nu}\right)^{2},
$$


where $E_{l}$ is the lepton energy and $p_{z, l}$ is the $z$ component of its momentum. The above equation generally leads to two possible solutions for $p_{z, \nu}$, given by

$$
p_{z, \nu}=\frac{\Lambda p_{z, l}}{p_{\mathrm{T}, l}^{2}} \pm \frac{1}{p_{\mathrm{T}, l}^{2}} \sqrt{\Lambda^{2} p_{z, l}^{2}-p_{\mathrm{T}, l}^{2}\left[E_{l}^{2}\left(p_{\mathrm{T}}^{\mathrm{miss}}\right)^{2}-\Lambda^{2}\right]}
$$

where, $\Lambda=\left(m_{\mathrm{W}}^{2} / 2\right)+\vec{p}_{\mathrm{T}, l} \cdot \vec{p}_{\mathrm{T}}^{\text {miss }}$. If both solutions have real values, the one with the smaller magnitude is retained [62,63]. This choice yields a better consistency between the inferred and true values of $p_{z, \nu}$ in simulation. In the case of complex solutions, the $p_{\mathrm{T}, \nu}$ values are modified so that the radical in eq. (4.3) becomes zero, while still fulfilling eq. (4.2). Setting the radical equal to zero, we get two pairs of possible solutions for $p_{x, \nu}$ and $p_{y, \nu}$. Out of the two pairs, the one resulting in a $\vec{p}_{\mathrm{T}, \nu}$ with a lower $|\Delta \phi|$ value with respect to $\vec{p}_{\mathrm{T}}^{\mathrm{miss}}$ in the event is chosen.

The reconstructed $m_{\mathrm{t}}$ distribution after event selection for the signal and background is shown in section 6. Potential inadequacy in the determination of $p_{z, \nu}$ leads to a softer reconstructed $p_{z, \nu}$ spectrum compared to the true spectrum in simulation. This in turn leads to a mismatch of the reconstructed $m_{\mathrm{t}}$ with the true value used in simulation. A calibration is applied to the reconstructed $m_{\mathrm{t}}$ value in order to compensate for this difference, and the related uncertainty is considered as a source of systematic uncertainty, as discussed in sections 7 and 8 , respectively.

\section{Estimation of the QCD multijet background}

The QCD multijet production has a large cross section in pp collisions but a tiny acceptance in the phase space used in this analysis. Therefore, a very large sample of simulated QCD multijet events would be needed in order to retain a sufficient event yield surviving our selection criteria to ensure a reliable description of this background. In the absence of simulated event samples of the required size, an alternative approach is followed by defining an SB in data enriched in QCD multijet events. The SB is obtained by requiring the selected muon to have $0.2<I_{\text {rel }}<0.5$ and the selected electron to fail the tight identification criteria. The underlying assumption here is that the description of kinematic variables for $\mathrm{QCD}$ multijet events in the $\mathrm{SB}$ is similar to that in the signal region. We have verified this assumption using simulated samples. Shapes for QCD multijet events are derived by subtracting the total non-QCD contribution from data in this SB. As such, the SB data contain 93 (70)\% QCD multijet events for the muon (electron) final state. The QCD multijet contribution in the signal region is estimated by means of a binned ML fit to the $m_{\mathrm{T}}$ distribution with two components: QCD and non-QCD. As the QCD multijet background has a larger contribution in the 2J0T category, this category is used to validate the above method. The procedure is then applied in the signal-enriched 2J1T category where the background estimation is performed separately for positively and negatively charged leptons, as well as inclusive of the lepton charge in the final state.

Figure 3 shows the projections of the fit results onto the $m_{\mathrm{T}}$ distributions in the 2J0T (upper) and 2J1T (lower) categories for the muon (left) and electron (right) final states. To account for possible differences between QCD multijet $m_{\mathrm{T}}$ shapes obtained from the SB and 

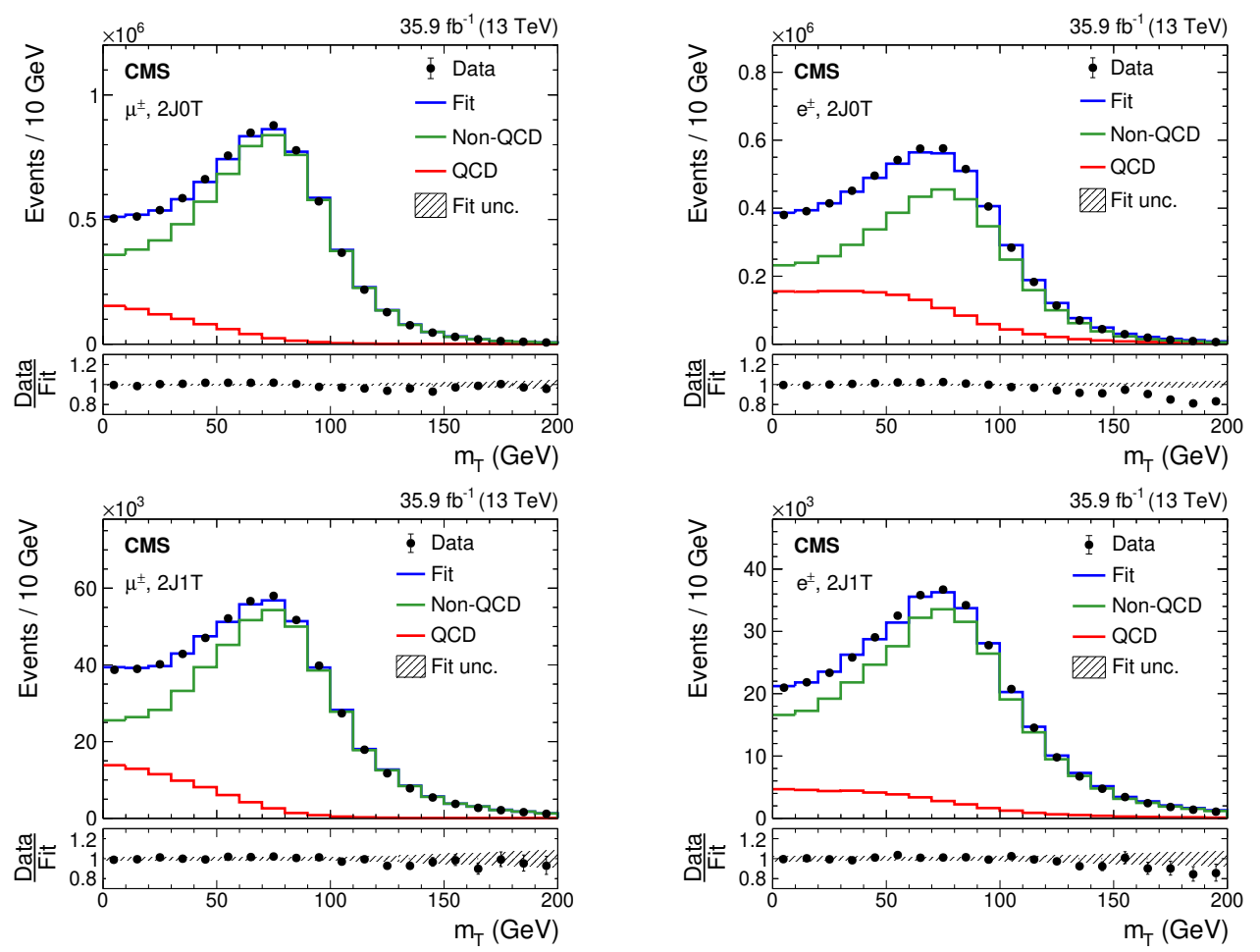

Figure 3. Projections of the fit results onto the $m_{\mathrm{T}}$ distributions for the muon (left) and electron (right) final states in the 2J0T (upper) and 2J1T (lower) categories for the data (points) and the various components of the fit (colored lines). The lower panels show the ratio of the data to the fit predictions. The bands represent the uncertainty in the $m_{\mathrm{T}}$ distribution predicted by the fit.

that in the signal region, a separate $50 \%$ systematic uncertainty is assigned to that background rate and shape (see section 8.1). This propagated uncertainty is twice the maximum difference between the data and the prediction found in the tail of the $m_{\mathrm{T}}$ distributions.

\section{Multivariate analysis}

A number of variables are combined into an MVA discriminant to separate $t$-channel single top quark events as the signal from the rest as the background. The latter is the sum total of top quark ( $\mathrm{t} \overline{\mathrm{t}}, \mathrm{tW}$, and $s$-channel), EW (V+jets and $\mathrm{VV})$, and QCD multijet events selected in the $2 \mathrm{~J} 1 \mathrm{~T}$ category. All background contributions are obtained with simulation samples except for the QCD multijet where the SB data are used, as described in section 5. We develop two boosted decision trees (BDTs) using the TMVA package [64] with the variables listed in table 2 as inputs for the muon and electron final states in the 2J1T category, respectively.

Figure 4 shows a comparison of the data and simulation for the two highest-ranked variables in table 2 . The correlations among the variables are also taken into account by using the decorrelation method available in TMVA during evaluation of the BDT response. The modeling of the other input variables in data and simulation, along with their corre- 


\begin{tabular}{|cccl}
\hline & \multicolumn{2}{c|}{ Rank } & \\
Variable & Muon Electron & Description \\
\hline$\Delta R_{\mathrm{bj}^{\prime}}$ & 1 & 1 & Angular separation in $(\eta, \phi)$ space between the b-tagged and untagged jets \\
Untagged jet $|\eta|\left(\left|\eta_{\mathrm{j}^{\prime}}\right|\right)$ & 2 & 2 & Absolute pseudorapidity of the untagged jet \\
$m_{\mathrm{bj}^{\prime}}$ & 3 & 3 & $\begin{array}{l}\text { Invariant mass of the system comprising the b-tagged and untagged jets } \\
\cos \theta^{*}\end{array}$ \\
$m_{\mathrm{T}}$ & 4 & 4 & $\begin{array}{l}\text { Cosine of the angle between the lepton and untagged jet in the rest frame } \\
\text { of the top quark }\end{array}$ \\
$\mathrm{FW} 1$ & 5 & 5 & Transverse mass as defined in eq. (4.1) \\
$\left|\Delta \eta_{l \mathrm{~b}}\right|$ & 6 & 7 & Airst-order Fox-Wolfram moment $[65,66]$ (electron final state) \\
$p_{\mathrm{T}}^{\mathrm{b}}+p_{\mathrm{T}}^{\mathrm{j}^{\prime}}$ & 7 & 8 & Scalar sum of the $p_{\mathrm{T}}$ of the b-tagged and untagged jets \\
$\left|\eta_{l}\right|$ & 8 & - & Absolute pseudorapidity of the lepton (muon final state)
\end{tabular}

Table 2. BDT input variables ranked in decreasing order of their $S P$ values for the muon and electron final states in the 2J1T category.

lations in signal and background events before and after applying the decorrelation, are presented in appendix A.

The input variables to the BDTs are chosen keeping in mind the following aspects:

- good separation power to discriminate signal from background;

- low correlation with the reconstructed $m_{\mathrm{t}}$.

The variables listed in table 2 constitute a minimal set satisfying the above conditions in the muon and electron final states, and are selected as the BDT input variables. They are ranked in decreasing order of their separation power $(S P)$ in the respective final states. The $S P$ of a given variable $x$ is defined as

$$
S P^{2}=\frac{1}{2} \int \frac{\left[\hat{X}_{S}(x)-\hat{X}_{B}(x)\right]^{2}}{\hat{X}_{S}(x)+\hat{X}_{B}(x)} \mathrm{d} x,
$$

where $\hat{X}_{S}\left(\hat{X}_{B}\right)$ denotes the probability density of $x$ in the signal (background) event category.

During training, signal and background events are weighted according to their relative contribution in the $2 \mathrm{~J} 1 \mathrm{~T}$ category. In total, 400 decision trees with a depth of three layers per tree are combined into a forest. The adaptive boosting algorithm [67] implemented in TMVA is used with the learning rate and minimum node size set to $40 \%$ and $1 \%$, respectively. The BDT setup is checked for overtraining by dividing the MC samples into two independent subsamples of equal size, one for training and the other to validate its performance. Signal and background events are picked randomly to set up the training and validation subsamples in order to avoid bias. We perform Kolmogorov-Smirnov (KS) tests to compare the BDT output distribution between the two subsamples, and obtain KS probabilities ranging between 57 and $93 \%$ for the signal and background events. These results confirm no significant overtraining. Therefore, we combine the training and validation subsamples for further studies and evaluate the BDT responses for all signal and background events according to the probabilities obtained from training in the respective final states. 

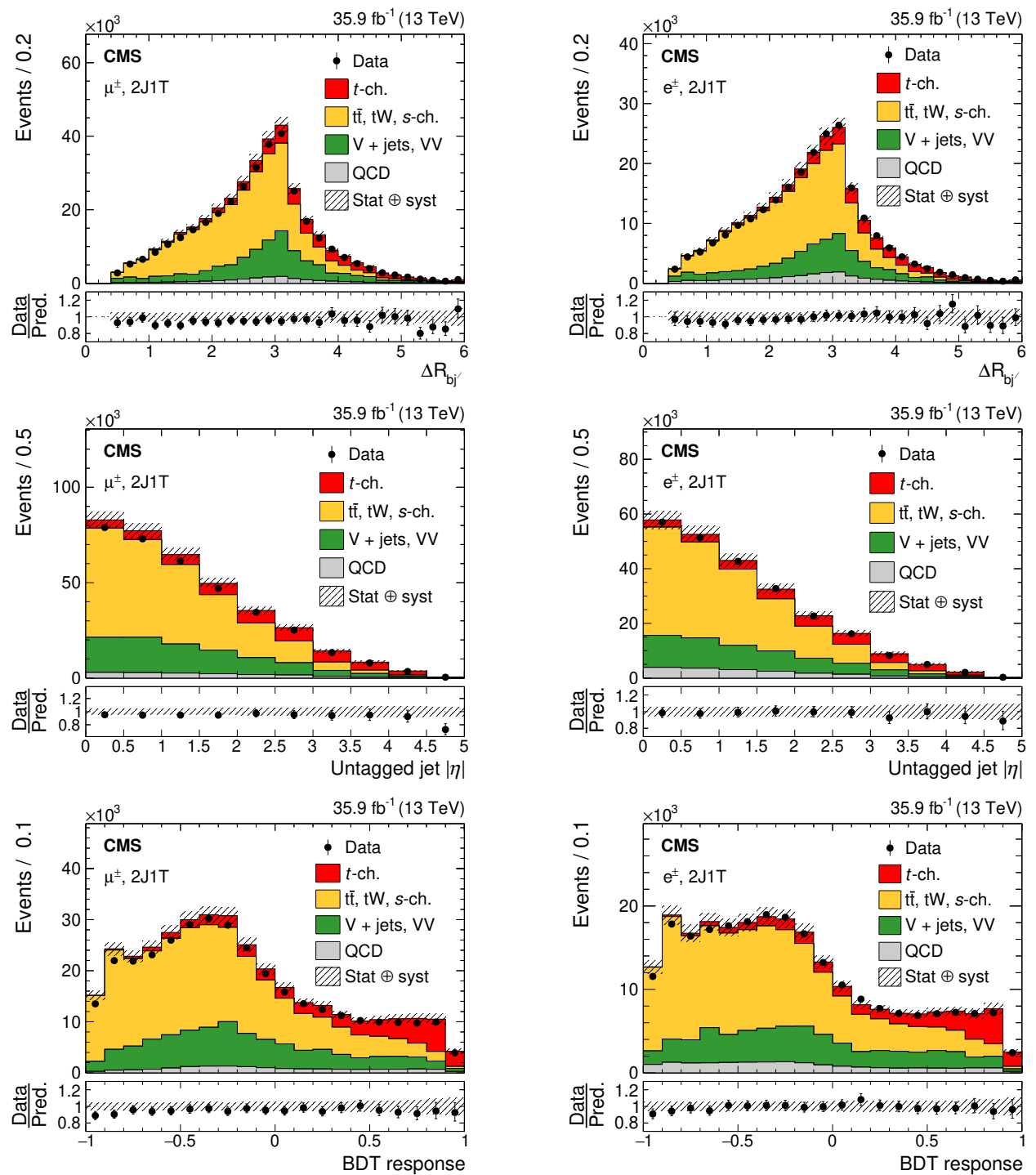

Figure 4. Data and simulation comparison for $\Delta R_{\mathrm{bj}^{\prime}}$ (upper row), untagged jet $|\eta|$ (middle row), and BDT response (lower row) in the $2 \mathrm{~J} 1 \mathrm{~T}$ category for the muon (left) and electron (right) final states. The lower panels show the ratio of the data to simulation predictions. The bands indicate the statistical and systematic uncertainties added in quadrature. The last bin in each of the upperrow plots includes the overflow.

The data-to-simulation comparisons of the BDT response distributions are shown in figure 4, separately for the muon (left) and electron (right) final states in the 2J1T category. The performance of the BDTs is quantified via a combined receiver-operator-characteristic (ROC) curve, as shown in figure 5 (upper left). The area between the ROC curve and the horizontal axis is $\approx 0.16$. A smaller area indicates a better separation between the signal and background. The correlation between the reconstructed $m_{\mathrm{t}}$ and BDT response is about $-13 \%$ in simulated signal events. 



Figure 5. Combined BDT performance in terms of the ROC curve (upper left), and the signal and background efficiencies and signal purity as functions of the BDT selection threshold (upper right) in the muon and electron final states. The uncertainty in $m_{\mathrm{t}}$ from the combined statistical and profiled systematic components (red curve), and from the mass calibration (blue curve) as a function of the BDT selection threshold (lower left). Arrows on the plots indicate the region of better separation (upper left) and optimized selection criteria (upper right and lower left). A comparison of the reconstructed $m_{\mathrm{t}}$ shapes from simulated signal events for different selection thresholds on the BDT response is shown on the lower right. The lower panel shows the ratio relative to the case where no selection (red) is applied, with the grey band denoting the normalization uncertainty around the red curve.

To obtain an event sample enriched in $t$-channel single top quark events, selection thresholds on the BDT responses are optimized by studying the signal and background efficiencies, together with the signal purity after selection, as shown in figure 5 (upper right). Ideally, high signal purity with high yield or efficiency is desirable for a precise $m_{\mathrm{t}}$ measurement in $t$-channel single top quark events. In reality, however, the signal efficiency and purity have opposite trends with increasing selection thresholds. The optimal working point is determined by studying the combined contribution of the statistical and profiled systematic uncertainties (section 8) along with that of the mass calibration, in the $m_{\mathrm{t}}$ value as a function of the BDT thresholds applied to both muon and electron final states in simulated events. These uncertainties are evaluated by means of an ML fit discussed in section 7 , based on pseudo-experiments derived from simulated events. The result of this study is presented in figure 5 (lower left). The starting point for the optimization is 
chosen to be 0.6 , where about $50 \%$ signal purity is observed for both final states. The mass calibration uncertainty reaches its minimum when the selection threshold is at 0.8 and thus offers an optimum point where the relative impact of background contamination is at its lowest. Beyond this point, both uncertainties start to increase rapidly with higher threshold values due to the depletion of the signal events. The criterion BDT response $>0.8$ is chosen, which yields about $65(60) \%$ signal purity in the muon (electron) final state.

Because of the low correlation between the BDT response and the reconstructed $m_{\mathrm{t}}$ in the signal events, a selection on the former does not alter the $m_{\mathrm{t}}$ distribution significantly, as shown in figure 5 (lower right). The differences in signal $m_{\mathrm{t}}$ shapes, obtained with various selection criteria on the BDT responses, are mostly covered by the normalization uncertainty around the peak. This is evident in the lower panel of the plot where a comparison of shape ratios obtained with different selection thresholds relative to the one without any selection on the BDT response is shown. The differences, observed in the higher tails of the $m_{\mathrm{t}}$ distributions with and without the optimized BDT selection, are covered by the signal shape variation discussed in section 8.2. The $m_{\mathrm{t}}$ distribution in data and the simulated signal and background before and after the application of the BDT selection criteria are compared in figure 6.

\section{$7 \quad$ Extraction of the top quark mass}

The high skewness of the $m_{\mathrm{t}}$ distribution, coupled with the low background rate after the BDT selection, poses a considerable challenge in obtaining appropriate analytic shapes for both the signal and background. Instead, a suitable alternative is found using the variable $\zeta=\ln \left(m_{\mathrm{t}} / 1 \mathrm{GeV}\right)$. The natural logarithm significantly reduces the skewness of the $m_{\mathrm{t}}$ distribution since more extreme values to the right of the peak are pulled in, and those to the left are stretched further away [68]. The transformed probability density functions are well-behaved since this is a monotonic one-to-one mapping.

The $\zeta$ distributions obtained from the muon and electron final states are considered in a simultaneous ML fit [69]. The fit is separately performed on events with a positively charged lepton $\left(l^{+}\right)$, negatively charged lepton $\left(l^{-}\right)$, and inclusive of the lepton charge $\left(l^{ \pm}\right)$in the final state. The inclusion of the QCD multijet background as an additional component in the fit would require a reliable parameterization, which turns out to be challenging in our case. Instead, this background contribution is subtracted from data before the fit. The QCD-subtracted binned $\zeta$ distribution is described by a analytic model $F(\zeta)$ for each final state $(l=\mu$ or e). The total likelihood is given as

$$
\mathcal{L}_{\text {tot }}=\prod_{l} \mathcal{L}_{l} \quad \text { with } \quad \mathcal{L}_{l}=\prod_{i, j} \mathcal{P}\left[N_{i, l}^{\text {obs }} \mid F_{l}\left(\zeta ; \zeta_{0}, f_{j}\right)\right] \Theta\left(f_{j}\right)
$$

where $i$ is the bin index, $\zeta_{0}$ is the peak position of the $\zeta$ distribution, $\mathcal{P}$ denotes the probability of the analytic model, $F_{l}\left(\zeta ; \zeta_{0}, f_{j}\right)$ to describe the observed $\zeta$ distribution, and $\Theta$ is the penalty term to account for the nuisance parameters $f_{j}$. These parameters are defined 

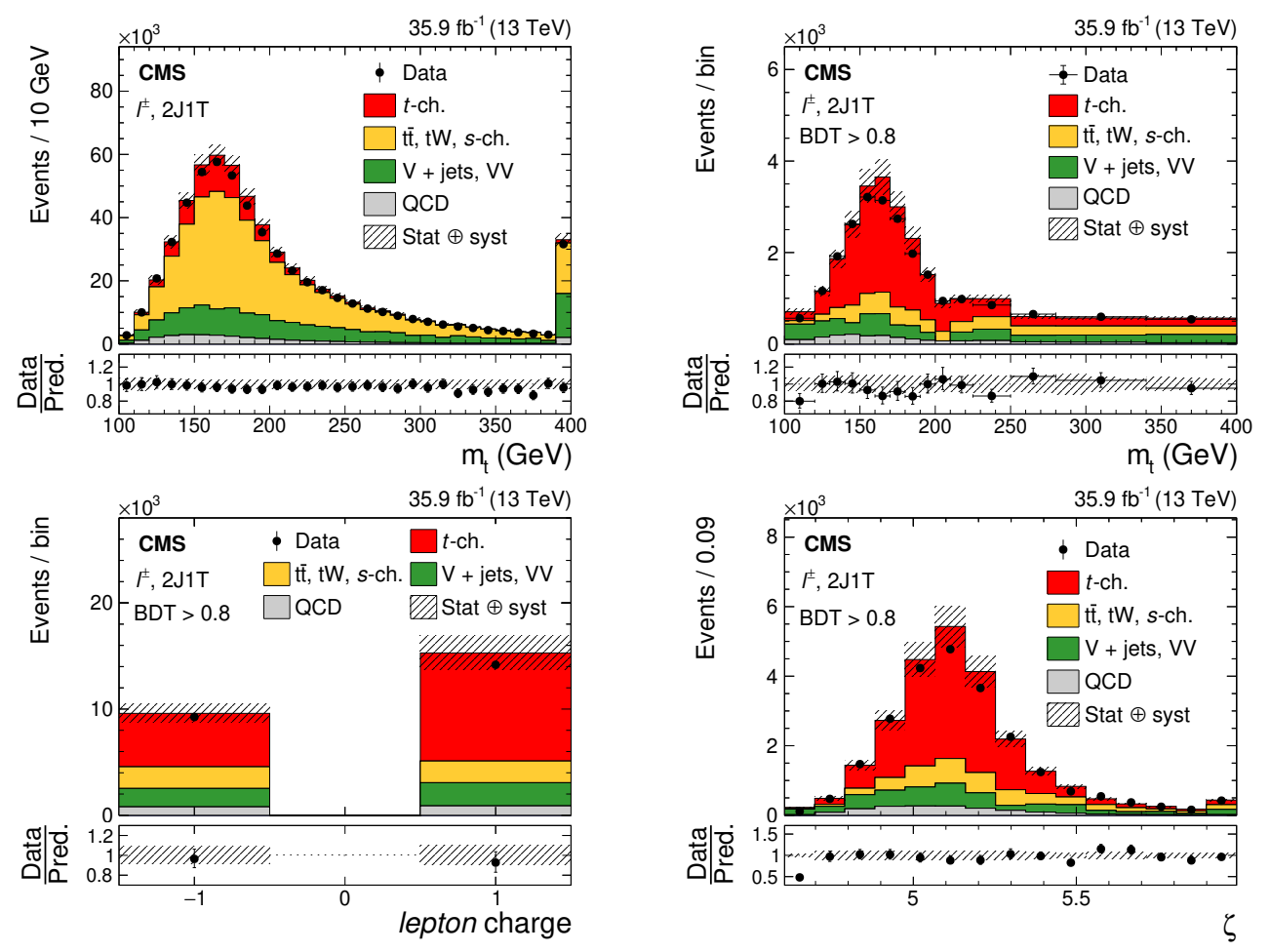

Figure 6. Reconstructed $m_{\mathrm{t}}$ distributions before (upper-left) and after (upper-right) applying the optimized BDT selection from data (points) and simulation (colored histograms) are shown in the upper row. The lower-left plot shows the event yields per lepton charge in data and simulation after optimized BDT selection. Data-to-simulation comparison of the fit variable $\zeta=\ln \left(m_{\mathrm{t}} / 1 \mathrm{GeV}\right)$ inclusive of lepton charge after optimized BDT selection is presented in the lower right plot. The horizontal bars in the upper-right plot indicate the variable bin width. The first and last bins include the underflow and overflow, respectively, for each plot. The bands denote a quadrature sum of the statistical and systematic uncertainties in the prediction. The lower panels show the ratio of the data to the prediction. The deviation from unity seen in the first bins of the upper-right and lower-right ratio plots arises because of significantly less underflow events in the data compared to the simulation.

for the rates of various event components $j$, namely signal, t $\overline{\mathrm{t}}$, and EW backgrounds, as

$$
f_{j}=\frac{N_{j}^{\mathrm{obs}}}{N_{j}^{\exp }}, \quad j \in\{\operatorname{sig}, \mathrm{t} \overline{\mathrm{t}}, \mathrm{EW}\},
$$

where $N_{j}^{\text {obs }}\left(N_{j}^{\exp }\right)$ is the observed (expected) yield for the event component $j$. We express $F_{l}\left(\zeta ; \zeta_{0}, f_{j}\right)$ as

$$
F_{l}\left(\zeta ; \zeta_{0}, f_{j}\right)=f_{\mathrm{sig}} F_{\mathrm{sig}}\left(\zeta ; \zeta_{0}\right)+f_{\mathrm{t} \overline{\mathrm{t}}} F_{\mathrm{t} \overline{\mathrm{t}}}\left(\zeta ; \zeta_{0}\right)+f_{\mathrm{EW}} F_{\mathrm{EW}}(\zeta),
$$

where $F_{\mathrm{sig}}, F_{\mathrm{t} \overline{\mathrm{t}}}$, and $F_{\mathrm{EW}}$ represent the analytic shapes for the signal, t $\overline{\mathrm{t}}$, and EW background, respectively. Small contributions $(6 \%)$ from the tW and $s$-channel single top quark processes are absorbed into the significantly larger (94\%) t $\bar{t}$ component in forming what we call the $\mathrm{t} \overline{\mathrm{t}}$ background above. 
The $F_{\text {sig }}$ shape is parameterized with a sum of an asymmetric Gaussian $\left(\zeta_{0}\right)$ function as the core and a Landau [70] function to model the higher tail. The $F_{\mathrm{t} \overline{\mathrm{t}}}$ shape is described by a Crystal Ball $\left(\zeta_{0}\right)$ function [71]. The $F_{\mathrm{EW}}$ shape describes contributions from the $\mathrm{V}+$ jets and diboson processes. It is modeled with a Novosibirsk function [72].

The parameter $\zeta_{0}$ of the combined signal and $t \overline{\mathrm{t}}$ background shapes is the parameter of interest (POI), and is allowed to float in the fit. We extract $m_{\mathrm{t}}$ from the best-fit $\zeta_{0}$ value. Parameters, except for the POI, that alter the signal and background shapes are fixed to their estimated values during the fit. These are obtained by fitting individual models to the respective distributions of simulated signal and background events in the muon and electron final states. Out of the shape parameters, the ones having large correlations with the POI are varied up to three standard deviations about their estimated values. The resulting uncertainty is considered as a separate systematic uncertainty in the measured mass. The nuisance parameters $f_{\mathrm{sig}}, f_{\mathrm{t} \overline{\mathrm{t}}}$, and $f_{\mathrm{EW}}$ are constrained in the fit using log-normal priors with 15,6 , and $10 \%$ widths, respectively. The constraint on $f_{\text {sig }}$ takes into account the uncertainty in the cross section of the inclusive $t$-channel single top quark production measured at $\sqrt{s}=13 \mathrm{TeV}$ [19]. The constraint on $f_{\mathrm{t} \overline{\mathrm{t}}}$ is driven by the uncertainty in the predicted $\mathrm{t} \overline{\mathrm{t}}$ production cross section [73]. The constraint on $f_{\mathrm{EW}}$ is relaxed to around three times the uncertainty in the measured $\mathrm{V}+$ jets cross sections [74, 75] in order to account for mismodeling of heavy-flavor jet production in simulation, as well as to cover the uncertainties due to the renormalization $\left(\mu_{\mathrm{R}}\right)$ and factorization $\left(\mu_{\mathrm{F}}\right)$ scales and PDF in the EW background. A similar approach was used in the measurement of the inclusive $t$-channel single top quark production cross section [19].

Projections of the fit results onto the $\zeta$ distributions for the $l^{+}, l^{-}$, and $l^{ \pm}$cases are shown in figure 7 . The lower panels in figure 7 show the normalized residuals or pulls, which are defined as the difference between the distribution in data and the one predicted by the fit, divided by the uncertainty $\Delta=\sqrt{\Delta_{\text {data }}^{2}-\Delta_{\text {fit }}^{2}}$. Here, $\Delta_{\text {data }}$ is the Poisson uncertainty in the data and $\Delta_{\text {fit }}$ is the uncertainty in the fit that includes both the statistical and profiled systematic components. Most of the pull values lie within \pm 2 for all three cases, with the maximum deviations occurring in the first $\zeta$ bin because of significantly less underflow events in the data than in the simulation.

We validate the fit model given in eq. (7.3) by applying it to a control sample defined by $-0.2<\mathrm{BDT}<0.8$, which is dominated by t $\overline{\mathrm{t}}$ events. The resulting best-fit values of $m_{\mathrm{t}}$ and the nuisance parameters are found to agree within the uncertainties with that obtained from the signal-enriched region (BDT $>0.8$ ). The scan of the profile likelihood ratio with the POI, together with the correlation among the fit parameters in the signal-enriched region for the $l^{ \pm}$case, are presented in appendix B. The fit consistency is checked by performing pseudo-experiments based on the $F(\zeta)$ model with profiled systematic uncertainties only. We do not observe any bias in the fit parameters, and find the corresponding pulls follow a Gaussian distribution having a mean and width consistent with 0 and 1.0, respectively, within their uncertainties.

The linearity of the best-fit mass $\left(m_{\mathrm{fit}}\right)$ is checked against different true mass $\left(m_{\text {true }}\right)$ hypotheses using dedicated simulation samples for signal and t $\bar{t}$ processes. A calibration is performed by applying a suitable offset to $m_{\text {fit }}$ to account for the differences relative 

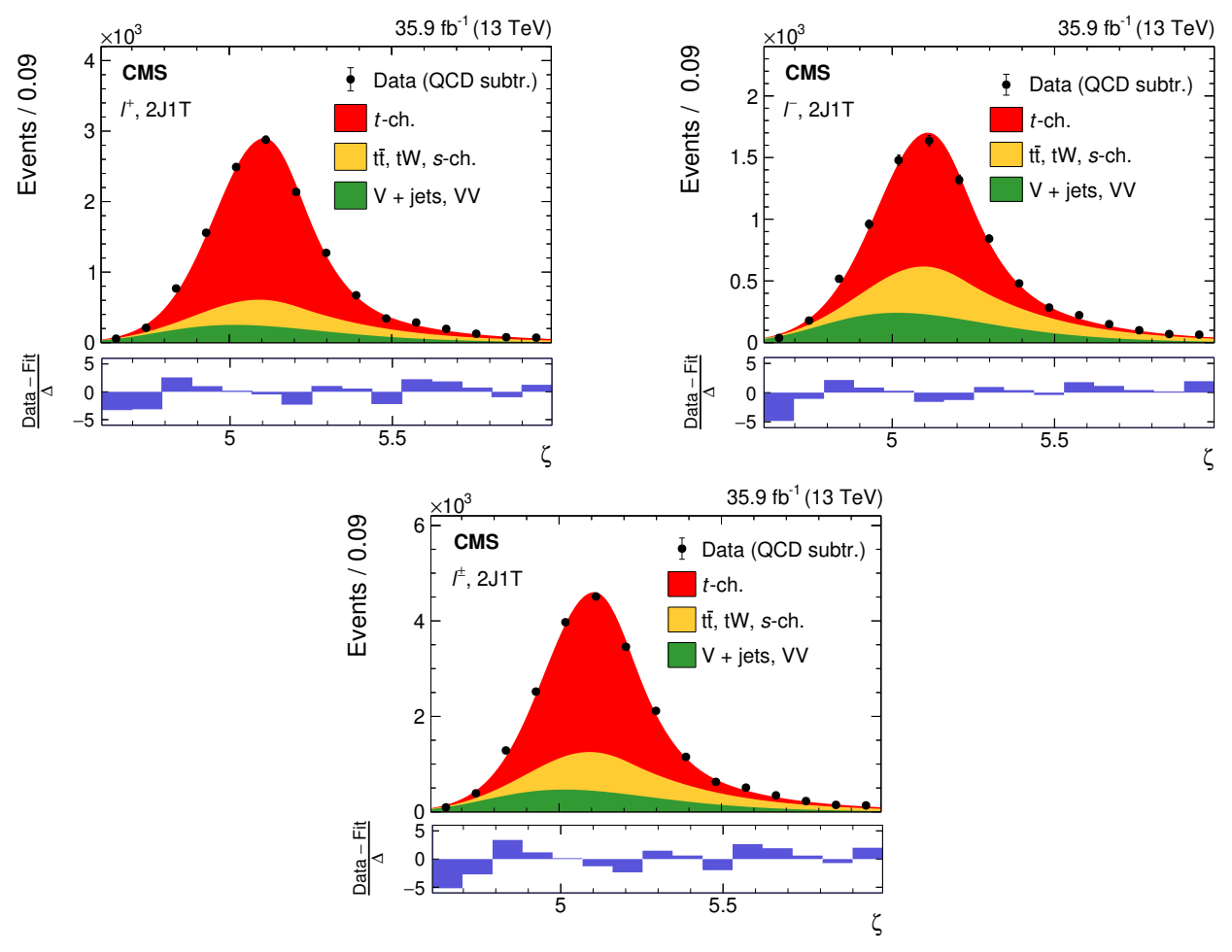

Figure 7. Projections of fit results onto the $\zeta=\ln \left(m_{\mathrm{t}} / 1 \mathrm{GeV}\right)$ distributions for the $l^{+}$(upper left), $l^{-}$(upper right), and $l^{ \pm}$(lower row) final states for signal and background processes compared to data in the 2J1T category with the optimized BDT selection applied. The lower panels show the pulls that are determined using the bin contents of the data distributions (after QCD subtraction) and the $F(\zeta)$ values evaluated at the center of the bins. The fitted $\zeta$ distributions for each final state are combined in these plots for a comparison with data for the three different cases based on the lepton charge.

to $m_{\text {true }}$. The difference between $m_{\text {fit }}$ and $m_{\text {true }}$ can be attributed to the inadequacy in the determination of $p_{z, \nu}$ in the signal process discussed in section 4.3. Details about the associated systematic uncertainty due to the mass calibration are discussed in section 8.1.

\section{Systematic uncertainties}

We consider several sources of systematic uncertainties. They can be broadly classified into two categories as follows.

1. Profiled (prof): the signal and background normalizations are profiled by including them as nuisance parameters in the ML fit. The impacts of the profiled systematic sources are obtained directly from the fit via correlations between the POI and the nuisance parameters.

2. Externalized (ext): all other uncertainty sources are externalized, i.e., the ML fit is repeated with varied $\zeta$ shapes. Thus, full variations of the shapes are considered due to these sources. The impacts of these sources are calculated by taking the 
difference between the offset corrected best-fit values of $m_{\mathrm{t}}$ corresponding to the nominal and varied shapes. The largest shift relative to the nominal result is quoted as the uncertainty for a particular systematic source in a conservative approach, unless otherwise specified. The total uncertainty due to the externalized systematic sources in the central value is obtained by separately combining the positive and negative shifts.

The externalized systematic sources can be further divided into two subcategories, namely experimental and modeling.

\subsection{Experimental uncertainties}

- JES: the energies of all reconstructed jets in simulated events are simultaneously scaled up and down according to their $p_{\mathrm{T}^{-}}$and $\eta$-dependent uncertainties [76], split into correlation groups, namely intercalibration, MPFInSitu, and uncorrelated according to the procedure described in ref. [77]. A similar approach to subcategorize the JES uncertainties was used in ref. [9] for the $m_{\mathrm{t}}$ measurement with t $\overline{\mathrm{t}}$ events in the $l+$ jets final state.

- Jet energy resolution (JER): to account for the difference in JER between data and simulation, a dedicated smearing is applied [76] in simulation that improves or worsens the resolutions within their uncertainties.

- Unclustered energy: the contributions of unclustered particles to $p_{\mathrm{T}}^{\mathrm{miss}}$ are varied within their respective energy resolutions [78].

- Muon and electron efficiencies: the lepton identification, isolation, and trigger efficiencies are determined with a "tag-and-probe" method [79] from Drell-Yan events falling in the $\mathrm{Z}$ boson mass window. The efficiency correction factors are applied to simulated events in order to match with data. The uncertainties in these correction factors are varied in bins of $p_{\mathrm{T}}$ and $|\eta|$.

- Pileup: the uncertainty in the expected distribution of pileup is propagated as a systematic uncertainty by varying the total inelastic cross section by $\pm 4.6 \%$ [80] about its central value of $69.2 \mathrm{mb}$ by reweighting the simulated events.

- $b$ tagging: the scale factors used to calculate the efficiency corrections for the $b$ tagging algorithm are varied up and down within their uncertainties. The efficiency corrections found from these variations are then applied to the simulation to estimate the corresponding systematic uncertainty.

- QCD multijet background: the contribution of the QCD multijet background is estimated based on data as discussed in section 5. Its contribution is first subtracted from data before the final fit using the parametric model given in eq. (7.3). Each bin of the QCD multijet shape derived from SB data is varied independently by an uncertainty of $50 \%$ and a new set of shapes is obtained. The resulting new shapes are subtracted from data in the signal region one at a time and the fit is repeated. In this 
method, a maximal variation in the rate and shape of the QCD multijet background is considered. The resulting uncertainty is obtained from the difference in the mean value of the offset-corrected fit results relative to the nominal case.

- Mass calibration: the mass calibration $\left(\Delta m_{\text {cal }}\right)$, i.e., the difference between $m_{\text {fit }}$ and $m_{\text {true }}$, is obtained as a function of $m_{\text {fit }}$, using dedicated MC samples with alternate $m_{\text {true }}$ hypotheses (figure 8 ). The linear behavior between $m_{\text {fit }}$ and $m_{\text {true }}$ dictates that $\Delta m_{\text {cal }}$ also has a linear dependence on $m_{\text {fit }}$. The band about the central line represents the \pm 1 standard deviation owing to statistical fluctuations of the signal and $\mathrm{t} \overline{\mathrm{t}}$ samples with different $m_{\text {true }}$ hypotheses. The mass calibration is obtained from the central value, while its uncertainty is determined from the band and considered as an independent source of uncertainty. This procedure has been implemented separately for the $l^{+}, l^{-}$, and $l^{ \pm}$cases.

- Luminosity: the relative uncertainty in the integrated luminosity is $\pm 2.5 \%$ [81]. This is propagated to the uncertainties in the expected rates of the signal and background processes except for the QCD multijet, which is determined from data.

\subsection{Modeling uncertainties}

- $C R$ and early resonance decay (ERD): the uncertainties due to ambiguities in modeling CR effects are estimated by comparing the default model in PYTHIA 8 with two alternative $\mathrm{CR}$ models, one with string formation beyond leading color ("QCD inspired") [15], and the other in which the gluons can be moved to another string ("gluon move") [16]. In addition, CR effects due to the top quark decay products are assessed by switching off (default) and on ERD [16] in PYTHIA 8. In the first case, the lifetime of the top quark is assumed to be long enough to shield its decay products from color reconnecting with the rest of the event, whereas this restriction is lifted when we enable the ERD option. All models are tuned to the UE measurements in ref. [82], and simultaneous variations of different CR models in signal and $\mathrm{t} \overline{\mathrm{t}}$ simulations are considered. The largest observed shift is quoted as the systematic uncertainty.

- Flavor-dependent JES: the Lund string fragmentation model of PYTHIA 8 [45] is compared with the cluster fragmentation of HERWIG ++ [83]. Each model relies on a large set of tuning parameters that allows one to modify the individual fragmentation of jets initiated from gluons and light, charm, and bottom quarks. Therefore, the difference in JES between PYTHIA and HERWIG is determined for each jet flavor [76]. The flavor uncertainties for jets from gluons and light, charm, and bottom quarks are evaluated separately and added linearly [9] since these individual contributions are treated to be fully correlated. This method ensures that the relevant systematic sources are varied simultaneously for all jet flavors.

- $b$ quark hadronization: this term accounts for flavor-dependent uncertainties arising from the simulation of parton fragmentation. The fragmentation of $b$ quarks into $b$ 

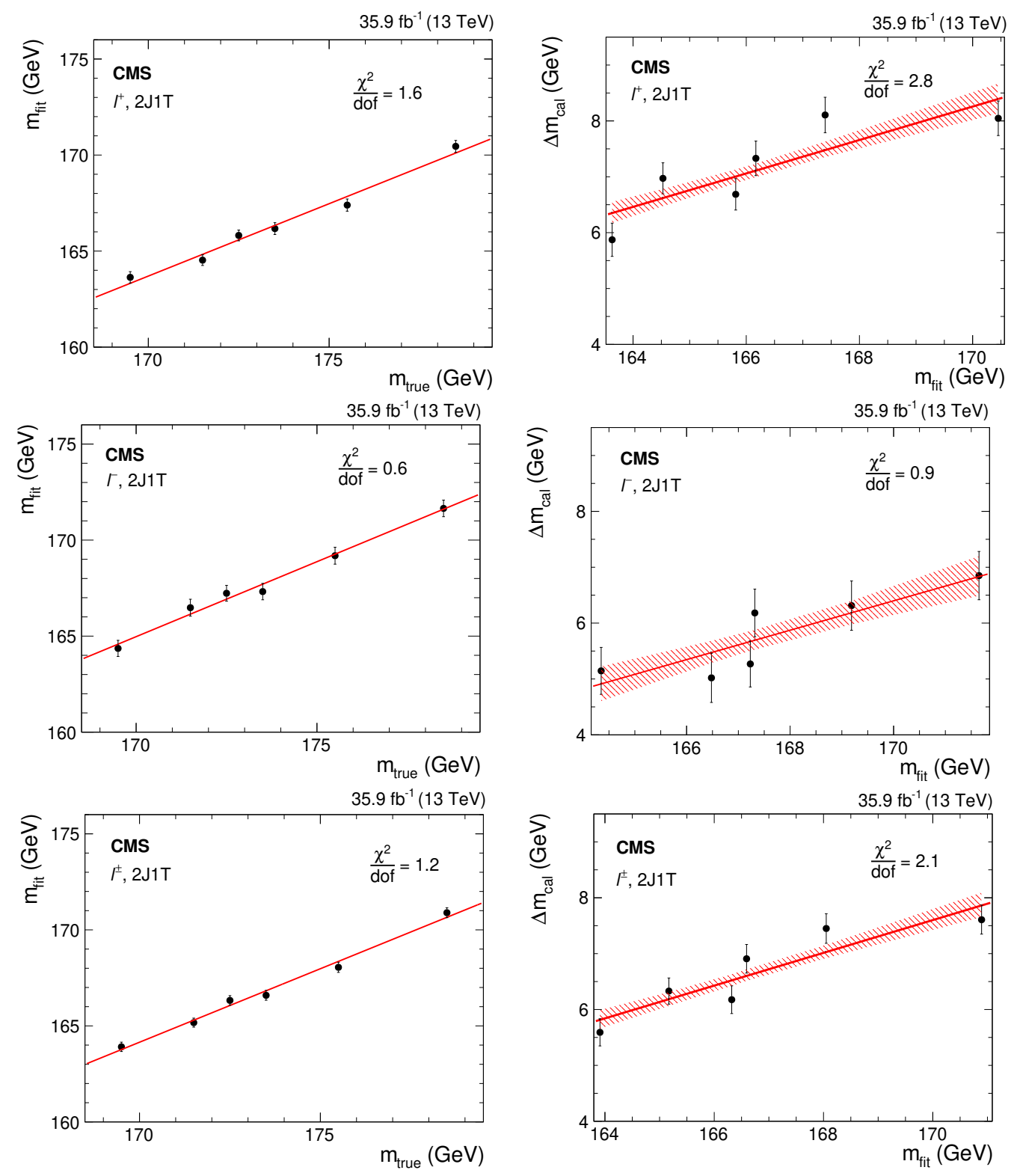

Figure 8. Test of the linearity of the $m_{\text {fit }}$ for different values of true mass $m_{\text {true }}$ (left), and the resulting mass calibration $\Delta m_{\text {cal }}$ as a function of $m_{\text {fit }}$ (right) for events in the 2J1T category for the $l^{+}$(upper), $l^{-}$(middle), and $l^{ \pm}$(lower) cases. The shaded regions indicate \pm 1 standard deviations about the central values defined by the red line. The value of the $\chi^{2}$ per degrees of freedom (dof) from the linear fit is shown in each plot. 
hadrons is varied in simulation within uncertainties in the Bowler-Lund fragmentation function tuned to the ALEPH [84], DELPHI [85], and SLD [86] data. In addition, the difference between the Bowler-Lund [87] and Peterson [88] fragmentation functions is included in the uncertainty. Lastly, the uncertainty due to the semileptonic b hadron branching fraction is obtained by varying it by $-0.45 \%$ and $+0.77 \%$, which is the range of measurements from $\mathrm{B}^{0} / \mathrm{B}^{ \pm}$decays and their uncertainties [61].

- Signal modeling: to determine the influence of possible mismodeling of the $t$-channel single top quark process, several sources are considered as listed below.

1. Parton shower $(P S)$ scale: we compare the nominal signal shape with reweighted shapes obtained by using per-event weights corresponding to independent variations of initial- and final-state radiation (ISR and FSR) scales by a factor of 2 and $1 / 2$, respectively. During estimation of the related uncertainties, the ISR scale is kept fixed at the nominal value while the FSR scale is varied and viceversa. The uncertainty is estimated from the difference in the fit results using reweighted shapes relative to the nominal one.

2. $\mu_{\mathrm{R}}$ and $\mu_{\mathrm{F}}$ scales: the impacts of varying the $\mu_{\mathrm{R}}$ and $\mu_{\mathrm{F}}$ scales up and down by a factor of 2 relative to their respective nominal values (both set to $172.5 \mathrm{GeV}$ ) are considered by applying per-event weights [89] on the $\zeta$ distributions. Two cases are considered for the evaluation of related uncertainties. In the first case, one scale is varied while the other is kept fixed to its nominal value; in the other case, both scales are varied together in the same direction with respect to their nominal values. The resulting uncertainties from each case are added in quadrature and quoted as the uncertainty due to the $\mu_{\mathrm{R}}$ and $\mu_{\mathrm{F}}$ scales.

3. $P D F+\alpha_{\mathrm{S}}$ : the impact due to the choice of PDFs is studied using reweighted shapes that are derived from replicas of the NNPDF $3.0 \mathrm{NLO}\left(\alpha_{\mathrm{S}}=0.118\right)$ PDF set [48]. In addition, NNPDF3.0 sets with $\alpha_{\mathrm{S}}=0.117$ and 0.119 are evaluated and the observed difference is added in quadrature.

- $t \bar{t}$ modeling: the impacts due to variation of the ISR and FSR scales, the $h_{\text {damp }}$ parameter responsible for ME-PS matching [47] (where ME is the acronym for matrix element), the $\mu_{\mathrm{R}}$ and $\mu_{\mathrm{F}}$ scales, and $\mathrm{PDF}+\alpha_{\mathrm{S}}$ in the t$\overline{\mathrm{t}}$ process are considered. The uncertainties due to the ISR and FSR scales, $\mu_{\mathrm{R}}$ and $\mu_{\mathrm{F}}$ scales, and PDF $+\alpha_{\mathrm{S}}$ variations in $t \bar{t}$ events are estimated by following exactly the same method as for

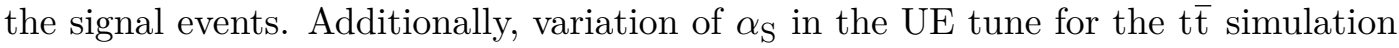
sample is considered in order to cover the difference between the UE models used for simulated $t \bar{t}$ and all other processes, as discussed in section 3. We also take into account the mismodeling of the top quark $p_{\mathrm{T}}$ spectrum, which is harder in simulated $\mathrm{t} \overline{\mathrm{t}}$ events than in the data [90]. The uncertainties due to the aforementioned sources are determined from the difference in fit results obtained from the varied or reweighted $t \bar{t}$ shapes corresponding to each source relative to the nominal one. The contributions from individual sources are then added in quadrature to obtain the total uncertainty

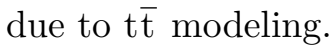


- Parametric shapes: the impact from varying the shape parameters of the signal and background models is considered as a separate systematic uncertainty. The shape parameters are varied by three standard deviations about their estimated values, derived using simulation. The impacts due to individual sources are summed in quadrature to obtain the total uncertainty due to the parametric modeling of the signal and backgrounds.

Table 3 summarizes the aforementioned sources of systematic uncertainty and their contributions. The impacts due to alternative ME (MADGRAPH5_aMC@NLO), FS (5FS), PS (HERWIG++), and UE (CUETP8M2T4) modeling of the signal process are also evaluated for the $l^{ \pm}$final state using dedicated simulated event samples. Their individual contributions range between -0.36 and $+0.16 \mathrm{GeV}$. As these values are covered by the total systematic uncertainty listed in table 3 , no additional uncertainty is assigned to the measured $m_{\mathrm{t}}$ value due to these sources.

In the case of the signal process, top quarks are produced more abundantly relative to their antiquark partners due to the charge asymmetry of the $\mathrm{W}$ boson radiated from the initial-state quark in pp collisions at the LHC. This leads to a higher relative background contamination in the $l^{-}$final state arising from top antiquark decay compared to the $l^{+}$ final state from top quark decay, as shown in figure 7. As a result, the measurement in the $l^{-}$final state is more sensitive to the sources that significantly alter the background contributions along with the signal, compared to the ones that impact the signal contribution only. This is reflected in table 3 where the uncertainties from the signal modeling are lower for the $l^{-}$case; whereas other sources, except for the ones listed under flavor-dependent JES, that alter the background contributions along with the signal have a larger impact on the total uncertainty. In the case of the flavor-dependent JES uncertainty sources, the uncertainty is primarily dictated by the untagged jet. The presence of a light quark in the final state is a salient feature of the signal process. This light quark, or the FSR gluon radiated from it, is often detected as the untagged jet in the endcap region. The untagged-jet kinematic properties are heavily exploited by the BDTs in order to achieve a better separation between the signal and backgrounds. Hence, it has a large impact on the final acceptance, as well as on the $\zeta$ shapes obtained after the BDT selection. The energy calibration of the endcap detector is known to have larger uncertainties compared to the barrel and hence it has a larger impact on the untagged jet found in the endcap region. In the $l^{-}$final state, the relative contribution of the flavor-dependent JES uncertainty is smaller owing to a lower signal-to-background ratio.

\section{Results}

The $m_{\mathrm{t}}$ value is measured with events dominated by $t$-channel single top quark process, inclusive of the lepton charge in the final state, as

$$
m_{\mathrm{t}}=172.13 \pm 0.32(\text { stat }+ \text { prof })_{-0.70}^{+0.69}(\text { ext }) \mathrm{GeV}=172.13_{-0.77}^{+0.76} \mathrm{GeV}
$$




\begin{tabular}{|c|c|c|c|c|}
\hline Source & & $\delta m_{l^{ \pm}}$ & $\delta m_{l}+$ & $\delta m_{l^{-}}$ \\
\hline Statistical & & \pm 0.19 & \pm 0.23 & \pm 0.33 \\
\hline Statistical + profiled systematic & & \pm 0.32 & \pm 0.37 & \pm 0.58 \\
\hline \multirow[t]{2}{*}{ JES } & $\begin{array}{l}\text { Correlation group intercalibration } \\
\text { Correlation group MPFInSitu } \\
\text { Correlation group uncorrelated }\end{array}$ & $\begin{array}{l} \pm 0.09 \\
\pm 0.02 \\
\pm 0.39\end{array}$ & $\begin{array}{l} \pm 0.07 \\
\pm 0.02 \\
\pm 0.17\end{array}$ & $\begin{array}{l} \pm 0.12 \\
\pm 0.01 \\
\pm 0.83\end{array}$ \\
\hline & Total (quadrature sum) & \pm 0.40 & \pm 0.18 & \pm 0.84 \\
\hline $\begin{array}{c}\text { JER } \\
\text { Unclustered energy } \\
\text { Muon efficiencies } \\
\text { Electron efficiencies } \\
\text { Pileup } \\
\text { b tagging } \\
\text { QCD multijet background } \\
\text { Mass calibration } \\
\text { Int. luminosity }\end{array}$ & & $\begin{array}{l}<0.01 \\
<0.01 \\
<0.01 \\
\pm 0.01 \\
\pm 0.14 \\
\pm 0.20 \\
\pm 0.02 \\
\pm 0.11 \\
<0.01\end{array}$ & $\begin{array}{l}<0.01 \\
<0.01 \\
<0.01 \\
\pm 0.01 \\
\pm 0.04 \\
\pm 0.18 \\
\pm 0.01 \\
\pm 0.13 \\
<0.01\end{array}$ & $\begin{array}{l}<0.01 \\
<0.01 \\
<0.01 \\
\pm 0.01 \\
\pm 0.34 \\
\pm 0.22 \\
\pm 0.02 \\
\pm 0.20 \\
\pm 0.01\end{array}$ \\
\hline CR model and ERD & & $\pm 0.24(0.017)$ & $\pm 0.39(0.027)$ & $\pm 0.68(0.048)$ \\
\hline \multirow[t]{2}{*}{ Flavor-dependent JES } & $\begin{array}{c}\text { Gluon } \\
\text { Light quark (uds) } \\
\text { Charm } \\
\text { Bottom }\end{array}$ & $\begin{array}{l}+0.52 \\
-0.18 \\
+0.01 \\
-0.48\end{array}$ & $\begin{array}{l}+0.75 \\
+0.18 \\
+0.08 \\
-0.29\end{array}$ & $\begin{array}{l}-0.03 \\
-0.23 \\
+0.11 \\
-0.31\end{array}$ \\
\hline & Total (linear sum) & -0.13 & +0.72 & -0.46 \\
\hline \multirow[t]{2}{*}{ b quark hadronization model } & $\begin{array}{l}\text { b frag. Bowler-Lund } \\
\text { b frag. Peterson } \\
\text { Semileptonic b hadron decays }\end{array}$ & $\begin{array}{l} \pm 0.03 \\
+0.14 \\
\pm 0.18\end{array}$ & $\begin{array}{l} \pm 0.06 \\
+0.11 \\
\pm 0.17\end{array}$ & $\begin{array}{l} \pm 0.08 \\
+0.19 \\
\pm 0.19\end{array}$ \\
\hline & Total (quadrature sum) & $+0.23-0.18$ & $+0.21-0.18$ & $+0.28-0.21$ \\
\hline \multirow[t]{2}{*}{ Signal modeling } & $\begin{array}{c}\text { ISR } \\
\text { FSR } \\
\mu_{\mathrm{R}} \text { and } \mu_{\mathrm{F}} \text { scales } \\
\mathrm{PDF}+\alpha_{\mathrm{S}}\end{array}$ & $\begin{array}{l} \pm 0.01 \\
\pm 0.28 \\
\pm 0.09 \\
\pm 0.06\end{array}$ & $\begin{array}{l} \pm 0.01 \\
\pm 0.31 \\
\pm 0.13 \\
\pm 0.06\end{array}$ & $\begin{array}{l}<0.01 \\
\pm 0.20 \\
\pm 0.03 \\
\pm 0.07\end{array}$ \\
\hline & Total (quadrature sum) & \pm 0.30 & \pm 0.34 & \pm 0.21 \\
\hline \multirow[t]{2}{*}{$\mathrm{t} \overline{\mathrm{t}}$ modeling } & $\begin{array}{c}\text { ISR } \\
\text { FSR } \\
\text { ME-PS matching scale } \\
\mu_{\mathrm{R}} \text { and } \mu_{\mathrm{F}} \text { scales } \\
\text { PDF }+\alpha_{\mathrm{S}} \\
\text { Top quark } p_{\mathrm{T}} \text { reweighting } \\
\mathrm{UE}\end{array}$ & $\begin{array}{l} \pm 0.11(0.008) \\
\pm 0.10(0.007) \\
\pm 0.10(0.007) \\
\quad \pm 0.03 \\
\quad<0.01 \\
\quad-0.04 \\
\pm 0.07(0.005)\end{array}$ & $\begin{array}{l} \pm 0.02(0.001) \\
\pm 0.14(0.010) \\
\pm 0.10(0.006) \\
\quad \pm 0.03 \\
<0.01 \\
\quad-0.08 \\
\pm 0.04(0.003)\end{array}$ & $\begin{array}{l} \pm 0.22(0.016) \\
\pm 0.40(0.028) \\
\pm 0.10(0.008) \\
\quad \pm 0.01 \\
\quad<0.01 \\
\quad-0.04 \\
\pm 0.17(0.012)\end{array}$ \\
\hline & Total (quadrature sum) & \pm 0.20 & $+0.18-0.20$ & \pm 0.50 \\
\hline \multirow[t]{2}{*}{ Parametric shapes } & $\begin{array}{l}\text { Signal shape } \\
\text { tE bkg. shape } \\
\text { EW bkg. shape }\end{array}$ & $\begin{array}{l} \pm 0.05 \\
\pm 0.07 \\
\pm 0.03\end{array}$ & $\begin{array}{l} \pm 0.03 \\
\pm 0.04 \\
\pm 0.01\end{array}$ & $\begin{array}{l} \pm 0.04 \\
\pm 0.05 \\
\pm 0.02\end{array}$ \\
\hline & Total (quadrature sum) & \pm 0.09 & \pm 0.05 & \pm 0.07 \\
\hline Total externalized systematic & & $+0.69-0.71$ & $+0.97-0.65$ & $+1.32-1.39$ \\
\hline Grand total & & $+0.76-0.77$ & $+1.04-0.75$ & $+1.44-1.51$ \\
\hline
\end{tabular}

Table 3. Summary of the $m_{\mathrm{t}}$ uncertainties in $\mathrm{GeV}$ for each final-state lepton charge configuration. The statistical uncertainties are obtained by performing the fits again in each case while fixing the nuisance parameters to their estimated values from data. With the exception of the flavordependent JES sources, the total systematic uncertainty is obtained from the quadrature sum of the individual systematic sources. The amount of statistical fluctuations in the systematic shifts are quoted within parentheses whenever alternative simulated samples with systematic variations have been used. These are determined from 1000 pseudo-experiments in each case. Entries with $<0.01$ denote that the magnitude of the systematic bias is less than 0.01. 
The masses of the top quark and antiquark are determined separately by requiring positively and negatively charged leptons in the final state, respectively. We find

$$
\begin{aligned}
& m_{\mathrm{t}}=172.62 \pm 0.37(\text { stat }+ \text { prof })_{-0.65}^{+0.97}(\text { ext }) \mathrm{GeV}=172.62_{-0.75}^{+1.04} \mathrm{GeV} \\
& m_{\overline{\mathrm{t}}}=171.79 \pm 0.58(\text { stat }+ \text { prof })_{-1.39}^{+1.32}(\text { ext }) \mathrm{GeV}=171.79_{-1.51}^{+1.44} \mathrm{GeV}
\end{aligned}
$$

The first uncertainty is the combination of the statistical and profiled systematic uncertainties, whereas the second denotes the uncertainty due to the externalized systematic sources. The total uncertainty is obtained by adding the two values in quadrature. The measured masses of the top quark and antiquark are consistent with each other, as well as with the $l^{ \pm}$result, within uncertainties. The measured value corresponding to the charge-inclusive final states agrees with previous measurements by the ATLAS [7] and CMS [8-10, 14, 22] Collaborations at different center-of-mass energies using various final states, within the uncertainties. Comparisons of the previous measurements and the result from this analysis are shown in figure 9 .

The mass ratio of the top antiquark to quark is determined to be

$$
R_{m_{\mathrm{t}}}=\frac{m_{\overline{\mathrm{t}}}}{m_{\mathrm{t}}}=0.9952 \pm 0.0040(\text { stat }+ \text { prof })_{-0.0096}^{+0.0068}(\text { ext })=0.9952_{-0.0104}^{+0.0079},
$$

and the mass difference between the top quark and antiquark is

$$
\Delta m_{\mathrm{t}}=m_{\mathrm{t}}-m_{\overline{\mathrm{t}}}=0.83 \pm 0.69(\text { stat }+ \text { prof })_{-1.16}^{+1.65}(\text { ext }) \mathrm{GeV}=0.83_{-1.35}^{+1.79} \mathrm{GeV}
$$

The uncertainties in the mass ratio and difference are obtained by combining the uncertainties from the individual measurements, as indicated in eqs. (9.2) and (9.3). Here, it is assumed that the combined statistical and profiled systematic uncertainties in the top quark and antiquark masses are uncorrelated, while the externalized systematic uncertainties are fully correlated source by source. The estimated values of $\mathrm{R}_{m_{\mathrm{t}}}$ and $\Delta m_{\mathrm{t}}$ are consistent with unity and zero, respectively, within uncertainties, showing no evidence for violation of $C P T$ invariance. Figure 10 compares $\Delta m_{\mathrm{t}}$ from this analysis with the previous measurements by the ATLAS [91] and CMS [92] Collaborations in t $\overline{\mathrm{t}}$ events at $\sqrt{s}=7$ and $8 \mathrm{TeV}$, respectively. All results agree with each other and are consistent with zero, within the uncertainties.

The precision of the $m_{\mathrm{t}}$ measurement presented here shows about a $30 \%$ improvement over the previous CMS result [22] from single top quark events. The inclusion of the electron final state improves the overall signal yield, thus reducing the statistical component of the total uncertainty. The MVA discriminant and the nuisance parameters in the ML fit constrain the background contamination to a level where the impact of dominant systematic uncertainty sources including JES can be brought under control. These improved strategies are responsible for reducing the overall uncertainty in the measured mass. The statistical uncertainty plays a minor role in the achieved precision, which is limited by the systematic uncertainties due to JES, CR, and FSR modeling in the signal process. A deeper understanding of these effects would be needed to further improve the precision. 


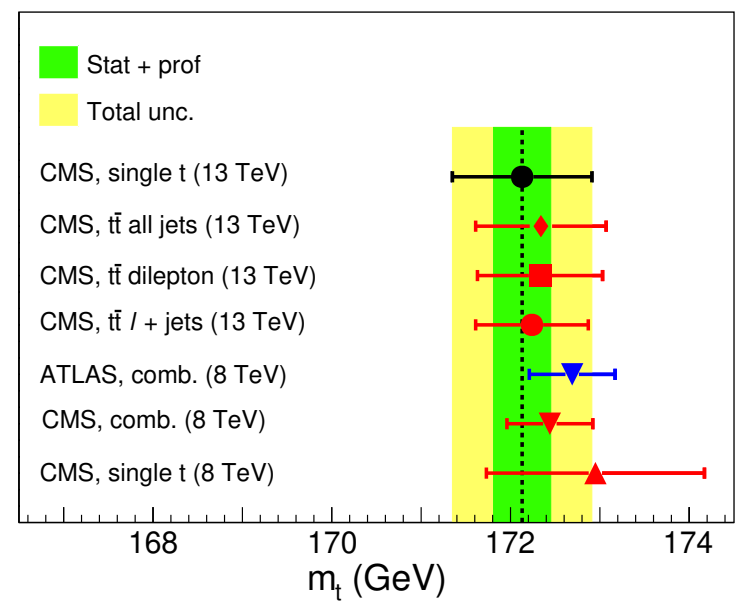

Figure 9. A comparison of measured $m_{\mathrm{t}}$ values from this analysis (black circle), from previous CMS results in $t \bar{t}$ events at $\sqrt{s}=13 \mathrm{TeV}$ for fully hadronic [10], dileptonic [14], and semileptonic [9] final states, and from ATLAS [7] and CMS $[8,22]$ analyses at $\sqrt{s}=8 \mathrm{TeV}$. The horizontal bars on the points show the combined statistical and systematic uncertainties in each measurement. The vertical dashed black line indicates the central value obtained from this measurement in the $l^{ \pm}$final state. The green band represents the combined statistical and profiled systematic uncertainties in the present result, whereas the yellow band shows the total uncertainty.

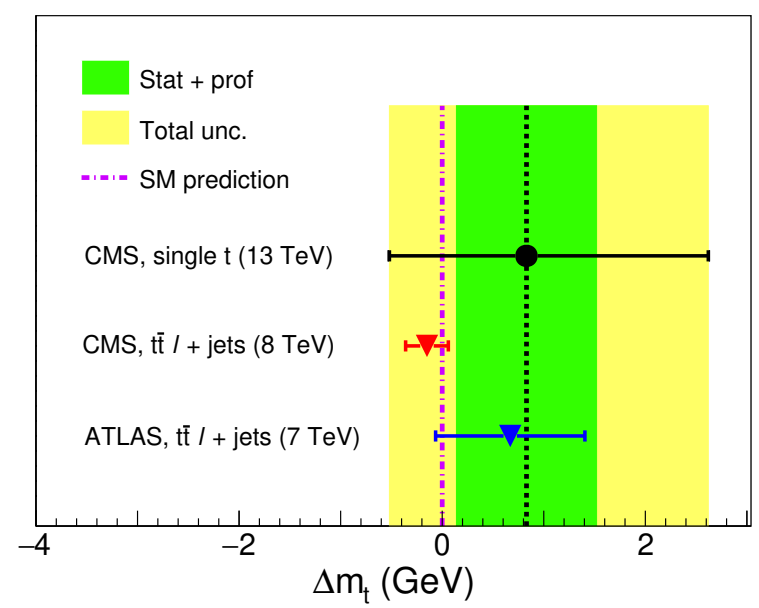

Figure 10. A comparison of the $\Delta m_{\mathrm{t}}$ measurement from this analysis (black circle) with the previous ATLAS [91] and CMS [92] results in $t \bar{t}$ events at 7 and $8 \mathrm{TeV}$, respectively. The horizontal bars on the points show the combined statistical and systematic uncertainties in each measurement. The vertical dashed black line indicates the central value obtained from this measurement, and the vertical dash-dotted magenta line is the SM prediction. The green band represents the combined statistical and profiled systematic uncertainties in the present result, whereas the yellow band shows the total uncertainty. 


\section{Summary}

Measurements of the top quark and antiquark masses, as well as their ratio and difference, are performed using a data sample enriched with single top quark events produced in proton-proton collisions at $\sqrt{s}=13 \mathrm{TeV}$. The analyzed data correspond to an integrated luminosity of $35.9 \mathrm{fb}^{-1}$ recorded by the CMS experiment at the LHC. Events containing an isolated muon or electron and two jets, of which one is b tagged, in the final state are used in the study. From the inclusive measurement the top quark mass is found to be $172.13_{-0.77}^{+0.76} \mathrm{GeV}$, where the uncertainty includes both the statistical and systematic components. The masses of the top quark and antiquark are separately determined as $172.62_{-0.75}^{+1.04}$ and $171.79_{-1.51}^{+1.44} \mathrm{GeV}$, respectively. These quantities are used to determine the mass ratio of the top antiquark to top quark of $0.9952_{-0.0104}^{+0.0079}$, along with the difference between the top quark and antiquark masses of $0.83_{-1.35}^{+1.79} \mathrm{GeV}$, both for the first time in single top quark production. The obtained mass ratio and difference agree with unity and zero, respectively, within the uncertainties, and are consistent with the conservation of CPT symmetry. This is the first measurement of the top quark mass in this particular final state to achieve a sub-GeV precision.

\section{Acknowledgments}

We congratulate our colleagues in the CERN accelerator departments for the excellent performance of the LHC and thank the technical and administrative staffs at CERN and at other CMS institutes for their contributions to the success of the CMS effort. In addition, we gratefully acknowledge the computing centers and personnel of the Worldwide LHC Computing Grid and other centers for delivering so effectively the computing infrastructure essential to our analyses. Finally, we acknowledge the enduring support for the construction and operation of the LHC, the CMS detector, and the supporting computing infrastructure provided by the following funding agencies: BMBWF and FWF (Austria); FNRS and FWO (Belgium); CNPq, CAPES, FAPERJ, FAPERGS, and FAPESP (Brazil); MES (Bulgaria); CERN; CAS, MoST, and NSFC (China); MINCIENCIAS (Colombia); MSES and CSF (Croatia); RIF (Cyprus); SENESCYT (Ecuador); MoER, ERC PUT and ERDF (Estonia); Academy of Finland, MEC, and HIP (Finland); CEA and CNRS/IN2P3 (France); BMBF, DFG, and HGF (Germany); GSRI (Greece); NKFIA (Hungary); DAE and DST (India); IPM (Iran); SFI (Ireland); INFN (Italy); MSIP and NRF (Republic of Korea); MES (Latvia); LAS (Lithuania); MOE and UM (Malaysia); BUAP, CINVESTAV, CONACYT, LNS, SEP, and UASLP-FAI (Mexico); MOS (Montenegro); MBIE (New Zealand); PAEC (Pakistan); MSHE and NSC (Poland); FCT (Portugal); JINR (Dubna); MON, RosAtom, RAS, RFBR, and NRC KI (Russia); MESTD (Serbia); SEIDI, CPAN, PCTI, and FEDER (Spain); MOSTR (Sri Lanka); Swiss Funding Agencies (Switzerland); MST (Taipei); ThEPCenter, IPST, STAR, and NSTDA (Thailand); TUBITAK and TAEK (Turkey); NASU (Ukraine); STFC (United Kingdom); DOE and NSF (U.S.A.).

Individuals have received support from the Marie-Curie program and the European Research Council and Horizon 2020 Grant, contract Nos. 675440, 724704, 752730, 758316, 
765710, 824093, 884104, and COST Action CA16108 (European Union); the Leventis Foundation; the Alfred P. Sloan Foundation; the Alexander von Humboldt Foundation; the Belgian Federal Science Policy Office; the Fonds pour la Formation à la Recherche dans l'Industrie et dans l'Agriculture (FRIA-Belgium); the Agentschap voor Innovatie door Wetenschap en Technologie (IWT-Belgium); the F.R.S.-FNRS and FWO (Belgium) under the "Excellence of Science - EOS" — be.h project n. 30820817; the Beijing Municipal Science \& Technology Commission, No. Z191100007219010; the Ministry of Education, Youth and Sports (MEYS) of the Czech Republic; the Deutsche Forschungsgemeinschaft (DFG), under Germany's Excellence Strategy - EXC 2121 "Quantum Universe" - 390833306, and under project number 400140256 — GRK2497; the Lendület ("Momentum") Program and the János Bolyai Research Scholarship of the Hungarian Academy of Sciences, the New National Excellence Program ÚNKP, the NKFIA research grants 123842, 123959, 124845, 124850, 125105, 128713, 128786, and 129058 (Hungary); the Council of Science and Industrial Research, India; the Latvian Council of Science; the Ministry of Science and Higher Education and the National Science Center, contracts Opus 2014/15/B/ST2/03998 and 2015/19/B/ST2/02861 (Poland); the Fundação para a Ciência e a Tecnologia, grant CEECIND/01334/2018 (Portugal); the National Priorities Research Program by Qatar National Research Fund; the Ministry of Science and Higher Education, project no. 14.W03.31.0026 (Russia); the Programa Estatal de Fomento de la Investigación Científica y Técnica de Excelencia María de Maeztu, grant MDM-2015-0509 and the Programa Severo Ochoa del Principado de Asturias; the Stavros Niarchos Foundation (Greece); the Rachadapisek Sompot Fund for Postdoctoral Fellowship, Chulalongkorn University and the Chulalongkorn Academic into Its 2nd Century Project Advancement Project (Thailand); the Kavli Foundation; the Nvidia Corporation; the SuperMicro Corporation; the Welch Foundation, contract C-1845; and the Weston Havens Foundation (U.S.A.).

\section{A MVA input variables and their correlations}

Figures 11 and 12 show the distributions of the other six BDT input variables in data and simulation for the muon and electron final states. Figure 13 presents the correlations among all BDT input variables for these final states before and after applying the decorrelation method available in TMVA. As expected, the correlations are significantly reduced after decorrelation. 

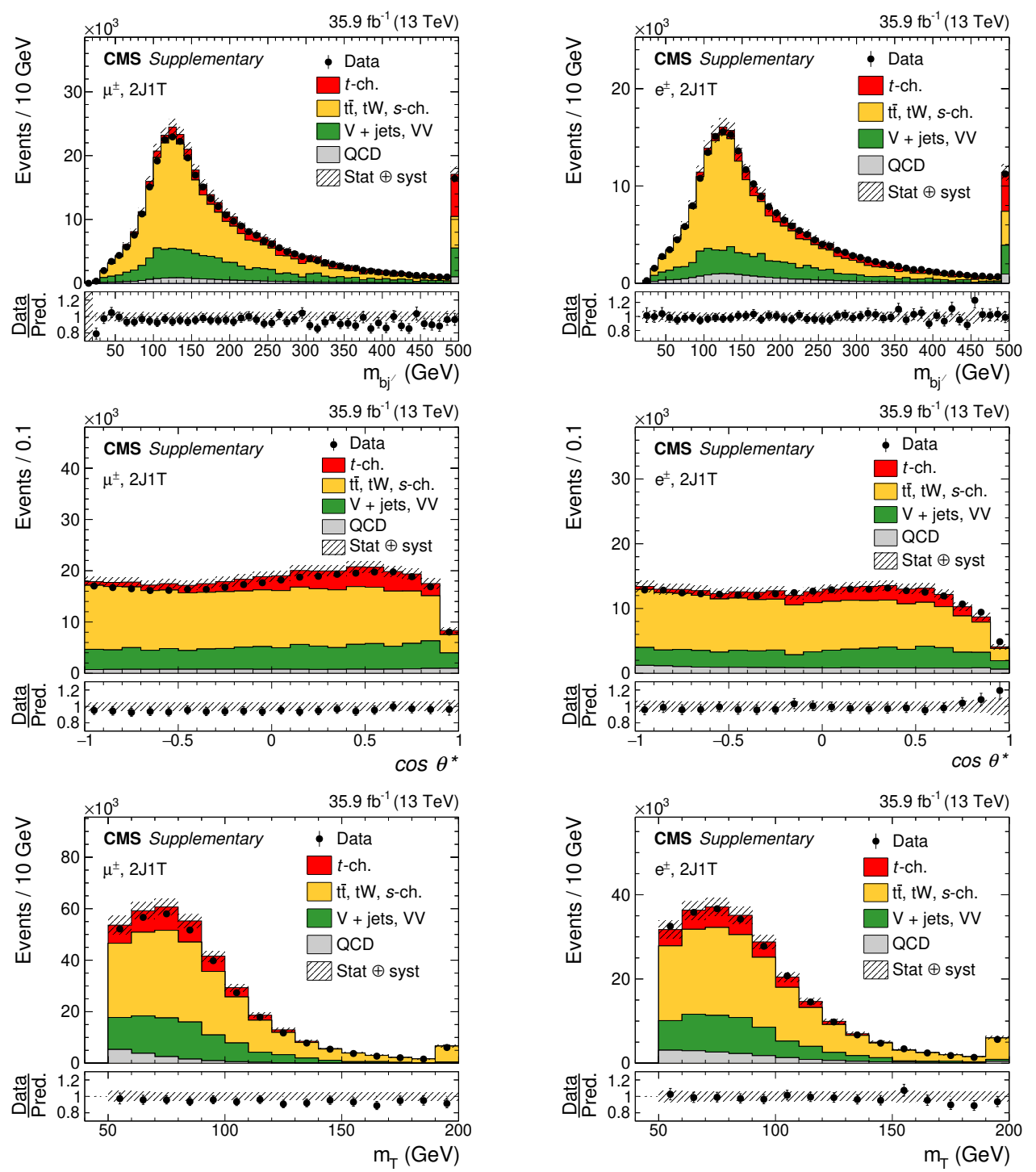

Figure 11. Distributions of $m_{\mathrm{bj}}$ (upper row), $\cos \theta^{*}$ (middle row), and $m_{\mathrm{T}}$ (lower row) for the muon (left) and electron (right) final states in the $2 \mathrm{~J} 1 \mathrm{~T}$ category for data (points) and simulation (colored histograms). The lower panel in each plot shows the ratio of the data to the predictions. The bands indicate the statistical and systematic uncertainties added in quadrature. The last bin in each of the upper- and lower-row plots includes the overflow. 

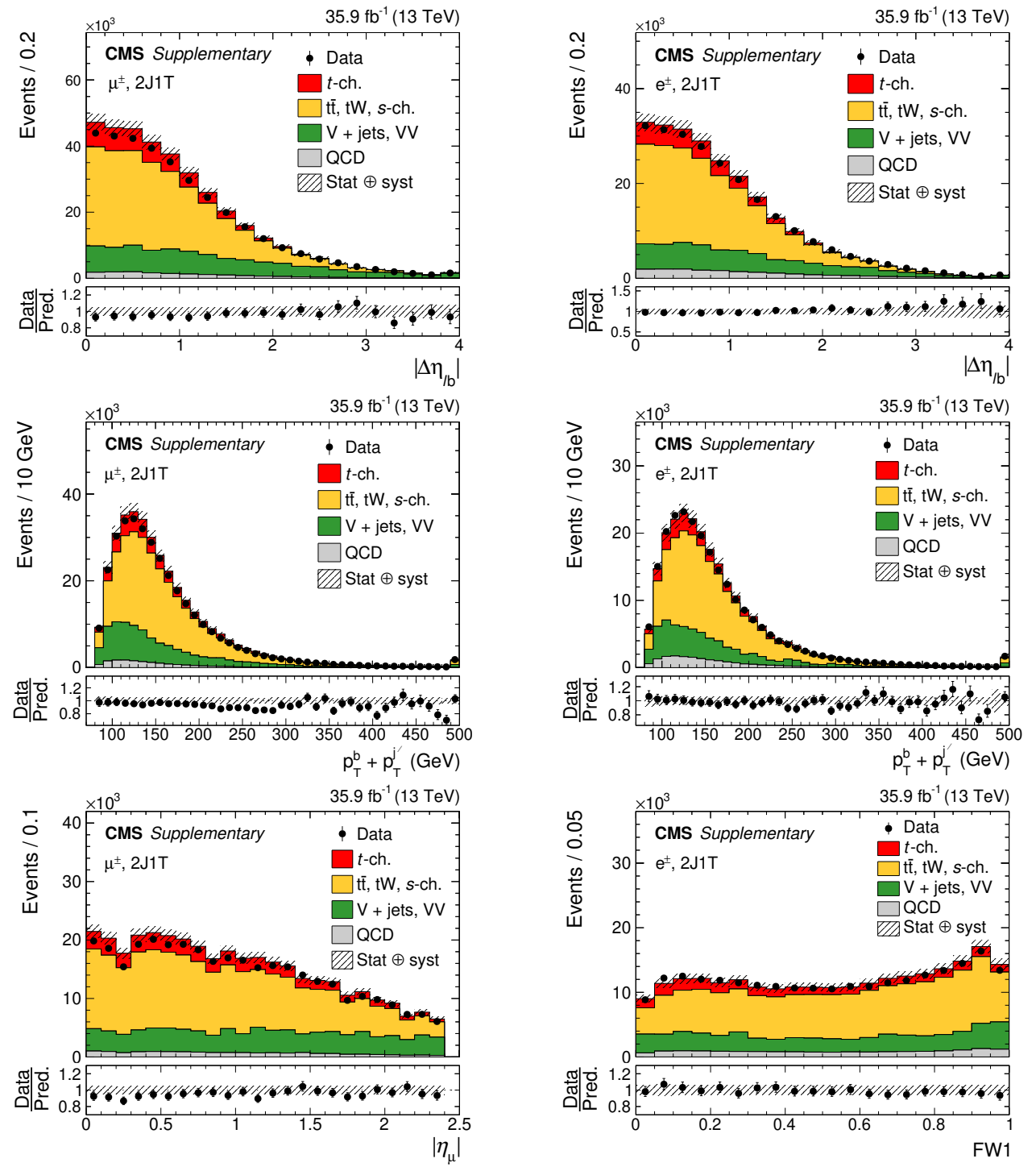

Figure 12. Distributions of $\left|\Delta \eta_{l \mathrm{~b}}\right|$ (upper row) and $p_{\mathrm{T}}^{\mathrm{b}}+p_{\mathrm{T}}^{\mathrm{j}^{\prime}}$ (middle row) for the muon (left) and electron (right) final states in the $2 \mathrm{~J} 1 \mathrm{~T}$ category for data (points) and simulation (colored histograms). The lower row shows the similar data-to-simulation comparison for $\left|\eta_{l}\right|$ (left) and FW1 (right) for the muon (left) and electron (right) final states in the 2J1T category. The lower panel in each plot shows the ratio of the data to the predictions. The bands indicate the statistical and systematic uncertainties added in quadrature. The last bin in each plot except for the lowerright one includes the overflow. 

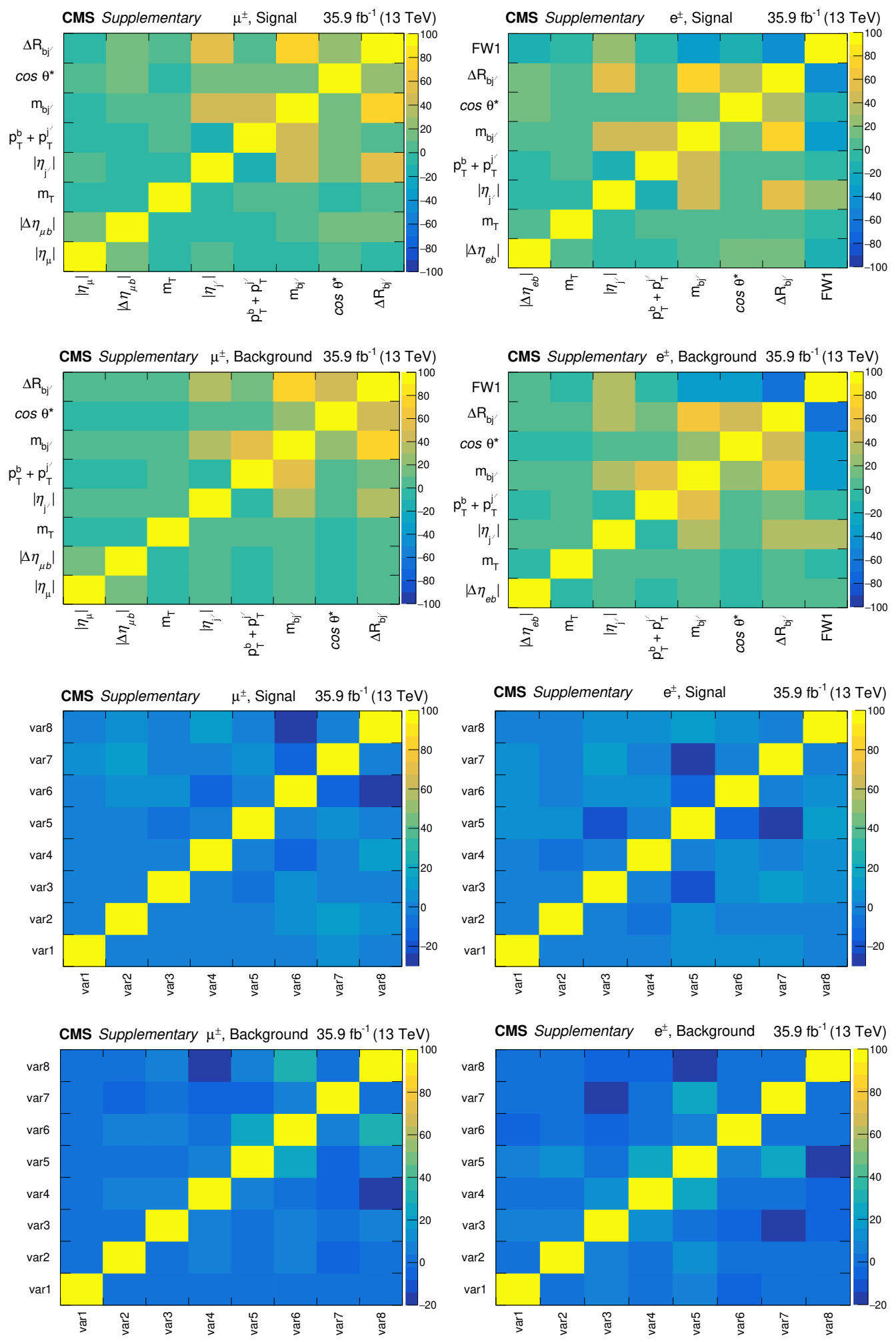

Figure 13. Correlations in \% among the BDT input variables used for the muon (left) and electron (right) final states in the signal and background events of the 2J1T category before (upper-two rows) and after (lower-two rows) decorrelation. 

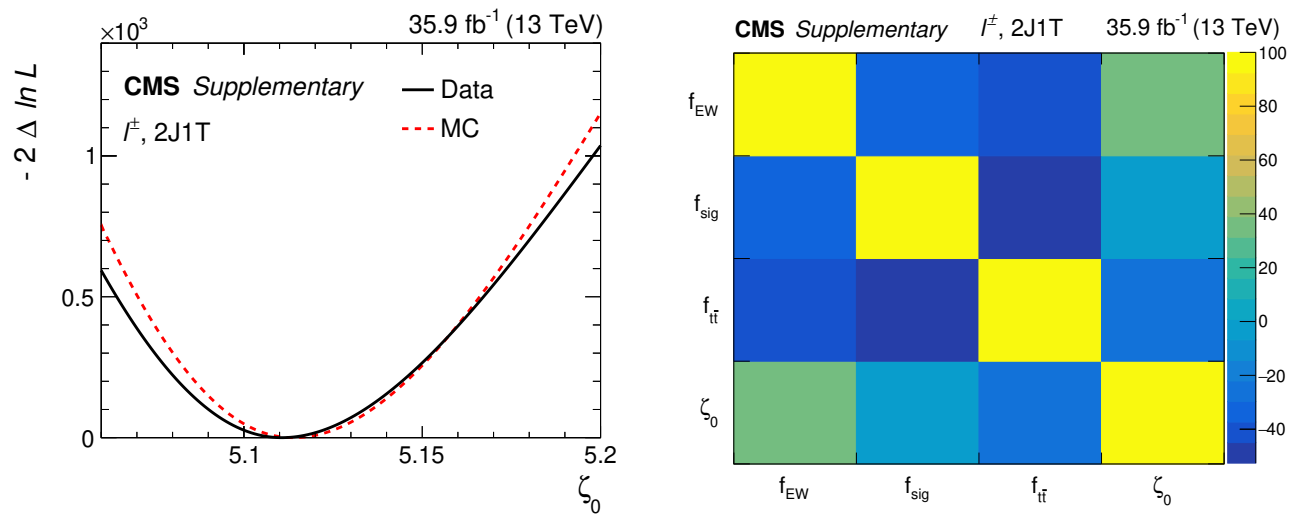

Figure 14. Scan of the profile likelihood ratio as a function of the POI for the parametric fit model used in the $l^{ \pm}$final state of the 2J1T category in data and simulated events (left). Correlations in $\%$ among the POI and nuisance parameters corresponding to the fit to data for the $l^{ \pm}$final state of the 2J1T category (right).

\section{B Likelihood scan and correlations among fit parameters}

Figure 14 (left) shows the scan of the profile likelihood ratio as a function of the POI for the ML fit model used to determine the $m_{\mathrm{t}}$ value in the charge-inclusive muon and electron final states. The scan results are presented both for data and simulation. Figure 14 (right) presents correlations among the POI and three nuisance parameters corresponding to the ML fit applied to data in the same final states.

Open Access. This article is distributed under the terms of the Creative Commons Attribution License (CC-BY 4.0), which permits any use, distribution and reproduction in any medium, provided the original author(s) and source are credited.

\section{References}

[1] J. Haller, A. Hoecker, R. Kogler, K. Mönig, T. Peiffer and J. Stelzer, Update of the global electroweak fit and constraints on two-Higgs-doublet models, Eur. Phys. J. C 78 (2018) 675 [arXiv: 1803.01853] [INSPIRE].

[2] G. Durieux et al., The electro-weak couplings of the top and bottom quarks - global fit and future prospects, JHEP 12 (2019) 098 [Erratum ibid. 01 (2021) 195] [arXiv:1907.10619] [INSPIRE].

[3] S. Alekhin, A. Djouadi and S. Moch, The top quark and Higgs boson masses and the stability of the electroweak vacuum, Phys. Lett. B 716 (2012) 214 [arXiv:1207.0980] [INSPIRE].

[4] G. Degrassi et al., Higgs mass and vacuum stability in the Standard Model at NNLO, JHEP 08 (2012) 098 [arXiv: 1205.6497] [INSPIRE].

[5] CMS collaboration, Identification of heavy-flavour jets with the CMS detector in pp collisions at $13 \mathrm{TeV}, 2018$ JINST 13 P05011 [arXiv:1712.07158] [INSPIRE].

[6] ATLAS collaboration, Performance of top-quark and W-boson tagging with ATLAS in run 2 of the LHC, Eur. Phys. J. C 79 (2019) 375 [arXiv: 1808.07858] [InSPIRE]. 
[7] ATLAS collaboration, Measurement of the top quark mass in the $t \bar{t} \rightarrow$ lepton + jets channel from $\sqrt{s}=8 \mathrm{TeV}$ ATLAS data and combination with previous results, Eur. Phys. J. C $\mathbf{7 9}$ (2019) 290 [arXiv: 1810.01772] [INSPIRE].

[8] CMS collaboration, Measurement of the top quark mass using proton-proton data at $\sqrt{s}=7$ and 8 TeV, Phys. Rev. D 93 (2016) 072004 [arXiv: 1509.04044] [InSPIRE].

[9] CMS collaboration, Measurement of the top quark mass with lepton+jets final states using pp collisions at $\sqrt{s}=13 \mathrm{TeV}$, Eur. Phys. J. C 78 (2018) 891 [arXiv:1805.01428] [InSPIRE].

[10] CMS collaboration, Measurement of the top quark mass in the all-jets final state at $\sqrt{s}=13 \mathrm{TeV}$ and combination with the lepton+jets channel, Eur. Phys. J. C 79 (2019) 313 [arXiv: 1812.10534] [INSPIRE].

[11] CMS collaboration, Measurement of the jet mass distribution and top quark mass in hadronic decays of boosted top quarks in pp collisions at $\sqrt{s}=13$ TeV, Phys. Rev. Lett. 124 (2020) 202001 [arXiv: 1911.03800] [InSPIRE].

[12] CMS collaboration, Measurement of $t \bar{t}$ normalised multi-differential cross sections in $p p$ collisions at $\sqrt{s}=13 \mathrm{TeV}$, and simultaneous determination of the strong coupling strength, top quark pole mass, and parton distribution functions, Eur. Phys. J. C 80 (2020) 658 [arXiv: 1904.05237] [INSPIRE].

[13] CMS collaboration, Running of the top quark mass from proton-proton collisions at $\sqrt{s}=13 \mathrm{TeV}$, Phys. Lett. B 803 (2020) 135263 [arXiv: 1909.09193] [INSPIRE].

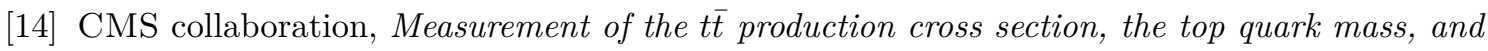
the strong coupling constant using dilepton events in pp collisions at $\sqrt{s}=13 \mathrm{TeV}$, Eur. Phys. J. C 79 (2019) 368 [arXiv:1812.10505] [INSPIRE].

[15] J.R. Christiansen and P.Z. Skands, String formation beyond leading colour, JHEP 08 (2015) 003 [arXiv: 1505.01681] [INSPIRE].

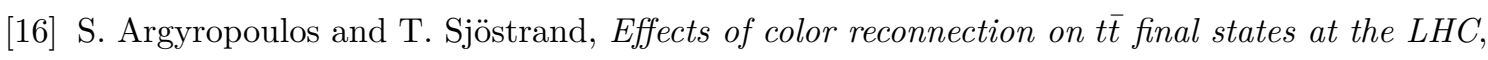
JHEP 11 (2014) 043 [arXiv: 1407.6653] [INSPIRE].

[17] M. Aliev, H. Lacker, U. Langenfeld, S. Moch, P. Uwer and M. Wiedermann, HATHOR: HAdronic Top and Heavy quarks crOss section calculatoR, Comput. Phys. Commun. 182 (2011) 1034 [arXiv: 1007.1327] [inSPIRE].

[18] P. Kant et al., HatHor for single top-quark production: Updated predictions and uncertainty estimates for single top-quark production in hadronic collisions, Comput. Phys. Commun. 191 (2015) 74 [arXiv: 1406.4403] [INSPIRE].

[19] CMS collaboration, Measurement of the single top quark and antiquark production cross sections in the $t$ channel and their ratio in proton-proton collisions at $\sqrt{s}=13 \mathrm{TeV}$, Phys. Lett. B 800 (2020) 135042 [arXiv: 1812.10514] [INSPIRE].

[20] M. Butenschoen, B. Dehnadi, A.H. Hoang, V. Mateu, M. Preisser and I.W. Stewart, Top quark mass calibration for Monte Carlo event generators, Phys. Rev. Lett. 117 (2016) 232001 [arXiv: 1608.01318] [INSPIRE].

[21] CMS collaboration, Precision luminosity measurement in proton-proton collisions at $\sqrt{s}=13 \mathrm{TeV}$ in 2015 and 2016 at CMS, Eur. Phys. J. C 81 (2021) 800 [arXiv:2104.01927] [INSPIRE]. 
[22] CMS collaboration, Measurement of the top quark mass using single top quark events in proton-proton collisions at $\sqrt{s}=8$ TeV, Eur. Phys. J. C 77 (2017) 354 [arXiv:1703.02530] [INSPIRE].

[23] O.W. Greenberg, CPT violation implies violation of Lorentz invariance, Phys. Rev. Lett. 89 (2002) 231602 [hep-ph/0201258] [INSPIRE].

[24] HEPData record for this analysis, CMS-TOP-19-009, (2021).

[25] CMS collaboration, The CMS experiment at the CERN LHC, 2008 JINST 3 S08004 [INSPIRE].

[26] CMS collaboration, The CMS trigger system, 2017 JINST 12 P01020 [arXiv:1609.02366] [INSPIRE].

[27] CMS collaboration, Performance of the CMS level-1 trigger in proton-proton collisions at $\sqrt{s}=13 \mathrm{TeV}, 2020 \mathrm{JINST} 15 \mathrm{P} 10017$ [arXiv:2006.10165] [INSPIRE].

[28] P. Nason, A new method for combining NLO QCD with shower Monte Carlo algorithms, JHEP 11 (2004) 040 [hep-ph/0409146] [INSPIRE].

[29] S. Frixione, P. Nason and C. Oleari, Matching NLO QCD computations with parton shower simulations: the POWHEG method, JHEP 11 (2007) 070 [arXiv:0709.2092] [INSPIRE].

[30] S. Alioli, P. Nason, C. Oleari and E. Re, A general framework for implementing NLO calculations in shower Monte Carlo programs: the POWHEG BOX, JHEP 06 (2010) 043 [arXiv:1002.2581] [INSPIRE].

[31] J. Alwall et al., The automated computation of tree-level and next-to-leading order differential cross sections, and their matching to parton shower simulations, JHEP 07 (2014) 079 [arXiv: 1405.0301] [INSPIRE].

[32] S. Alioli, P. Nason, C. Oleari and E. Re, NLO single-top production matched with shower in POWHEG: s-and t-channel contributions, JHEP 09 (2009) 111 [Erratum ibid. 02 (2010) 011] [arXiv:0907.4076] [INSPIRE].

[33] R. Frederix, E. Re and P. Torrielli, Single-top t-channel hadroproduction in the four-flavour scheme with POWHEG and aMC@NLO, JHEP 09 (2012) 130 [arXiv:1207.5391] [INSPIRE].

[34] ATLAS collaboration, Fiducial, total and differential cross-section measurements of t-channel single top-quark production in pp collisions at $8 \mathrm{TeV}$ using data collected by the ATLAS detector, Eur. Phys. J. C 77 (2017) 531 [arXiv: 1702.02859] [INSPIRE].

[35] CMS collaboration, Measurement of differential cross sections and charge ratios for t-channel single top quark production in proton-proton collisions at $\sqrt{s}=13$ TeV, Eur. Phys. J. C 80 (2020) 370 [arXiv: 1907.08330] [INSPIRE].

[36] S. Alioli, S.-O. Moch and P. Uwer, Hadronic top-quark pair-production with one jet and parton showering, JHEP 01 (2012) 137 [arXiv:1110.5251] [INSPIRE].

[37] M. Czakon and A. Mitov, Top++: a program for the calculation of the top-pair cross-section at hadron colliders, Comput. Phys. Commun. 185 (2014) 2930 [arXiv:1112.5675] [INSPIRE].

[38] E. Re, Single-top Wt-channel production matched with parton showers using the POWHEG method, Eur. Phys. J. C 71 (2011) 1547 [arXiv:1009.2450] [InSPIRE].

[39] N. Kidonakis, Two-loop soft anomalous dimensions for single top quark associated production with a $W^{-}$or $H^{-}$, Phys. Rev. D 82 (2010) 054018 [arXiv: 1005.4451] [InSPIRE]. 
[40] N. Kidonakis, Top quark production, in Helmholtz international summer school on physics of heavy quarks and hadrons, DESY-PROC-2013-03, (2014), pg. 139 [arXiv:1311.0283] [INSPIRE].

[41] R. Frederix and S. Frixione, Merging meets matching in MC@NLO, JHEP 12 (2012) 061 [arXiv: 1209.6215] [INSPIRE].

[42] R. Gavin, Y. Li, F. Petriello and S. Quackenbush, FEWZ 2.0: a code for hadronic Z production at next-to-next-to-leading order, Comput. Phys. Commun. 182 (2011) 2388 [arXiv: 1011.3540] [INSPIRE].

[43] R. Gavin, Y. Li, F. Petriello and S. Quackenbush, W physics at the LHC with FEWZ 2.1, Comput. Phys. Commun. 184 (2013) 208 [arXiv:1201.5896] [INSPIRE].

[44] Y. Li and F. Petriello, Combining QCD and electroweak corrections to dilepton production in FEWZ, Phys. Rev. D 86 (2012) 094034 [arXiv:1208.5967] [INSPIRE].

[45] T. Sjöstrand et al., An introduction to PYTHIA 8.2, Comput. Phys. Commun. 191 (2015) 159 [arXiv: 1410.3012] [INSPIRE].

[46] CMS collaboration, Event generator tunes obtained from underlying event and multiparton scattering measurements, Eur. Phys. J. C 76 (2016) 155 [arXiv:1512.00815] [INSPIRE].

[47] CMS collaboration, Investigations of the impact of the parton shower tuning in PYTHIA 8 in the modelling of $t \bar{t}$ at $\sqrt{s}=8$ and $13 \mathrm{TeV}$, Tech. Rep. CMS-PAS-TOP-16-021, CERN, Geneva, Switzerland (2016).

[48] NNPDF collaboration, Parton distributions for the LHC run II, JHEP 04 (2015) 040 [arXiv: 1410.8849] [INSPIRE].

[49] GEANT4 collaboration, GEANT4 - a simulation toolkit, Nucl. Instrum. Meth. A 506 (2003) 250 [INSPIRE].

[50] N. Kidonakis, Differential and total cross sections for top pair and single top production, in $20^{\text {th }}$ International workshop on deep-inelastic scattering and related subjects, DESY-PROC-2012-02, (2012), pg. 831 [arXiv:1205.3453] [INSPIRE].

[51] CMS collaboration, Particle-flow reconstruction and global event description with the CMS detector, 2017 JINST 12 P10003 [arXiv:1706.04965] [INSPIRE].

[52] W. Adam, R. Frühwirth, A. Strandlie and T. Todorov, Reconstruction of electrons with the Gaussian sum filter in the CMS tracker at LHC, eConf C 0303241 (2003) TULT009 [J. Phys. G 31 (2005) N9] [INSPIRE].

[53] CMS collaboration, Electron and photon reconstruction and identification with the CMS experiment at the CERN LHC, 2021 JINST 16 P05014 [arXiv:2012.06888] [INSPIRE].

[54] CMS collaboration, Performance of the CMS muon detector and muon reconstruction with proton-proton collisions at $\sqrt{s}=13 \mathrm{TeV}, 2018$ JINST $13 \mathrm{P} 06015$ [arXiv:1804.04528] [INSPIRE].

[55] M. Cacciari, G.P. Salam and G. Soyez, The anti- $k_{t}$ jet clustering algorithm, JHEP 04 (2008) 063 [arXiv:0802.1189] [INSPIRE].

[56] M. Cacciari, G.P. Salam and G. Soyez, FastJet user manual, Eur. Phys. J. C 72 (2012) 1896 [arXiv:1111.6097] [INSPIRE].

[57] CMS collaboration, Jet algorithms performance in $13 \mathrm{TeV}$ data, Tech. Rep. CMS-PAS-JME-16-003, CERN, Geneva, Switzerland (2017). 
[58] M. Cacciari and G.P. Salam, Pileup subtraction using jet areas, Phys. Lett. B 659 (2008) 119 [arXiv: 0707.1378] [INSPIRE].

[59] CMS collaboration, Jet energy scale and resolution in the CMS experiment in pp collisions at $8 \mathrm{TeV}, 2017$ JINST 12 P02014 [arXiv:1607.03663] [INSPIRE].

[60] CMS collaboration, Performance of missing transverse momentum reconstruction in proton-proton collisions at $\sqrt{s}=13 \mathrm{TeV}$ using the CMS detector, 2019 JINST $14 \mathrm{P} 07004$ [arXiv: 1903.06078] [INSPIRE].

[61] Particle Data Group collaboration, Review of particle physics, PTEP 2020 (2020) 083C01 [INSPIRE].

[62] CDF collaboration, First observation of electroweak single top quark production, Phys. Rev. Lett. 103 (2009) 092002 [arXiv: 0903.0885] [inSPIRE].

[63] D0 collaboration, Observation of single top quark production, Phys. Rev. Lett. 103 (2009) 092001 [arXiv: 0903.0850] [INSPIRE].

[64] A. Höcker et al., TMVA - toolkit for multivariate data analysis, physics/0703039 [INSPIRE].

[65] G.C. Fox and S. Wolfram, Event shapes in $e^{+} e^{-}$annihilation, Nucl. Phys. B 149 (1979) 413 [Erratum ibid. 157 (1979) 543] [INSPIRE].

[66] C. Bernaciak, M.S.A. Buschmann, A. Butter and T. Plehn, Fox-Wolfram moments in Higgs physics, Phys. Rev. D 87 (2013) 073014 [arXiv:1212.4436] [INSPIRE].

[67] Y. Freund and R.E. Schapire, A decision-theoretic generalization of on-line learning and an application to boosting, J. Comput. Syst. Sci. 55 (1997) 119.

[68] Belle collaboration, Evidence for the decay $B^{0} \rightarrow K^{+} K^{-} \pi^{0}$, Phys. Rev. D 87 (2013) 091101 [arXiv: 1304 .5312] [INSPIRE].

[69] W. Verkerke and D.P. Kirkby, The RooFit toolkit for data modeling, eConf C 0303241 (2003) MOLT007 [physics/0306116] [INSPIRE].

[70] H. Fanchiotti, C.A. Garcia Canal and M. Marucho, The Landau distribution, Int. J. Mod. Phys. C 17 (2006) 1461 [hep-ph/0305310] [INSPIRE].

[71] M.J. Oreglia, A study of the reactions $\psi^{\prime} \rightarrow \gamma \gamma \psi$, Ph.D. thesis, SLAC report SLAC-R-236, Stanford University, Stanford, CA, U.S.A. (1980).

[72] BeLLE collaboration, A detailed test of the CsI(Tl) calorimeter for BELLE with photon beams of energy between $20 \mathrm{MeV}$ and $5.4 \mathrm{GeV}$, Nucl. Instrum. Meth. A 441 (2000) 401 [INSPIRE].

[73] M. Czakon, P. Fiedler and A. Mitov, Total top-quark pair-production cross section at hadron colliders through $O\left(\alpha_{S}^{4}\right)$, Phys. Rev. Lett. 110 (2013) 252004 [arXiv:1303.6254] [INSPIRE].

[74] CMS collaboration, Measurement of the differential cross sections for the associated production of a $W$ boson and jets in proton-proton collisions at $\sqrt{s}=13 \mathrm{TeV}$, Phys. Rev. D 96 (2017) 072005 [arXiv: 1707.05979] [INSPIRE].

[75] CMS collaboration, Measurement of differential cross sections for $Z$ boson production in association with jets in proton-proton collisions at $\sqrt{s}=13$ TeV, Eur. Phys. J. C 78 (2018) 965 [arXiv: 1804.05252] [INSPIRE].

[76] CMS collaboration, Determination of jet energy calibration and transverse momentum resolution in CMS, 2011 JINST 6 P11002 [arXiv:1107.4277] [INSPIRE]. 
[77] CMS and ATLAS collaborations, Jet energy scale uncertainty correlations between ATLAS and CMS at $8 \mathrm{TeV}$, Tech. Rep. CMS-PAS-JME-15-001, CERN, Geneva, Switzerland (2015) [ATL-PHYS-PUB-2015-049].

[78] CMS collaboration, Performance of the CMS missing transverse momentum reconstruction in pp data at $\sqrt{s}=8 \mathrm{TeV}, 2015$ JINST 10 P02006 [arXiv: 1411.0511] [INSPIRE].

[79] CMS collaboration, Measurements of inclusive $W$ and $Z$ cross sections in pp collisions at $\sqrt{s}=7 \mathrm{TeV}$, JHEP 01 (2011) 080 [arXiv:1012.2466] [INSPIRE].

[80] CMS collaboration, Measurement of the inelastic proton-proton cross section at $\sqrt{s}=13 \mathrm{TeV}$, JHEP 07 (2018) 161 [arXiv: 1802.02613] [INSPIRE].

[81] CMS collaboration, CMS luminosity measurements for the 2016 data taking period, Tech. Rep. CMS-PAS-LUM-17-001, CERN, Geneva, Switzerland (2017).

[82] CMS collaboration, Study of the underlying event in top quark pair production in pp collisions at $13 \mathrm{TeV}$, Eur. Phys. J. C 79 (2019) 123 [arXiv:1807.02810] [inSPIRE].

[83] M. Bähr et al., HERWIG++ physics and manual, Eur. Phys. J. C 58 (2008) 639 [arXiv: 0803.0883] [INSPIRE].

[84] ALEPH collaboration, Study of the fragmentation of $b$ quarks into $B$ mesons at the $Z$ peak, Phys. Lett. B 512 (2001) 30 [hep-ex/0106051] [INSPIRE].

[85] DELPHI collaboration, A study of the b-quark fragmentation function with the DELPHI detector at LEP I and an averaged distribution obtained at the $Z$ pole, Eur. Phys. J. C 71 (2011) 1557 [arXiv: 1102.4748] [INSPIRE].

[86] SLD collaboration, Measurement of the $b$ quark fragmentation function in $Z^{0}$ decays, Phys. Rev. D 65 (2002) 092006 [Erratum ibid. 66 (2002) 079905] [hep-ex/0202031] [INSPIRE].

[87] M.G. Bowler, $e^{+} e^{-}$production of heavy quarks in the string model, Z. Phys. C 11 (1981) 169 [INSPIRE].

[88] C. Peterson, D. Schlatter, I. Schmitt and P.M. Zerwas, Scaling violations in inclusive $e^{+} e^{-}$ annihilation spectra, Phys. Rev. D 27 (1983) 105 [INSPIRE].

[89] A. Kalogeropoulos and J. Alwall, The SysCalc code: a tool to derive theoretical systematic uncertainties, arXiv:1801.08401 [INSPIRE].

[90] CMS collaboration, Measurement of the differential cross section for top quark pair production in pp collisions at $\sqrt{s}=8 \mathrm{TeV}$, Eur. Phys. J. C 75 (2015) 542 [arXiv: 1505.04480] [INSPIRE].

[91] ATLAS collaboration, Measurement of the mass difference between top and anti-top quarks in pp collisions at $\sqrt{s}=7 \mathrm{TeV}$ using the ATLAS detector, Phys. Lett. B 728 (2014) 363 [arXiv: 1310.6527] [INSPIRE].

[92] CMS collaboration, Measurement of the mass difference between top quark and antiquark in pp collisions at $\sqrt{s}=8 \mathrm{TeV}$, Phys. Lett. $B \mathbf{7 7 0}$ (2017) 50 [arXiv:1610.09551] [INSPIRE]. 


\section{The CMS collaboration}

\section{Yerevan Physics Institute, Yerevan, Armenia}

A. Tumasyan

\section{Institut für Hochenergiephysik, Wien, Austria}

W. Adam, J.W. Andrejkovic, T. Bergauer, S. Chatterjee, M. Dragicevic, A. Es-

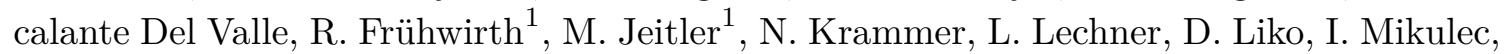
P. Paulitsch, F.M. Pitters, J. Schieck ${ }^{1}$, R. Schöfbeck, M. Spanring, S. Templ, W. Waltenberger, C.-E. Wulz ${ }^{1}$

Institute for Nuclear Problems, Minsk, Belarus

V. Chekhovsky, A. Litomin, V. Makarenko

\section{Universiteit Antwerpen, Antwerpen, Belgium}

M.R. Darwish ${ }^{2}$, E.A. De Wolf, X. Janssen, T. Kello ${ }^{3}$, A. Lelek, H. Rejeb Sfar, P. Van Mechelen, S. Van Putte, N. Van Remortel

\section{Vrije Universiteit Brussel, Brussel, Belgium}

F. Blekman, E.S. Bols, J. D'Hondt, J. De Clercq, M. Delcourt, H. El Faham, S. Lowette, S. Moortgat, A. Morton, D. Müller, A.R. Sahasransu, S. Tavernier, W. Van Doninck, P. Van Mulders

\section{Université Libre de Bruxelles, Bruxelles, Belgium}

D. Beghin, B. Bilin, B. Clerbaux, G. De Lentdecker, L. Favart, A. Grebenyuk, A.K. Kalsi, K. Lee, M. Mahdavikhorrami, I. Makarenko, L. Moureaux, L. Pétré, A. Popov, N. Postiau, E. Starling, L. Thomas, M. Vanden Bemden, C. Vander Velde, P. Vanlaer, D. Vannerom, L. Wezenbeek

\section{Ghent University, Ghent, Belgium}

T. Cornelis, D. Dobur, J. Knolle, L. Lambrecht, G. Mestdach, M. Niedziela, C. Roskas, A. Samalan, K. Skovpen, M. Tytgat, W. Verbeke, B. Vermassen, M. Vit

\section{Université Catholique de Louvain, Louvain-la-Neuve, Belgium}

A. Bethani, G. Bruno, F. Bury, C. Caputo, P. David, C. Delaere, I.S. Donertas, A. Giammanco, K. Jaffel, Sa. Jain, V. Lemaitre, K. Mondal, J. Prisciandaro, A. Taliercio, M. Teklishyn, T.T. Tran, P. Vischia, S. Wertz

\section{Centro Brasileiro de Pesquisas Fisicas, Rio de Janeiro, Brazil}

G.A. Alves, C. Hensel, A. Moraes

\section{Universidade do Estado do Rio de Janeiro, Rio de Janeiro, Brazil}

W.L. Aldá Júnior, M. Alves Gallo Pereira, M. Barroso Ferreira Filho, H. BRANDAO MALBOUISSON, W. Carvalho, J. Chinellato ${ }^{4}$, E.M. Da Costa, G.G. Da Silveira ${ }^{5}$, D. De Jesus Damiao, S. Fonseca De Souza, D. Matos Figueiredo, C. Mora Herrera, K. Mota Amarilo, L. Mundim, H. Nogima, P. Rebello Teles, A. Santoro, S.M. Silva Do Amaral, A. Sznajder, M. Thiel, F. Torres Da Silva De Araujo, A. Vilela Pereira 
Universidade Estadual Paulista ${ }^{a}$, Universidade Federal do $\mathrm{ABC}^{b}$, São Paulo, Brazil

C.A. Bernardes ${ }^{a, a, 5}$, L. Calligaris ${ }^{a}$, T.R. Fernandez Perez Tomei ${ }^{a}$, E.M. Gregores ${ }^{a, b}$, D.S. Lemos ${ }^{a}$, P.G. Mercadante ${ }^{a, b}$, S.F. Novaes ${ }^{a}$, Sandra S. Padula ${ }^{a}$

Institute for Nuclear Research and Nuclear Energy, Bulgarian Academy of Sciences, Sofia, Bulgaria

A. Aleksandrov, G. Antchev, R. Hadjiiska, P. Iaydjiev, M. Misheva, M. Rodozov,

M. Shopova, G. Sultanov

University of Sofia, Sofia, Bulgaria

A. Dimitrov, T. Ivanov, L. Litov, B. Pavlov, P. Petkov, A. Petrov

Beihang University, Beijing, China

T. Cheng, Q. Guo, T. Javaid ${ }^{6}$, M. Mittal, H. Wang, L. Yuan

Department of Physics, Tsinghua University

M. Ahmad, G. Bauer, C. Dozen ${ }^{7}$, Z. Hu, J. Martins ${ }^{8}$, Y. Wang, K. Yi ${ }^{9,10}$

Institute of High Energy Physics, Beijing, China

E. Chapon, G.M. Chen ${ }^{6}$, H.S. Chen ${ }^{6}$, M. Chen, F. Iemmi, A. Kapoor, D. Leggat, H. Liao, Z.-A. LIU ${ }^{6}$, V. Milosevic, F. Monti, R. Sharma, J. Tao, J. Thomas-wilsker, J. Wang, H. Zhang, S. Zhang ${ }^{6}$, J. Zhao

State Key Laboratory of Nuclear Physics and Technology, Peking University, Beijing, China

A. Agapitos, Y. An, Y. Ban, C. Chen, A. Levin, Q. Li, X. Lyu, Y. Mao, S.J. Qian, D. Wang, Q. Wang, J. Xiao

\section{Sun Yat-Sen University, Guangzhou, China}

M. Lu, Z. You

Institute of Modern Physics and Key Laboratory of Nuclear Physics and Ionbeam Application (MOE) — Fudan University, Shanghai, China

X. $\mathrm{Gao}^{3}, \mathrm{H}$. Okawa

Zhejiang University, Hangzhou, China

Z. Lin, M. Xiao

Universidad de Los Andes, Bogota, Colombia

C. Avila, A. Cabrera, C. Florez, J. Fraga, A. Sarkar, M.A. Segura Delgado

Universidad de Antioquia, Medellin, Colombia

J. Mejia Guisao, F. Ramirez, J.D. Ruiz Alvarez, C.A. Salazar González

University of Split, Faculty of Electrical Engineering, Mechanical Engineering and Naval Architecture, Split, Croatia

D. Giljanovic, N. Godinovic, D. Lelas, I. Puljak 
University of Split, Faculty of Science, Split, Croatia

Z. Antunovic, M. Kovac, T. Sculac

Institute Rudjer Boskovic, Zagreb, Croatia

V. Brigljevic, D. Ferencek, D. Majumder, M. Roguljic, A. Starodumov ${ }^{11}$, T. Susa

University of Cyprus, Nicosia, Cyprus

A. Attikis, K. Christoforou, E. Erodotou, A. Ioannou, G. Kole, M. Kolosova, S. Konstantinou, J. Mousa, C. Nicolaou, F. Ptochos, P.A. Razis, H. Rykaczewski, H. Saka

Charles University, Prague, Czech Republic

M. Finger ${ }^{12}$, M. Finger Jr. ${ }^{12}$, A. Kveton

Escuela Politecnica Nacional, Quito, Ecuador

E. Ayala

Universidad San Francisco de Quito, Quito, Ecuador

E. Carrera Jarrin

Academy of Scientific Research and Technology of the Arab Republic of Egypt, Egyptian Network of High Energy Physics, Cairo, Egypt

S. Abu Zeid ${ }^{13}$, S. Elgammal ${ }^{14}$

Center for High Energy Physics (CHEP-FU), Fayoum University, El-Fayoum, Egypt

A. Lotfy, M.A. Mahmoud

National Institute of Chemical Physics and Biophysics, Tallinn, Estonia

S. Bhowmik, R.K. Dewanjee, K. Ehataht, M. Kadastik, S. Nandan, C. Nielsen, J. Pata, M. Raidal, L. Tani, C. Veelken

Department of Physics, University of Helsinki, Helsinki, Finland

P. Eerola, L. Forthomme, H. Kirschenmann, K. Osterberg, M. Voutilainen

Helsinki Institute of Physics, Helsinki, Finland

S. Bharthuar, E. Brücken, F. Garcia, J. Havukainen, M.S. Kim, R. Kinnunen, T. Lampén, K. Lassila-Perini, S. Lehti, T. Lindén, M. Lotti, L. Martikainen, M. Myllymäki, J. Ott, H. Siikonen, E. Tuominen, J. Tuominiemi

Lappeenranta University of Technology, Lappeenranta, Finland

P. Luukka, H. Petrow, T. Tuuva

IRFU, CEA, Université Paris-Saclay, Gif-sur-Yvette, France

C. Amendola, M. Besancon, F. Couderc, M. Dejardin, D. Denegri, J.L. Faure, F. Ferri, S. Ganjour, A. Givernaud, P. Gras, G. Hamel de Monchenault, P. Jarry, B. Lenzi, E. Locci, J. Malcles, J. Rander, A. Rosowsky, M.Ö. Sahin, A. Savoy-Navarro ${ }^{15}$, M. Titov, G.B. Yu

Laboratoire Leprince-Ringuet, CNRS/IN2P3, Ecole Polytechnique, Institut Polytechnique de Paris, Palaiseau, France

S. Ahuja, F. Beaudette, M. Bonanomi, A. Buchot Perraguin, P. Busson, A. Cappati, C. Charlot, O. Davignon, B. Diab, G. Falmagne, S. Ghosh, R. Granier de Cassagnac, 
A. Hakimi, I. Kucher, J. Motta, M. Nguyen, C. Ochando, P. Paganini, J. Rembser, R. Salerno, J.B. Sauvan, Y. Sirois, A. Tarabini, A. Zabi, A. Zghiche

\section{Université de Strasbourg, CNRS, IPHC UMR 7178, Strasbourg, France}

J.-L. Agram ${ }^{16}$, J. Andrea, D. Apparu, D. Bloch, G. Bourgatte, J.-M. Brom, E.C. Chabert, C. Collard, D. Darej, J.-C. Fontaine ${ }^{16}$, U. Goerlach, C. Grimault, A.-C. Le Bihan, E. Nibigira, P. Van Hove

\section{Institut de Physique des 2 Infinis de Lyon (IP2I ), Villeurbanne, France}

E. Asilar, S. Beauceron, C. Bernet, G. Boudoul, C. Camen, A. Carle, N. Chanon, D. Contardo, P. Depasse, H. El Mamouni, J. Fay, S. Gascon, M. Gouzevitch, B. Ille, I.B. Laktineh, H. Lattaud, A. Lesauvage, M. Lethuillier, L. Mirabito, S. Perries, K. Shchablo, V. Sordini, L. Torterotot, G. Touquet, M. Vander Donckt, S. Viret

\section{Georgian Technical University, Tbilisi, Georgia}

A. Khvedelidze ${ }^{12}$, I. Lomidze, Z. Tsamalaidze ${ }^{12}$

\section{RWTH Aachen University, I. Physikalisches Institut, Aachen, Germany}

L. Feld, K. Klein, M. Lipinski, D. Meuser, A. Pauls, M.P. Rauch, N. Röwert, J. Schulz, M. Teroerde

\section{RWTH Aachen University, III. Physikalisches Institut A, Aachen, Germany}

A. Dodonova, D. Eliseev, M. Erdmann, P. Fackeldey, B. Fischer, S. Ghosh, T. Hebbeker, K. Hoepfner, F. Ivone, H. Keller, L. Mastrolorenzo, M. Merschmeyer, A. Meyer, G. Mocellin, S. Mondal, S. Mukherjee, D. Noll, A. Novak, T. Pook, A. Pozdnyakov, Y. Rath, H. Reithler, J. Roemer, A. Schmidt, S.C. Schuler, A. Sharma, L. Vigilante, S. Wiedenbeck, S. Zaleski

\section{RWTH Aachen University, III. Physikalisches Institut B, Aachen, Germany}

C. Dziwok, G. Flügge, W. Haj Ahmad ${ }^{17}$, O. Hlushchenko, T. Kress, A. Nowack, C. Pistone, O. Pooth, D. Roy, H. Sert, A. Stahl ${ }^{18}$, T. Ziemons

\section{Deutsches Elektronen-Synchrotron, Hamburg, Germany}

H. Aarup Petersen, M. Aldaya Martin, P. Asmuss, I. Babounikau, S. Baxter, O. Behnke, A. Bermúdez Martínez, S. Bhattacharya, A.A. Bin Anuar, K. Borras ${ }^{19}$, V. Botta, D. Brunner, A. Campbell, A. Cardini, C. Cheng, F. Colombina, S. Consuegra Rodríguez, G. Correia Silva, V. Danilov, L. Didukh, G. Eckerlin, D. Eckstein, L.I. Estevez Banos, O. Filatov, E. Gallo ${ }^{20}$, A. Geiser, A. Giraldi, A. Grohsjean, M. Guthoff, A. Jafari ${ }^{21}$, N.Z. Jomhari, H. Jung, A. Kasem ${ }^{19}$, M. Kasemann, H. Kaveh, C. Kleinwort, D. Krücker, W. Lange, J. Lidrych, K. Lipka, W. Lohmann ${ }^{22}$, R. Mankel, I.-A. Melzer-Pellmann, M. Mendizabal Morentin, J. Metwally, A.B. Meyer, M. Meyer, J. Mnich, A. Mussgiller, Y. Otarid, D. Pérez Adán, D. Pitzl, A. Raspereza, B. Ribeiro Lopes, J. Rübenach, A. Saggio, A. Saibel, M. Savitskyi, M. Scham, V. Scheurer, P. Schütze, C. Schwanenberger ${ }^{20}$, A. Singh, R.E. Sosa Ricardo, D. Stafford, N. Tonon, O. Turkot, M. Van De Klundert, R. Walsh, D. Walter, Y. Wen, K. Wichmann, L. Wiens, C. Wissing, S. Wuchterl 


\section{University of Hamburg, Hamburg, Germany}

R. Aggleton, S. Albrecht, S. Bein, L. Benato, A. Benecke, P. Connor, K. De Leo, M. Eich, F. Feindt, A. Fröhlich, C. Garbers, E. Garutti, P. Gunnellini, J. Haller, A. Hinzmann, G. Kasieczka, R. Klanner, R. Kogler, T. Kramer, V. Kutzner, J. Lange, T. Lange, A. Lobanov, A. Malara, A. Nigamova, K.J. Pena Rodriguez, O. Rieger, P. Schleper, M. Schröder, J. Schwandt, D. Schwarz, J. Sonneveld, H. Stadie, G. Steinbrück, A. Tews, B. Vormwald, I. Zoi

\section{Karlsruher Institut fuer Technologie, Karlsruhe, Germany}

J. Bechtel, T. Berger, E. Butz, R. Caspart, T. Chwalek, W. De Boer ${ }^{\dagger}$, A. Dierlamm, A. Droll, K. El Morabit, N. Faltermann, M. Giffels, J.o. Gosewisch, A. Gottmann, F. Hartmann ${ }^{18}$, C. Heidecker, U. Husemann, I. Katkov ${ }^{23}$, P. Keicher, R. Koppenhöfer, S. Maier, M. Metzler, S. Mitra, Th. Müller, M. Neukum, A. Nürnberg, G. Quast, K. Rabbertz, J. Rauser, D. Savoiu, M. Schnepf, D. Seith, I. Shvetsov, H.J. Simonis, R. Ulrich, J. Van Der Linden, R.F. Von Cube, M. Wassmer, M. Weber, S. Wieland, R. Wolf, S. Wozniewski, S. Wunsch

Institute of Nuclear and Particle Physics (INPP), NCSR Demokritos, Aghia Paraskevi, Greece

G. Anagnostou, G. Daskalakis, T. Geralis, A. Kyriakis, D. Loukas, A. Stakia

\section{National and Kapodistrian University of Athens, Athens, Greece}

M. Diamantopoulou, D. Karasavvas, G. Karathanasis, P. Kontaxakis, C.K. Koraka, A. Manousakis-katsikakis, A. Panagiotou, I. Papavergou, N. Saoulidou, K. Theofilatos, E. Tziaferi, K. Vellidis, E. Vourliotis

National Technical University of Athens, Athens, Greece

G. Bakas, K. Kousouris, I. Papakrivopoulos, G. Tsipolitis, A. Zacharopoulou

University of Ioánnina, Ioánnina, Greece

I. Evangelou, C. Foudas, P. Gianneios, P. Katsoulis, P. Kokkas, N. Manthos, I. Papadopoulos, J. Strologas

MTA-ELTE Lendület CMS Particle and Nuclear Physics Group, Eötvös Loránd University

M. Csanad, K. Farkas, M.M.A. Gadallah ${ }^{24}$, S. Lökös ${ }^{25}$, P. Major, K. Mandal, A. Mehta, G. Pasztor, A.J. Rádl, O. Surányi, G.I. Veres

Wigner Research Centre for Physics, Budapest, Hungary

M. Bartók ${ }^{26}$, G. Bencze, C. Hajdu, D. Horvath ${ }^{27}$, F. Sikler, V. Veszpremi, G. Vesztergombi ${ }^{\dagger}$

Institute of Nuclear Research ATOMKI, Debrecen, Hungary

S. Czellar, J. Karancsi ${ }^{26}$, J. Molnar, Z. Szillasi, D. Teyssier

Institute of Physics, University of Debrecen

P. Raics, Z.L. Trocsanyi ${ }^{28}$, B. Ujvari

Karoly Robert Campus, MATE Institute of Technology

T. Csorgo ${ }^{29}$, F. Nemes ${ }^{29}$, T. Novak 
Indian Institute of Science (IISc), Bangalore, India

J.R. Komaragiri, D. Kumar, L. Panwar, P.C. Tiwari

National Institute of Science Education and Research, HBNI, Bhubaneswar, India

S. Bahinipati ${ }^{30}$, C. Kar, P. Mal, T. Mishra, V.K. Muraleedharan Nair Bindhu ${ }^{31}$, A. Nayak ${ }^{31}$, P. Saha, N. Sur, S.K. Swain, D. Vats ${ }^{31}$

Panjab University, Chandigarh, India

S. Bansal, S.B. Beri, V. Bhatnagar, G. Chaudhary, S. Chauhan, N. Dhingra ${ }^{32}$, R. Gupta, A. Kaur, M. Kaur, S. Kaur, P. Kumari, M. Meena, K. Sandeep, J.B. Singh, A.K. Virdi

University of Delhi, Delhi, India

A. Ahmed, A. Bhardwaj, B.C. Choudhary, M. Gola, S. Keshri, A. Kumar, M. Naimuddin, P. Priyanka, K. Ranjan, A. Shah

Saha Institute of Nuclear Physics, HBNI, Kolkata, India

M. Bharti ${ }^{33}$, R. Bhattacharya, S. Bhattacharya, D. Bhowmik, S. Dutta, S. Dutta, B. Gomber ${ }^{34}$, M. Maity ${ }^{35}$, P. Palit, P.K. Rout, G. Saha, B. Sahu, S. Sarkar, M. Sharan, B. Singh ${ }^{33}$, S. Thakur ${ }^{33}$

Indian Institute of Technology Madras, Madras, India

P.K. Behera, S.C. Behera, P. Kalbhor, A. Muhammad, R. Pradhan, P.R. Pujahari, A. Sharma, A.K. Sikdar

Bhabha Atomic Research Centre, Mumbai, India

D. Dutta, V. Jha, V. Kumar, D.K. Mishra, K. Naskar ${ }^{36}$, P.K. Netrakanti, L.M. Pant, P. Shukla

Tata Institute of Fundamental Research-A, Mumbai, India

T. Aziz, S. Dugad, R. Karnam, M. Kumar, G.B. Mohanty, U. Sarkar

Tata Institute of Fundamental Research-B, Mumbai, India

S. Banerjee, R. Chudasama, M. Guchait, S. Karmakar, S. Kumar, G. Majumder, K. Mazumdar, S. Mukherjee

Indian Institute of Science Education and Research (IISER), Pune, India

K. Alpana, S. Dube, B. Kansal, A. Laha, S. Pandey, A. Rane, A. Rastogi, S. Sharma

Department of Physics, Isfahan University of Technology

H. Bakhshiansohi ${ }^{37}$, M. Zeinali ${ }^{38}$

Institute for Research in Fundamental Sciences (IPM), Tehran, Iran

S. Chenarani ${ }^{39}$, S.M. Etesami, M. Khakzad, M. Mohammadi Najafabadi

University College Dublin, Dublin, Ireland

M. Grunewald 
INFN Sezione di Bari ${ }^{a}$, Università di Bari ${ }^{b}$, Politecnico di Bari ${ }^{c}$, Bari, Italy

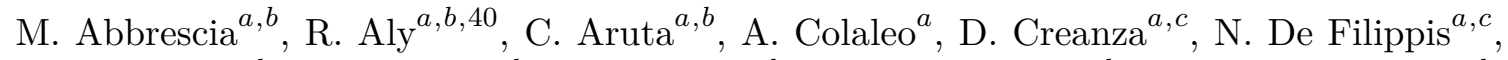
M. De Palma ${ }^{a, b}$, A. Di Florio ${ }^{a, b}$, A. Di Pilato ${ }^{a, b}$, W. Elmetenawee ${ }^{a, b}{\text {, L. } \text { Fiore }^{a} \text {, A. Gelmi }}^{a, b}$,

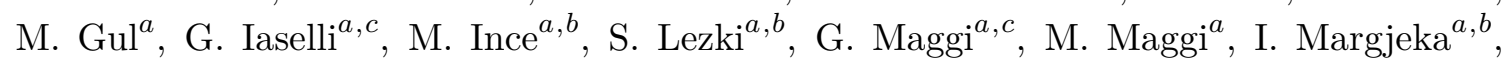
V. Mastrapasqua ${ }^{a, b}$, J.A. Merlin ${ }^{a}$, S. My ${ }^{a, b}$, S. Nuzzo ${ }^{a, b}$, A. Pellecchia ${ }^{a, b}$, A. Pompili ${ }^{a, b}$, G. Pugliese ${ }^{a, c}$, A. Ranieri ${ }^{a}$, G. Selvaggi ${ }^{a, b}$, L. Silvestris ${ }^{a}$, F.M. Simone ${ }^{a, b}$, R. Venditti ${ }^{a}$, P. Verwilligen ${ }^{a}$

INFN Sezione di Bologna ${ }^{a}$, Università di Bologna ${ }^{b}$, Bologna, Italy

G. Abbiendi ${ }^{a}$, C. Battilana ${ }^{a, b}$, D. Bonacorsi ${ }^{a, b}$, L. Borgonovi ${ }^{a}$, L. Brigliadori ${ }^{a}$, R. Campanini ${ }^{a, b}$, P. Capiluppi ${ }^{a, b}$, A. Castro ${ }^{a, b}$, F.R. Cavallo ${ }^{a}$, M. Cuffiani ${ }^{a, b}$, G.M. Dallavalle ${ }^{a}$, T. Diotalevi ${ }^{a, b}$, F. Fabbri ${ }^{a}$, A. Fanfani ${ }^{a, b}$, P. Giacomelli $^{a}$, L. Giommi $^{a, b}{ }^{,}$ C. Grandi ${ }^{a}$, L. Guiducci ${ }^{a, b}$, S. Lo Meo ${ }^{a, 41}$, L. Lunerti ${ }^{a, b}$, S. Marcellini ${ }^{a}$, G. Masetti ${ }^{a}$, F.L. Navarria ${ }^{a, b}$, A. Perrotta ${ }^{a}$, F. Primavera ${ }^{a, b}$, A.M. Rossi ${ }^{a, b}$, T. Rovelli ${ }^{a, b}$, G.P. Siroli ${ }^{a, b}$

INFN Sezione di Catania $^{a}$, Università di Catania ${ }^{b}$, Catania, Italy

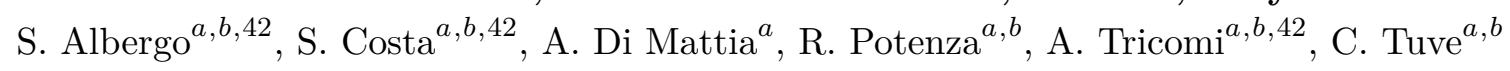
INFN Sezione di Firenze ${ }^{a}$, Università di Firenze ${ }^{b}$, Firenze, Italy

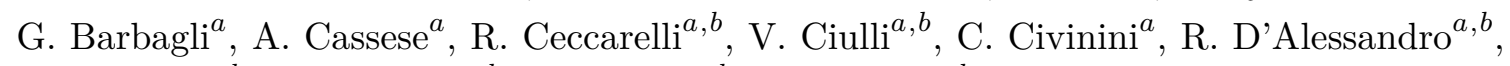
E. Focardi ${ }^{a, b}$, G. Latino ${ }^{a, b}$, P. Lenzi ${ }^{a, b}$, M. Lizzo ${ }^{a, b}$, M. Meschini ${ }^{a}$, S. Paoletti ${ }^{a}$, R. Seidita ${ }^{a, b}$, G. Sguazzoni ${ }^{a}$, L. Viliani $^{a}$

INFN Laboratori Nazionali di Frascati, Frascati, Italy

L. Benussi, S. Bianco, D. Piccolo

INFN Sezione di Genova ${ }^{a}$, Università di Genova ${ }^{b}$, Genova, Italy

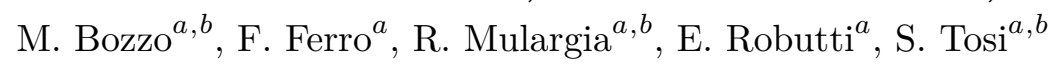

INFN Sezione di Milano-Bicocca ${ }^{a}$, Università di Milano-Bicocca ${ }^{b}$, Milano, Italy

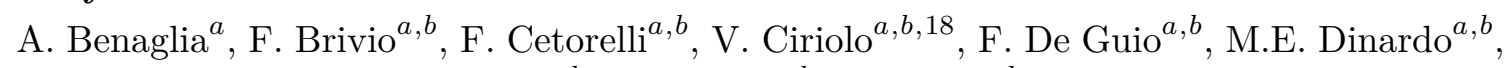
P. Dini ${ }^{a}$, S. Gennai ${ }^{a}$, A. Ghezzi ${ }^{a, b}$, P. Govoni $^{a}, b$, L. Guzzi $^{a}, b$, M. Malberti $^{a}$, S. Malvezzi $^{a}$, A. Massironi ${ }^{a}$, D. Menasce ${ }^{a}$, L. Moroni ${ }^{a}$, M. Paganoni ${ }^{a, b}$, D. Pedrini ${ }^{a}$, S. Ragazzi ${ }^{a, b}$, N. Redaelli ${ }^{a}$, T. Tabarelli de Fatis ${ }^{a, b}$, D. Valsecchi ${ }^{a, b, 18}$, D. Zuolo ${ }^{a, b}$

INFN Sezione di Napoli ${ }^{a}$, Università di Napoli 'Federico II' ${ }^{b}$, Napoli, Italy, Università della Basilicata ${ }^{c}$, Potenza, Italy, Università G. Marconi ${ }^{d}$, Roma, Italy

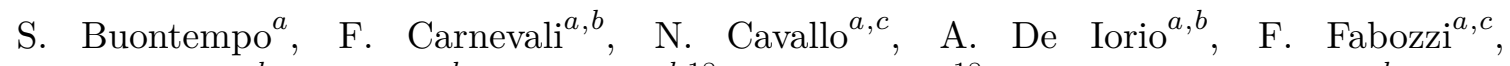

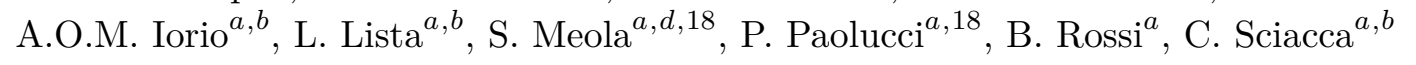

INFN Sezione di Padova ${ }^{a}$, Università di Padova ${ }^{b}$, Padova, Italy, Università di Trento ${ }^{c}$, Trento, Italy

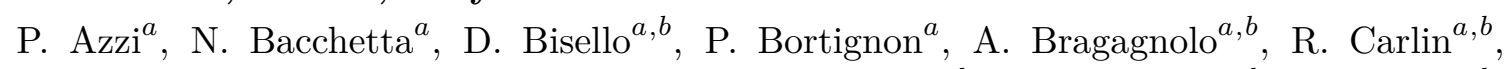

P. Checchia ${ }^{a}$, T. Dorigo ${ }^{a}$, U. Dosselli ${ }^{a}$, F. Gasparini ${ }^{a, b}$, U. Gasparini ${ }^{a, b}$, S.Y. Hoh $^{a, b}$,

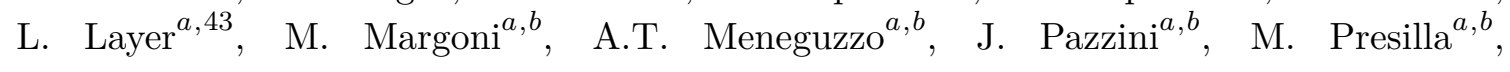




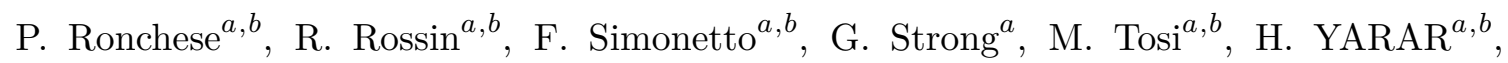
M. Zanetti ${ }^{a, b}$, P. Zotto ${ }^{a, b}$, A. Zucchetta $^{a, b}$, G. Zumerle ${ }^{a, b}$

INFN Sezione di Pavia ${ }^{a}$, Università di Pavia ${ }^{b}$

C. Aime ${ }^{a, b}$, A. Braghieri ${ }^{a}$, S. Calzaferri ${ }^{a, b}$, D. Fiorina ${ }^{a, b}$, P. Montagna ${ }^{a, b}$, S.P. Ratti ${ }^{a, b}$,

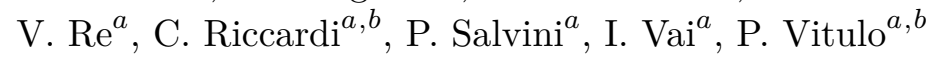

INFN Sezione di Perugia ${ }^{a}$, Università di Perugia ${ }^{b}$, Perugia, Italy

P. Asenov ${ }^{a, 44}$, G.M. Bilei ${ }^{a}$, D. Ciangottini ${ }^{a, b}$, L. Fanò ${ }^{a, b}$, P. Lariccia ${ }^{a, b}$, M. Magherini $^{b}$,

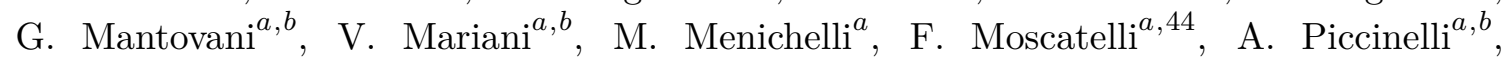

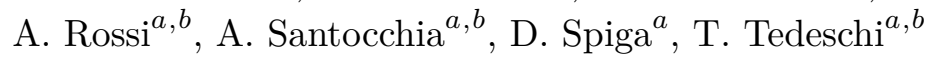

INFN Sezione di Pisa ${ }^{a}$, Università di Pisa ${ }^{b}$, Scuola Normale Superiore di Pisa ${ }^{c}$, Pisa Italy, Università di Siena ${ }^{d}$, Siena, Italy

P. Azzurri ${ }^{a}$, G. Bagliesi ${ }^{a}$, V. Bertacchi ${ }^{a, c}$, L. Bianchini ${ }^{a}$, T. Boccali ${ }^{a}$, E. Bossini ${ }^{a, b}$, R. Castaldi $^{a}$, M.A. Ciocci ${ }^{a, b}$, V. D'Amante ${ }^{a, d}$, R. Dell'Orso ${ }^{a}$, M.R. Di Domenico ${ }^{a, d}$, S. Donato ${ }^{a}$, A. Giassi ${ }^{a}$, F. Ligabue ${ }^{a, c}$, E. Manca ${ }^{a, c}$, G. Mandorli ${ }^{a, c}$, A. Messineo $^{a, b}$,

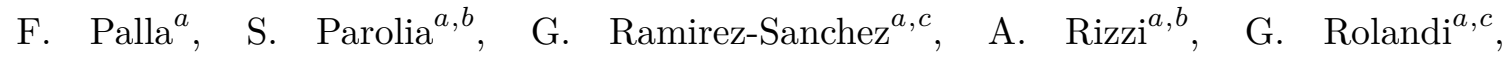
S. Roy Chowdhury ${ }^{a, c}$, A. Scribano ${ }^{a}$, N. Shafiei ${ }^{a, b}$, P. Spagnolo $^{a}$, R. Tenchini ${ }^{a}$, G. Tonelli $^{a, b}{ }$ N. Turini ${ }^{a, d}$, A. Venturi ${ }^{a}$, P.G. Verdini ${ }^{a}$

INFN Sezione di Roma ${ }^{a}$, Sapienza Università di Roma ${ }^{b}$, Rome, Italy M. Campana ${ }^{a, b}$, F. Cavallari ${ }^{a}$, D. Del Re ${ }^{a, b}$, E. Di Marco ${ }^{a}$, M. Diemoz $^{a}$, E. Longo ${ }^{a, b}$, P. Meridiani ${ }^{a}$, G. Organtini ${ }^{a, b}$, F. Pandolfi ${ }^{a}$, R. Paramatti ${ }^{a, b}$, C. Quaranta ${ }^{a, b}$,

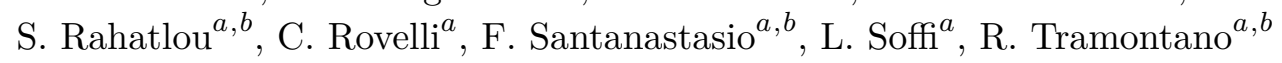

INFN Sezione di Torino ${ }^{a}$, Università di Torino ${ }^{b}$, Torino, Italy, Università del Piemonte Orientale ${ }^{c}$, Novara, Italy

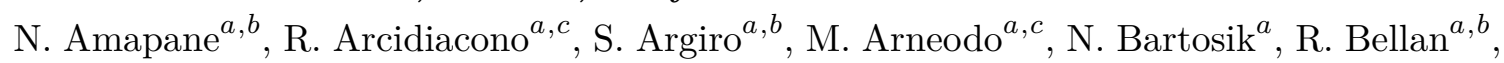

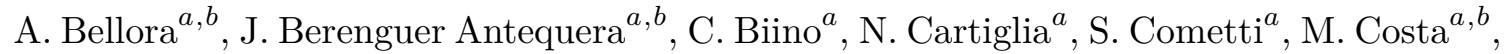
R. Covarelli ${ }^{a, b}$, N. Demaria ${ }^{a}$, B. Kiani ${ }^{a, b}$, F. Legger ${ }^{a}$, C. Mariotti ${ }^{a}$, S. Maselli ${ }^{a}$, E. Migliore $^{a, b}$, E. Monteil ${ }^{a, b}$, M. Monteno ${ }^{a}$, M.M. Obertino ${ }^{a, b}$, G. Ortona $^{a}$, L. Pacher ${ }^{a, b}$, N. Pastrone $^{a}$, M. Pelliccioni ${ }^{a}$, G.L. Pinna Angioni ${ }^{a, b}$, M. Ruspa ${ }^{a, c}$, K. Shchelina ${ }^{a, b}$,

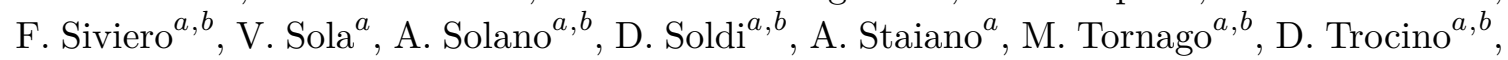
A. Vagnerini

INFN Sezione di Trieste ${ }^{a}$, Università di Trieste ${ }^{b}$, Trieste, Italy

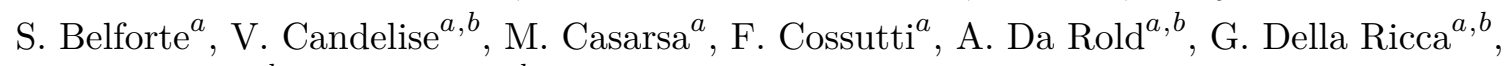
G. Sorrentino $^{a, b}$, F. Vazzoler ${ }^{a, b}$

\section{Kyungpook National University, Daegu, Korea}

S. Dogra, C. Huh, B. Kim, D.H. Kim, G.N. Kim, J. Kim, J. Lee, S.W. Lee, C.S. Moon, Y.D. Oh, S.I. Pak, B.C. Radburn-Smith, S. Sekmen, Y.C. Yang

Chonnam National University, Institute for Universe and Elementary Particles, Kwangju, Korea

H. Kim, D.H. Moon 
Hanyang University, Seoul, Korea

B. Francois, T.J. Kim, J. Park

Korea University, Seoul, Korea

S. Cho, S. Choi, Y. Go, B. Hong, K. Lee, K.S. Lee, J. Lim, J. Park, S.K. Park, J. Yoo

Kyung Hee University, Department of Physics, Seoul, Republic of Korea

J. Goh, A. Gurtu

Sejong University, Seoul, Korea

H.S. Kim, Y. Kim

Seoul National University, Seoul, Korea

J. Almond, J.H. Bhyun, J. Choi, S. Jeon, J. Kim, J.S. Kim, S. Ko, H. Kwon, H. Lee, S. Lee, B.H. Oh, M. Oh, S.B. Oh, H. Seo, U.K. Yang, I. Yoon

University of Seoul, Seoul, Korea

W. Jang, D. Jeon, D.Y. Kang, Y. Kang, J.H. Kim, S. Kim, B. Ko, J.S.H. Lee, Y. Lee, I.C. Park, Y. Roh, M.S. Ryu, D. Song, I.J. Watson, S. Yang

Yonsei University, Department of Physics, Seoul, Korea

S. Ha, H.D. Yoo

Sungkyunkwan University, Suwon, Korea

M. Choi, Y. Jeong, H. Lee, Y. Lee, I. Yu

College of Engineering and Technology, American University of the Middle East (AUM), Egaila, Kuwait

T. Beyrouthy, Y. Maghrbi

Riga Technical University

T. Torims, V. Veckalns ${ }^{45}$

Vilnius University, Vilnius, Lithuania

M. Ambrozas, A. Carvalho Antunes De Oliveira, A. Juodagalvis, A. Rinkevicius, G. Tamulaitis

National Centre for Particle Physics, Universiti Malaya, Kuala Lumpur, Malaysia

N. Bin Norjoharuddeen, W.A.T. Wan Abdullah, M.N. Yusli, Z. Zolkapli

Universidad de Sonora (UNISON), Hermosillo, Mexico

J.F. Benitez, A. Castaneda Hernandez, M. León Coello, J.A. Murillo Quijada, A. Sehrawat, L. Valencia Palomo

Centro de Investigacion y de Estudios Avanzados del IPN, Mexico City, Mexico G. Ayala, H. Castilla-Valdez, E. De La Cruz-Burelo, I. Heredia-De La Cruz ${ }^{46}$, R. LopezFernandez, C.A. Mondragon Herrera, D.A. Perez Navarro, A. Sanchez-Hernandez

Universidad Iberoamericana, Mexico City, Mexico

S. Carrillo Moreno, C. Oropeza Barrera, M. Ramirez-Garcia, F. Vazquez Valencia 
Benemerita Universidad Autonoma de Puebla, Puebla, Mexico

I. Pedraza, H.A. Salazar Ibarguen, C. Uribe Estrada

University of Montenegro, Podgorica, Montenegro

J. Mijuskovic ${ }^{47}$, N. Raicevic

University of Auckland, Auckland, New Zealand

D. Krofcheck

University of Canterbury, Christchurch, New Zealand

S. Bheesette, P.H. Butler

National Centre for Physics, Quaid-I-Azam University, Islamabad, Pakistan

A. Ahmad, M.I. Asghar, A. Awais, M.I.M. Awan, H.R. Hoorani, W.A. Khan, M.A. Shah, M. Shoaib, M. Waqas

AGH University of Science and Technology Faculty of Computer Science, Electronics and Telecommunications, Krakow, Poland

V. Avati, L. Grzanka, M. Malawski

National Centre for Nuclear Research, Swierk, Poland

H. Bialkowska, M. Bluj, B. Boimska, M. Górski, M. Kazana, M. Szleper, P. Zalewski

Institute of Experimental Physics, Faculty of Physics, University of Warsaw, Warsaw, Poland

K. Bunkowski, K. Doroba, A. Kalinowski, M. Konecki, J. Krolikowski, M. Walczak

Laboratório de Instrumentação e Física Experimental de Partículas, Lisboa, Portugal

M. Araujo, P. Bargassa, D. Bastos, A. Boletti, P. Faccioli, M. Gallinaro, J. Hollar, N. Leonardo, T. Niknejad, M. Pisano, J. Seixas, O. Toldaiev, J. Varela

Joint Institute for Nuclear Research, Dubna, Russia

S. Afanasiev, D. Budkouski, I. Golutvin, I. Gorbunov, V. Karjavine, V. Korenkov, A. Lanev, A. Malakhov, V. Matveev ${ }^{48,49}$, V. Palichik, V. Perelygin, M. Savina, D. Seitova, V. Shalaev, S. Shmatov, S. Shulha, V. Smirnov, O. Teryaev, N. Voytishin, B.S. Yuldashev ${ }^{50}$, A. Zarubin, I. Zhizhin

Petersburg Nuclear Physics Institute, Gatchina (St. Petersburg), Russia

G. Gavrilov, V. Golovtcov, Y. Ivanov, V. Kim ${ }^{51}$, E. Kuznetsova ${ }^{52}$, V. Murzin, V. Oreshkin, I. Smirnov, D. Sosnov, V. Sulimov, L. Uvarov, S. Volkov, A. Vorobyev

Institute for Nuclear Research, Moscow, Russia

Yu. Andreev, A. Dermenev, S. Gninenko, N. Golubev, A. Karneyeu, D. Kirpichnikov, M. Kirsanov, N. Krasnikov, A. Pashenkov, G. Pivovarov, D. Tlisov ${ }^{\dagger}$, A. Toropin

Institute for Theoretical and Experimental Physics named by A.I. Alikhanov of NRC 'Kurchatov Institute', Moscow, Russia

V. Epshteyn, V. Gavrilov, N. Lychkovskaya, A. Nikitenko ${ }^{53}$, V. Popov, A. Spiridonov, A. Stepennov, M. Toms, E. Vlasov, A. Zhokin 
Moscow Institute of Physics and Technology, Moscow, Russia

T. Aushev

National Research Nuclear University 'Moscow Engineering Physics Institute' (MEPhI), Moscow, Russia

R. Chistov ${ }^{54}$, M. Danilov ${ }^{55}$, A. Oskin, P. Parygin, S. Polikarpov ${ }^{54}$

P.N. Lebedev Physical Institute, Moscow, Russia

V. Andreev, M. Azarkin, I. Dremin, M. Kirakosyan, A. Terkulov

Skobeltsyn Institute of Nuclear Physics, Lomonosov Moscow State University, Moscow, Russia

A. Belyaev, E. Boos, V. Bunichev, M. Dubinin ${ }^{56}$, L. Dudko, V. Klyukhin, O. Kodolova,

N. Korneeva, I. Lokhtin, S. Obraztsov, M. Perfilov, V. Savrin, P. Volkov

Novosibirsk State University (NSU), Novosibirsk, Russia

V. Blinov ${ }^{57}$, T. Dimova ${ }^{57}$, L. Kardapoltsev ${ }^{57}$, A. Kozyrev ${ }^{57}$, I. Ovtin ${ }^{57}$, Y. Skovpen ${ }^{57}$

Institute for High Energy Physics of National Research Centre 'Kurchatov Institute', Protvino, Russia

I. Azhgirey, I. Bayshev, D. Elumakhov, V. Kachanov, D. Konstantinov, P. Mandrik,

V. Petrov, R. Ryutin, S. Slabospitskii, A. Sobol, S. Troshin, N. Tyurin, A. Uzunian,

A. Volkov

National Research Tomsk Polytechnic University, Tomsk, Russia

A. Babaev, V. Okhotnikov

Tomsk State University, Tomsk, Russia

V. Borshch, V. Ivanchenko, E. Tcherniaev

University of Belgrade: Faculty of Physics and VINCA Institute of Nuclear Sciences, Belgrade, Serbia

P. Adzic ${ }^{58}$, M. Dordevic, P. Milenovic, J. Milosevic

Centro de Investigaciones Energéticas Medioambientales y Tecnológicas (CIEMAT), Madrid, Spain

M. Aguilar-Benitez, J. Alcaraz Maestre, A. Álvarez Fernández, I. Bachiller, M. Barrio Luna, Cristina F. Bedoya, C.A. Carrillo Montoya, M. Cepeda, M. Cerrada, N. Colino, B. De La Cruz, A. Delgado Peris, J.P. Fernández Ramos, J. Flix, M.C. Fouz, O. Gonzalez Lopez, S. Goy Lopez, J.M. Hernandez, M.I. Josa, J. León Holgado, D. Moran, Á. Navarro Tobar, C. Perez Dengra, A. Pérez-Calero Yzquierdo, J. Puerta Pelayo, I. Redondo, L. Romero, S. Sánchez Navas, L. Urda Gómez, C. Willmott

Universidad Autónoma de Madrid, Madrid, Spain

J.F. de Trocóniz, R. Reyes-Almanza 
Universidad de Oviedo, Instituto Universitario de Ciencias y Tecnologías Espaciales de Asturias (ICTEA), Oviedo, Spain

B. Alvarez Gonzalez, J. Cuevas, C. Erice, J. Fernandez Menendez, S. Folgueras, I. Gonzalez Caballero, J.R. González Fernández, E. Palencia Cortezon, C. Ramón Álvarez, J. Ripoll Sau, V. Rodríguez Bouza, A. Trapote, N. Trevisani

Instituto de Física de Cantabria (IFCA), CSIC-Universidad de Cantabria, Santander, Spain

J.A. Brochero Cifuentes, I.J. Cabrillo, A. Calderon, J. Duarte Campderros, M. Fernandez, C. Fernandez Madrazo, P.J. Fernández Manteca, A. García Alonso, G. Gomez, C. Martinez Rivero, P. Martinez Ruiz del Arbol, F. Matorras, P. Matorras Cuevas,

J. Piedra Gomez, C. Prieels, T. Rodrigo, A. Ruiz-Jimeno, L. Scodellaro, I. Vila, J.M. Vizan Garcia

\section{University of Colombo, Colombo, Sri Lanka}

MK Jayananda, B. Kailasapathy ${ }^{59}$, D.U.J. Sonnadara, DDC Wickramarathna

University of Ruhuna, Department of Physics, Matara, Sri Lanka

W.G.D. Dharmaratna, K. Liyanage, N. Perera, N. Wickramage

CERN, European Organization for Nuclear Research, Geneva, Switzerland

T.K. Aarrestad, D. Abbaneo, J. Alimena, E. Auffray, G. Auzinger, J. Baechler, P. Baillon ${ }^{\dagger}$,

D. Barney, J. Bendavid, M. Bianco, A. Bocci, T. Camporesi, M. Capeans Garrido,

G. Cerminara, S.S. Chhibra, M. Cipriani, L. Cristella, D. d'Enterria, A. Dabrowski,

N. Daci, A. David, A. De Roeck, M.M. Defranchis, M. Deile, M. Dobson, M. Dünser,

N. Dupont, A. Elliott-Peisert, N. Emriskova, F. Fallavollita ${ }^{60}$, D. Fasanella, A. Florent,

G. Franzoni, W. Funk, S. Giani, D. Gigi, K. Gill, F. Glege, L. Gouskos, M. Haranko,

J. Hegeman, Y. Iiyama, V. Innocente, T. James, P. Janot, J. Kaspar, J. Kieseler, M. Komm,

N. Kratochwil, C. Lange, S. Laurila, P. Lecoq, K. Long, C. Lourenço, L. Malgeri,

S. Mallios, M. Mannelli, A.C. Marini, F. Meijers, S. Mersi, E. Meschi, F. Moortgat,

M. Mulders, S. Orfanelli, L. Orsini, F. Pantaleo, L. Pape, E. Perez, M. Peruzzi, A. Petrilli,

G. Petrucciani, A. Pfeiffer, M. Pierini, D. Piparo, M. Pitt, H. Qu, T. Quast, D. Rabady,

A. Racz, G. Reales Gutiérrez, M. Rieger, M. Rovere, H. Sakulin, J. Salfeld-Nebgen, S. Scarfi, C. Schäfer, C. Schwick, M. Selvaggi, A. Sharma, P. Silva, W. Snoeys, P. Sphicas ${ }^{61}$, S. Summers, K. Tatar, V.R. Tavolaro, D. Treille, A. Tsirou, G.P. Van Onsem, M. Verzetti, J. Wanczyk ${ }^{62}$, K.A. Wozniak, W.D. Zeuner

Paul Scherrer Institut, Villigen, Switzerland

L. Caminada ${ }^{63}$, A. Ebrahimi, W. Erdmann, R. Horisberger, Q. Ingram, H.C. Kaestli, D. Kotlinski, U. Langenegger, M. Missiroli, T. Rohe

ETH Zurich - Institute for Particle Physics and Astrophysics (IPA), Zurich, Switzerland

K. Androsov ${ }^{62}$, M. Backhaus, P. Berger, A. Calandri, N. Chernyavskaya, A. De Cosa, G. Dissertori, M. Dittmar, M. Donegà, C. Dorfer, F. Eble, K. Gedia, F. Glessgen, T.A. Gómez Espinosa, C. Grab, D. Hits, W. Lustermann, A.-M. Lyon, R.A. Manzoni, 
C. Martin Perez, M.T. Meinhard, F. Nessi-Tedaldi, J. Niedziela, F. Pauss, V. Perovic, S. Pigazzini, M.G. Ratti, M. Reichmann, C. Reissel, T. Reitenspiess, B. Ristic, D. Ruini, D.A. Sanz Becerra, M. Schönenberger, V. Stampf, J. Steggemann ${ }^{62}$, R. Wallny, D.H. Zhu

Universität Zürich, Zurich, Switzerland

C. Amsler ${ }^{64}$, P. Bärtschi, C. Botta, D. Brzhechko, M.F. Canelli, K. Cormier, A. De Wit,

R. Del Burgo, J.K. Heikkilä, M. Huwiler, W. Jin, A. Jofrehei, B. Kilminster, S. Leontsinis, S.P. Liechti, A. Macchiolo, P. Meiring, V.M. Mikuni, U. Molinatti, I. Neutelings, A. Reimers, P. Robmann, S. Sanchez Cruz, K. Schweiger, Y. Takahashi

National Central University, Chung-Li, Taiwan

C. Adloff ${ }^{65}$, C.M. Kuo, W. Lin, A. Roy, T. Sarkar ${ }^{35}$, S.S. Yu

National Taiwan University (NTU), Taipei, Taiwan

L. Ceard, Y. Chao, K.F. Chen, P.H. Chen, W.-S. Hou, Y.y. Li, R.-S. Lu, E. Paganis, A. Psallidas, A. Steen, H.y. Wu, E. Yazgan, P.r. Yu

Chulalongkorn University, Faculty of Science, Department of Physics, Bangkok, Thailand

B. Asavapibhop, C. Asawatangtrakuldee, N. Srimanobhas

Çukurova University, Physics Department, Science and Art Faculty, Adana, Turkey

F. Boran, S. Damarseckin ${ }^{66}$, Z.S. Demiroglu, F. Dolek, I. Dumanoglu ${ }^{67}$, E. Eskut, Y. Guler,

E. Gurpinar Guler ${ }^{68}$, I. Hos $^{69}$, C. Isik, O. Kara, A. Kayis Topaksu, U. Kiminsu, G. Onengut,

K. Ozdemir ${ }^{70}$, A. Polatoz, A.E. Simsek, B. Tali ${ }^{71}$, U.G. Tok, S. Turkcapar, I.S. Zorbakir,

C. Zorbilmez

Middle East Technical University, Physics Department, Ankara, Turkey

B. Isildak ${ }^{72}$, G. Karapinar ${ }^{73}$, K. Ocalan ${ }^{74}$, M. Yalvac ${ }^{75}$

Bogazici University, Istanbul, Turkey

B. Akgun, I.O. Atakisi, E. Gülmez, M. Kaya ${ }^{76}$, O. Kaya ${ }^{77}$, Ö. Özçelik, S. Tekten ${ }^{78}$, E.A. Yetkin ${ }^{79}$

Istanbul Technical University, Istanbul, Turkey

A. Cakir, K. Cankocak ${ }^{67}$, Y. Komurcu, S. Sen ${ }^{80}$

Istanbul University, Istanbul, Turkey

S. Cerci ${ }^{71}$, B. Kaynak, S. Ozkorucuklu, D. Sunar Cerci ${ }^{71}$

Institute for Scintillation Materials of National Academy of Science of Ukraine, Kharkov, Ukraine

B. Grynyov

National Scientific Center, Kharkov Institute of Physics and Technology, Kharkov, Ukraine

L. Levchuk 


\section{University of Bristol, Bristol, United Kingdom}

D. Anthony, E. Bhal, S. Bologna, J.J. Brooke, A. Bundock, E. Clement, D. Cussans, H. Flacher, J. Goldstein, G.P. Heath, H.F. Heath, M.l. Holmberg ${ }^{81}$, L. Kreczko, B. Krikler, S. Paramesvaran, S. Seif El Nasr-Storey, V.J. Smith, N. Stylianou ${ }^{82}$, K. Walkingshaw Pass, R. White

\section{Rutherford Appleton Laboratory, Didcot, United Kingdom}

K.W. Bell, A. Belyaev ${ }^{83}$, C. Brew, R.M. Brown, D.J.A. Cockerill, C. Cooke, K.V. Ellis, K. Harder, S. Harper, J. Linacre, K. Manolopoulos, D.M. Newbold, E. Olaiya, D. Petyt, T. Reis, T. Schuh, C.H. Shepherd-Themistocleous, I.R. Tomalin, T. Williams

\section{Imperial College, London, United Kingdom}

R. Bainbridge, P. Bloch, S. Bonomally, J. Borg, S. Breeze, O. Buchmuller, V. Cepaitis, G.S. Chahal ${ }^{84}$, D. Colling, P. Dauncey, G. Davies, M. Della Negra, S. Fayer, G. Fedi, G. Hall, M.H. Hassanshahi, G. Iles, J. Langford, L. Lyons, A.-M. Magnan, S. Malik, A. Martelli, D.G. Monk, J. Nash ${ }^{85}$, M. Pesaresi, D.M. Raymond, A. Richards, A. Rose, E. Scott, C. Seez, A. Shtipliyski, A. Tapper, K. Uchida, T. Virdee ${ }^{18}$, M. Vojinovic, N. Wardle, S.N. Webb, D. Winterbottom, A.G. Zecchinelli

\section{Brunel University, Uxbridge, United Kingdom}

K. Coldham, J.E. Cole, A. Khan, P. Kyberd, I.D. Reid, L. Teodorescu, S. Zahid

Baylor University, Waco, U.S.A.

S. Abdullin, A. Brinkerhoff, B. Caraway, J. Dittmann, K. Hatakeyama, A.R. Kanuganti, B. McMaster, N. Pastika, M. Saunders, S. Sawant, C. Sutantawibul, J. Wilson

Catholic University of America, Washington, DC, U.S.A.

R. Bartek, A. Dominguez, R. Uniyal, A.M. Vargas Hernandez

The University of Alabama, Tuscaloosa, U.S.A.

A. Buccilli, S.I. Cooper, D. Di Croce, S.V. Gleyzer, C. Henderson, C.U. Perez, P. Rumerio ${ }^{86}$, C. West

Boston University, Boston, U.S.A.

A. Akpinar, A. Albert, D. Arcaro, C. Cosby, Z. Demiragli, E. Fontanesi, D. Gastler, J. Rohlf, K. Salyer, D. Sperka, D. Spitzbart, I. Suarez, A. Tsatsos, S. Yuan, D. Zou

\section{Brown University, Providence, U.S.A.}

G. Benelli, B. Burkle, X. Coubez ${ }^{19}$, D. Cutts, M. Hadley, U. Heintz, J.M. Hogan ${ }^{87}$, G. Landsberg, K.T. Lau, M. Lukasik, J. Luo, M. Narain, S. Sagir ${ }^{88}$, E. Usai, W.Y. Wong, X. Yan, D. Yu, W. Zhang

University of California, Davis, Davis, U.S.A.

J. Bonilla, C. Brainerd, R. Breedon, M. Calderon De La Barca Sanchez, M. Chertok, J. Conway, P.T. Cox, R. Erbacher, G. Haza, F. Jensen, O. Kukral, R. Lander, M. Mulhearn, D. Pellett, B. Regnery, D. Taylor, Y. Yao, F. Zhang 
University of California, Los Angeles, U.S.A.

M. Bachtis, R. Cousins, A. Datta, D. Hamilton, J. Hauser, M. Ignatenko, M.A. Iqbal, T. Lam, W.A. Nash, S. Regnard, D. Saltzberg, B. Stone, V. Valuev

University of California, Riverside, Riverside, U.S.A.

K. Burt, Y. Chen, R. Clare, J.W. Gary, M. Gordon, G. Hanson, G. Karapostoli, O.R. Long, N. Manganelli, M. Olmedo Negrete, W. Si, S. Wimpenny, Y. Zhang

University of California, San Diego, La Jolla, U.S.A.

J.G. Branson, P. Chang, S. Cittolin, S. Cooperstein, N. Deelen, D. Diaz, J. Duarte, R. Gerosa, L. Giannini, D. Gilbert, J. Guiang, R. Kansal, V. Krutelyov, R. Lee, J. Letts, M. Masciovecchio, S. May, M. Pieri, B.V. Sathia Narayanan, V. Sharma, M. Tadel, A. Vartak, F. Würthwein, Y. Xiang, A. Yagil

University of California, Santa Barbara - Department of Physics, Santa Barbara, U.S.A.

N. Amin, C. Campagnari, M. Citron, A. Dorsett, V. Dutta, J. Incandela, M. Kilpatrick, J. Kim, B. Marsh, H. Mei, M. Oshiro, M. Quinnan, J. Richman, U. Sarica, F. Setti, J. Sheplock, D. Stuart, S. Wang

California Institute of Technology, Pasadena, U.S.A.

A. Bornheim, O. Cerri, I. Dutta, J.M. Lawhorn, N. Lu, J. Mao, H.B. Newman, T.Q. Nguyen, M. Spiropulu, J.R. Vlimant, C. Wang, S. Xie, Z. Zhang, R.Y. Zhu

Carnegie Mellon University, Pittsburgh, U.S.A.

J. Alison, S. An, M.B. Andrews, P. Bryant, T. Ferguson, A. Harilal, C. Liu, T. Mudholkar, M. Paulini, A. Sanchez, W. Terrill

University of Colorado Boulder, Boulder, U.S.A.

J.P. Cumalat, W.T. Ford, A. Hassani, E. MacDonald, R. Patel, A. Perloff, C. Savard, K. Stenson, K.A. Ulmer, S.R. Wagner

Cornell University, Ithaca, U.S.A.

J. Alexander, S. Bright-thonney, Y. Cheng, D.J. Cranshaw, S. Hogan, J. Monroy, J.R. Patterson, D. Quach, J. Reichert, M. Reid, A. Ryd, W. Sun, J. Thom, P. Wittich, R. Zou

Fermi National Accelerator Laboratory, Batavia, U.S.A.

M. Albrow, M. Alyari, G. Apollinari, A. Apresyan, A. Apyan, S. Banerjee, L.A.T. Bauerdick, D. Berry, J. Berryhill, P.C. Bhat, K. Burkett, J.N. Butler, A. Canepa, G.B. Cerati, H.W.K. Cheung, F. Chlebana, M. Cremonesi, K.F. Di Petrillo, V.D. Elvira, Y. Feng, J. Freeman, Z. Gecse, L. Gray, D. Green, S. Grünendahl, O. Gutsche, R.M. Harris, R. Heller, T.C. Herwig, J. Hirschauer, B. Jayatilaka, S. Jindariani, M. Johnson, U. Joshi, T. Klijnsma, B. Klima, K.H.M. Kwok, S. Lammel, D. Lincoln, R. Lipton, T. Liu, C. Madrid, K. Maeshima, C. Mantilla, D. Mason, P. McBride, P. Merkel, S. Mrenna, S. Nahn, J. Ngadiuba, V. O'Dell, V. Papadimitriou, K. Pedro, C. Pena ${ }^{56}$, O. Prokofyev, F. Ravera, A. Reinsvold Hall, L. Ristori, B. Schneider, E. Sexton-Kennedy, N. Smith, A. Soha, W.J. Spalding, L. Spiegel, S. Stoynev, J. Strait, L. Taylor, S. Tkaczyk, N.V. Tran, L. Uplegger, E.W. Vaandering, H.A. Weber 
University of Florida, Gainesville, U.S.A.

D. Acosta, P. Avery, D. Bourilkov, L. Cadamuro, V. Cherepanov, F. Errico, R.D. Field,

D. Guerrero, B.M. Joshi, M. Kim, E. Koenig, J. Konigsberg, A. Korytov, K.H. Lo,

K. Matchev, N. Menendez, G. Mitselmakher, A. Muthirakalayil Madhu, N. Rawal,

D. Rosenzweig, S. Rosenzweig, K. Shi, J. Sturdy, J. Wang, E. Yigitbasi, X. Zuo

Florida State University, Tallahassee, U.S.A.

T. Adams, A. Askew, R. Habibullah, V. Hagopian, K.F. Johnson, R. Khurana, T. Kolberg,

G. Martinez, H. Prosper, C. Schiber, O. Viazlo, R. Yohay, J. Zhang

Florida Institute of Technology, Melbourne, U.S.A.

M.M. Baarmand, S. Butalla, T. Elkafrawy ${ }^{13}$, M. Hohlmann, R. Kumar Verma, D. Noonan, M. Rahmani, F. Yumiceva

University of Illinois at Chicago (UIC), Chicago, U.S.A.

M.R. Adams, H. Becerril Gonzalez, R. Cavanaugh, X. Chen, S. Dittmer, O. Evdokimov, C.E. Gerber, D.A. Hangal, D.J. Hofman, A.H. Merrit, C. Mills, G. Oh, T. Roy, S. Rudrabhatla, M.B. Tonjes, N. Varelas, J. Viinikainen, X. Wang, Z. Wu, Z. Ye

The University of Iowa, Iowa City, U.S.A.

M. Alhusseini, K. Dilsiz ${ }^{89}$, R.P. Gandrajula, O.K. Köseyan, J.-P. Merlo, A. Mestvirishvili ${ }^{90}$, J. Nachtman, H. Ogul ${ }^{91}$, Y. Onel, A. Penzo, C. Snyder, E. Tiras ${ }^{92}$

Johns Hopkins University, Baltimore, U.S.A.

O. Amram, B. Blumenfeld, L. Corcodilos, J. Davis, M. Eminizer, A.V. Gritsan, S. Kyriacou, P. Maksimovic, J. Roskes, M. Swartz, T.Á. Vámi

The University of Kansas, Lawrence, U.S.A.

A. Abreu, J. Anguiano, C. Baldenegro Barrera, P. Baringer, A. Bean, A. Bylinkin, Z. Flowers, T. Isidori, S. Khalil, J. King, G. Krintiras, A. Kropivnitskaya, M. Lazarovits, C. Lindsey, J. Marquez, N. Minafra, M. Murray, M. Nickel, C. Rogan, C. Royon, R. Salvatico, S. Sanders, E. Schmitz, C. Smith, J.D. Tapia Takaki, Q. Wang, Z. Warner, J. Williams, G. Wilson

Kansas State University, Manhattan, U.S.A.

S. Duric, A. Ivanov, K. Kaadze, D. Kim, Y. Maravin, T. Mitchell, A. Modak, K. Nam

Lawrence Livermore National Laboratory, Livermore, U.S.A.

F. Rebassoo, D. Wright

University of Maryland, College Park, U.S.A.

E. Adams, A. Baden, O. Baron, A. Belloni, S.C. Eno, N.J. Hadley, S. Jabeen, R.G. Kellogg, T. Koeth, A.C. Mignerey, S. Nabili, C. Palmer, M. Seidel, A. Skuja, L. Wang, K. Wong

Massachusetts Institute of Technology, Cambridge, U.S.A.

D. Abercrombie, G. Andreassi, R. Bi, S. Brandt, W. Busza, I.A. Cali, Y. Chen, M. D'Alfonso, J. Eysermans, C. Freer, G. Gomez Ceballos, M. Goncharov, P. Harris, M. Hu, M. Klute, D. Kovalskyi, J. Krupa, Y.-J. Lee, B. Maier, C. Mironov, C. Paus, D. Rankin, C. Roland, G. Roland, Z. Shi, G.S.F. Stephans, J. Wang, Z. Wang, B. Wyslouch 
University of Minnesota, Minneapolis, U.S.A.

R.M. Chatterjee, A. Evans, P. Hansen, J. Hiltbrand, Sh. Jain, M. Krohn, Y. Kubota, J. Mans, M. Revering, R. Rusack, R. Saradhy, N. Schroeder, N. Strobbe, M.A. Wadud

University of Nebraska-Lincoln, Lincoln, U.S.A.

K. Bloom, M. Bryson, S. Chauhan, D.R. Claes, C. Fangmeier, L. Finco, F. Golf, C. Joo, I. Kravchenko, M. Musich, I. Reed, J.E. Siado, G.R. Snow ${ }^{\dagger}$, W. Tabb, F. Yan

State University of New York at Buffalo, Buffalo, U.S.A.

G. Agarwal, H. Bandyopadhyay, L. Hay, I. Iashvili, A. Kharchilava, C. McLean, D. Nguyen, J. Pekkanen, S. Rappoccio, A. Williams

Northeastern University, Boston, U.S.A.

G. Alverson, E. Barberis, Y. Haddad, A. Hortiangtham, J. Li, G. Madigan, B. Marzocchi, D.M. Morse, V. Nguyen, T. Orimoto, A. Parker, L. Skinnari, A. Tishelman-Charny, T. Wamorkar, B. Wang, A. Wisecarver, D. Wood

Northwestern University, Evanston, U.S.A.

S. Bhattacharya, J. Bueghly, Z. Chen, A. Gilbert, T. Gunter, K.A. Hahn, Y. Liu, N. Odell, M.H. Schmitt, M. Velasco

University of Notre Dame, Notre Dame, U.S.A.

R. Band, R. Bucci, A. Das, N. Dev, R. Goldouzian, M. Hildreth, K. Hurtado Anampa, C. Jessop, K. Lannon, J. Lawrence, N. Loukas, D. Lutton, N. Marinelli, I. Mcalister, T. McCauley, C. Mcgrady, F. Meng, K. Mohrman, Y. Musienko ${ }^{48}$, R. Ruchti, P. Siddireddy, A. Townsend, M. Wayne, A. Wightman, M. Wolf, M. Zarucki, L. Zygala

The Ohio State University, Columbus, U.S.A.

B. Bylsma, B. Cardwell, L.S. Durkin, B. Francis, C. Hill, M. Nunez Ornelas, K. Wei, B.L. Winer, B.R. Yates

Princeton University, Princeton, U.S.A.

F.M. Addesa, B. Bonham, P. Das, G. Dezoort, P. Elmer, A. Frankenthal, B. Greenberg, N. Haubrich, S. Higginbotham, A. Kalogeropoulos, G. Kopp, S. Kwan, D. Lange, M.T. Lucchini, D. Marlow, K. Mei, I. Ojalvo, J. Olsen, D. Stickland, C. Tully

University of Puerto Rico, Mayaguez, U.S.A.

S. Malik, S. Norberg

Purdue University, West Lafayette, U.S.A.

A.S. Bakshi, V.E. Barnes, R. Chawla, S. Das, L. Gutay, M. Jones, A.W. Jung, S. Karmarkar, M. Liu, G. Negro, N. Neumeister, G. Paspalaki, C.C. Peng, S. Piperov, A. Purohit, J.F. Schulte, M. Stojanovic ${ }^{15}$, J. Thieman, F. Wang, R. Xiao, W. Xie

Purdue University Northwest, Hammond, U.S.A.

J. Dolen, N. Parashar 
Rice University, Houston, U.S.A.

A. Baty, M. Decaro, S. Dildick, K.M. Ecklund, S. Freed, P. Gardner, F.J.M. Geurts, A. Kumar, W. Li, B.P. Padley, R. Redjimi, W. Shi, A.G. Stahl Leiton, S. Yang, L. Zhang, Y. Zhang

University of Rochester, Rochester, U.S.A.

A. Bodek, P. de Barbaro, R. Demina, J.L. Dulemba, C. Fallon, T. Ferbel, M. Galanti, A. Garcia-Bellido, O. Hindrichs, A. Khukhunaishvili, E. Ranken, R. Taus

Rutgers, The State University of New Jersey, Piscataway, U.S.A.

B. Chiarito, J.P. Chou, A. Gandrakota, Y. Gershtein, E. Halkiadakis, A. Hart, M. Heindl, O. Karacheban ${ }^{22}$, I. Laflotte, A. Lath, R. Montalvo, K. Nash, M. Osherson, S. Salur, S. Schnetzer, S. Somalwar, R. Stone, S.A. Thayil, S. Thomas, H. Wang

University of Tennessee, Knoxville, U.S.A.

H. Acharya, A.G. Delannoy, S. Fiorendi, S. Spanier

Texas A\&M University, College Station, U.S.A.

O. Bouhali ${ }^{93}$, M. Dalchenko, A. Delgado, R. Eusebi, J. Gilmore, T. Huang, T. Kamon ${ }^{94}$, H. Kim, S. Luo, S. Malhotra, R. Mueller, D. Overton, D. Rathjens, A. Safonov

Texas Tech University, Lubbock, U.S.A.

N. Akchurin, J. Damgov, V. Hegde, S. Kunori, K. Lamichhane, S.W. Lee, T. Mengke, S. Muthumuni, T. Peltola, I. Volobouev, Z. Wang, A. Whitbeck

Vanderbilt University, Nashville, U.S.A.

E. Appelt, S. Greene, A. Gurrola, W. Johns, A. Melo, H. Ni, K. Padeken, F. Romeo, P. Sheldon, S. Tuo, J. Velkovska

\section{University of Virginia, Charlottesville, U.S.A.}

M.W. Arenton, B. Cox, G. Cummings, J. Hakala, R. Hirosky, M. Joyce, A. Ledovskoy, A. Li, C. Neu, B. Tannenwald, S. White, E. Wolfe

Wayne State University, Detroit, U.S.A.

N. Poudyal

University of Wisconsin - Madison, Madison, WI, U.S.A.

K. Black, T. Bose, J. Buchanan, C. Caillol, S. Dasu, I. De Bruyn, P. Everaerts, F. Fienga, C. Galloni, H. He, M. Herndon, A. Hervé, U. Hussain, A. Lanaro, A. Loeliger, R. Loveless, J. Madhusudanan Sreekala, A. Mallampalli, A. Mohammadi, D. Pinna, A. Savin, V. Shang, V. Sharma, W.H. Smith, D. Teague, S. Trembath-reichert, W. Vetens

$\dagger$ : Deceased

1 Also at TU Wien, Wien, Austria

2 Also at Institute of Basic and Applied Sciences, Faculty of Engineering, Arab Academy for Science, Technology and Maritime Transport, Alexandria, Egypt

3 Also at Université Libre de Bruxelles, Bruxelles, Belgium

4 Also at Universidade Estadual de Campinas, Campinas, Brazil 
5 Also at Federal University of Rio Grande do Sul, Porto Alegre, Brazil

6 Also at University of Chinese Academy of Sciences, Beijing, China

7 Also at Department of Physics, Tsinghua University, Beijing, China

8 Also at UFMS, Nova Andradina, Brazil

9 Also at Nanjing Normal University Department of Physics, Nanjing, China

10 Now at The University of Iowa, Iowa City, U.S.A.

11 Also at Institute for Theoretical and Experimental Physics named by A.I. Alikhanov of NRC 'Kurchatov Institute', Moscow, Russia

12 Also at Joint Institute for Nuclear Research, Dubna, Russia

13 Also at Ain Shams University, Cairo, Egypt

14 Now at British University in Egypt, Cairo, Egypt

15 Also at Purdue University, West Lafayette, U.S.A.

16 Also at Université de Haute Alsace, Mulhouse, France

17 Also at Erzincan Binali Yildirim University, Erzincan, Turkey

18 Also at CERN, European Organization for Nuclear Research, Geneva, Switzerland

19 Also at RWTH Aachen University, III. Physikalisches Institut A, Aachen, Germany

20 Also at University of Hamburg, Hamburg, Germany

21 Also at Department of Physics, Isfahan University of Technology, Isfahan, Iran

22 Also at Brandenburg University of Technology, Cottbus, Germany

23 Also at Skobeltsyn Institute of Nuclear Physics, Lomonosov Moscow State University, Moscow, Russia

24 Also at Physics Department, Faculty of Science, Assiut University, Assiut, Egypt

25 Also at Karoly Robert Campus, MATE Institute of Technology, Gyongyos, Hungary

26 Also at Institute of Physics, University of Debrecen, Debrecen, Hungary

27 Also at Institute of Nuclear Research ATOMKI, Debrecen, Hungary

28 Also at MTA-ELTE Lendület CMS Particle and Nuclear Physics Group, Eötvös Loránd University, Budapest, Hungary

29 Also at Wigner Research Centre for Physics, Budapest, Hungary

30 Also at IIT Bhubaneswar, Bhubaneswar, India

31 Also at Institute of Physics, Bhubaneswar, India

32 Also at G.H.G. Khalsa College, Punjab, India

33 Also at Shoolini University, Solan, India

34 Also at University of Hyderabad, Hyderabad, India

35 Also at University of Visva-Bharati, Santiniketan, India

36 Also at Indian Institute of Technology (IIT), Mumbai, India

37 Also at Deutsches Elektronen-Synchrotron, Hamburg, Germany

38 Also at Sharif University of Technology, Tehran, Iran

39 Also at Department of Physics, University of Science and Technology of Mazandaran, Behshahr, Iran

40 Now at INFN Sezione di Bari ${ }^{a}$, Università di Bari ${ }^{b}$, Politecnico di Bari ${ }^{c}$, Bari, Italy

41 Also at Italian National Agency for New Technologies, Energy and Sustainable Economic Development, Bologna, Italy

42 Also at Centro Siciliano di Fisica Nucleare e di Struttura Della Materia, Catania, Italy

43 Also at Università di Napoli 'Federico II', Napoli, Italy

44 Also at Consiglio Nazionale delle Ricerche - Istituto Officina dei Materiali, PERUGIA, Italy

45 Also at Riga Technical University, Riga, Latvia

46 Also at Consejo Nacional de Ciencia y Tecnología, Mexico City, Mexico

47 Also at IRFU, CEA, Université Paris-Saclay, Gif-sur-Yvette, France 
48 Also at Institute for Nuclear Research, Moscow, Russia

49 Now at National Research Nuclear University 'Moscow Engineering Physics Institute' (MEPhI), Moscow, Russia

50 Also at Institute of Nuclear Physics of the Uzbekistan Academy of Sciences, Tashkent, Uzbekistan

51 Also at St. Petersburg State Polytechnical University, St. Petersburg, Russia

52 Also at University of Florida, Gainesville, U.S.A.

53 Also at Imperial College, London, United Kingdom

54 Also at P.N. Lebedev Physical Institute, Moscow, Russia

55 Also at Moscow Institute of Physics and Technology, Moscow, Russia, Moscow, Russia

56 Also at California Institute of Technology, Pasadena, U.S.A.

57 Also at Budker Institute of Nuclear Physics, Novosibirsk, Russia

58 Also at Faculty of Physics, University of Belgrade, Belgrade, Serbia

59 Also at Trincomalee Campus, Eastern University, Sri Lanka, Nilaveli, Sri Lanka

60 Also at INFN Sezione di Pavia ${ }^{a}$, Università di Pavia ${ }^{b}$, Pavia, Italy

61 Also at National and Kapodistrian University of Athens, Athens, Greece

62 Also at Ecole Polytechnique Fédérale Lausanne, Lausanne, Switzerland

63 Also at Universität Zürich, Zurich, Switzerland

64 Also at Stefan Meyer Institute for Subatomic Physics, Vienna, Austria

65 Also at Laboratoire d'Annecy-le-Vieux de Physique des Particules, IN2P3-CNRS, Annecyle-Vieux, France

66 Also at Sirnak University, Sirnak, Turkey

67 Also at Near East University, Research Center of Experimental Health Science, Nicosia, Turkey

68 Also at Konya Technical University, Konya, Turkey

69 Also at Istanbul University - Cerrahpasa, Faculty of Engineering, Istanbul, Turkey

70 Also at Piri Reis University, Istanbul, Turkey

71 Also at Adiyaman University, Adiyaman, Turkey

72 Also at Ozyegin University, Istanbul, Turkey

73 Also at Izmir Institute of Technology, Izmir, Turkey

74 Also at Necmettin Erbakan University, Konya, Turkey

75 Also at Bozok Universitetesi Rektörlügü, Yozgat, Turkey

76 Also at Marmara University, Istanbul, Turkey

77 Also at Milli Savunma University, Istanbul, Turkey

78 Also at Kafkas University, Kars, Turkey

79 Also at Istanbul Bilgi University, Istanbul, Turkey

80 Also at Hacettepe University, Ankara, Turkey

81 Also at Rutherford Appleton Laboratory, Didcot, United Kingdom

82 Also at Vrije Universiteit Brussel, Brussel, Belgium

83 Also at School of Physics and Astronomy, University of Southampton, Southampton, United Kingdom

84 Also at IPPP Durham University, Durham, United Kingdom

85 Also at Monash University, Faculty of Science, Clayton, Australia

86 Also at Università di Torino, TORINO, Italy

87 Also at Bethel University, St. Paul, Minneapolis, U.S.A., St. Paul, U.S.A.

88 Also at Karamanoğlu Mehmetbey University, Karaman, Turkey

89 Also at Bingol University, Bingol, Turkey

90 Also at Georgian Technical University, Tbilisi, Georgia 
91 Also at Sinop University, Sinop, Turkey

92 Also at Erciyes University, KAYSERI, Turkey

93 Also at Texas A\&M University at Qatar, Doha, Qatar

94 Also at Kyungpook National University, Daegu, Korea, Daegu, Korea 